\title{
Zirconium Complexes Supported by a Ferrocene-Based Ligand as Redox Switches for Hydroamination Reactions
}

Yi Shen, Scott M. Shepard, Christopher J. Reed, and Paula L. Diaconescu*

Department of Chemistry and Biochemistry, University of California, Los Angeles, CA 90095

\section{Table of Contents}

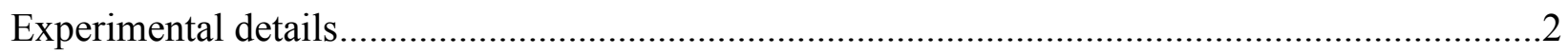

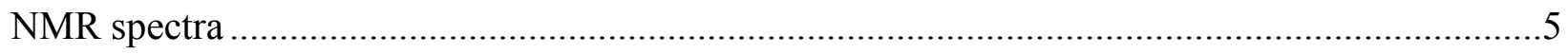

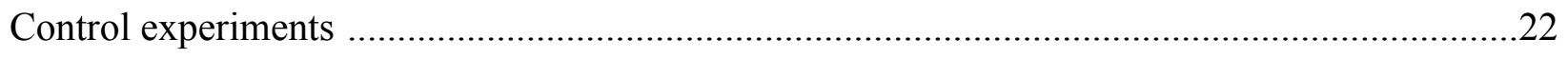

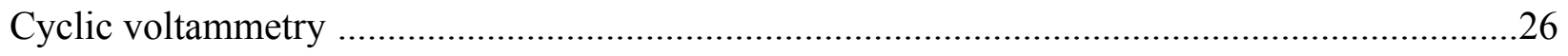

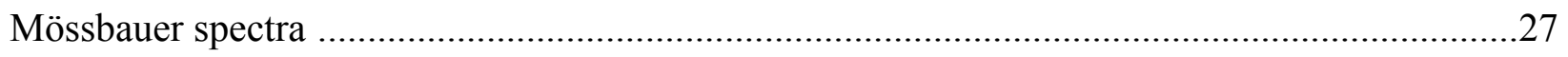

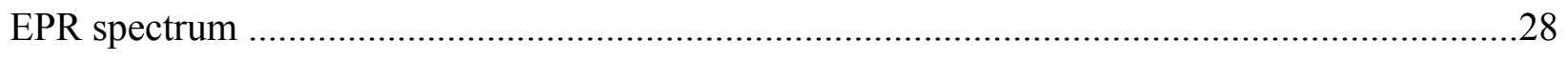

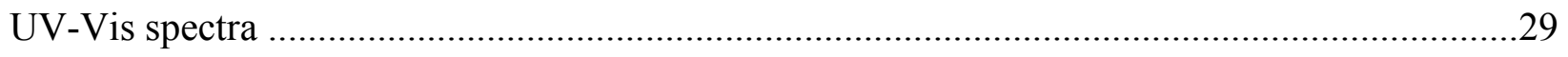

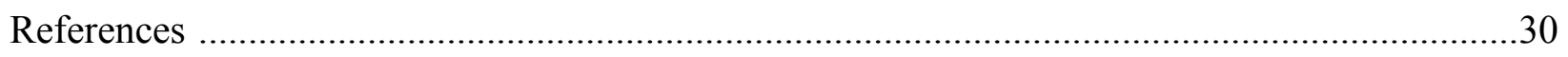


General considerations. All reactions were performed using standard Schlenk techniques or in an MBraun drybox under a nitrogen atmosphere, unless otherwise noted. All glassware and Celite were stored in an oven at $100{ }^{\circ} \mathrm{C}$ overnight before being brought into the drybox. Solvents, except for $n$-pentane, were purified by the method of Grubbs ${ }^{1}$ and transferred to the glovebox without exposure to air. $n$-Pentane was purified by stirring over calcium hydride overnight followed by a distillation under a nitrogen atmosphere and transferred into the glovebox without exposure to air. NMR solvents were obtained from Cambridge Isotope Laboratories, degassed, and stored over activated molecular sieves prior to use. NMR spectra were recorded at ambient temperature on Bruker AV-300, AV-400, AV-500, and DRX-500 spectrometers. Chemical shifts are reported with respect to internal solvent, $7.16 \mathrm{ppm}\left(\mathrm{C}_{6} \mathrm{D}_{6}\right)$ and $7.26 \mathrm{ppm}\left(\mathrm{CDCl}_{3}\right)$ for ${ }^{1} \mathrm{H}$ NMR spectra. All reagents were acquired from commercial sources and used as received unless otherwise noted. $\mathrm{Zr}\left(\mathrm{NEt}_{2}\right)_{4},{ }^{2} \mathrm{NaBAr}^{\mathrm{F}},{ }^{3}$ and $\mathrm{H}_{2}$ (thiolfan* ${ }^{4}{ }^{4}$ were synthesized according to published procedures. Amine substrates were synthesized according to published procedures. ${ }^{5-11}$ Cyclic voltammograms were acquired with a $\mathrm{CH}$ Instruments $\mathrm{CHI} 630 \mathrm{D}$ potentiostat and recorded with $\mathrm{CH}$ Instruments software (version 13.04). All potentials are given with respect to the ferrocene-ferrocenium couple. Elemental analyses were performed on an Exeter Analytical Inc. CE-440 elemental analyzer. HRMS was recorded on an Agilent 7250 Accurate-mass Q-TOF GC/MS (ESI Source). UV-Vis spectra were recorded on a Hewlett Packard 8453. Zero applied field ${ }^{57} \mathrm{Fe}$ Mössbauer spectra were recorded at $80 \mathrm{~K}$ in constant acceleration mode on a spectrometer from See Co (Edina, MN) equipped with an SVT-400 cryostat (Janis, Wilmington, WA). The isomer shifts are relative to the centroid of an $\alpha-\mathrm{Fe}$ foil signal at room temperature. Samples were prepared by mixing polycrystalline material (30-40 mg) with boron nitride in a cup fitted with a screw cap. The data were fit to Lorentzian lineshapes using WMOSS (www.wmoss.org). X-band CW EPR spectra were acquired on a Bruker (Billerica, MA) EMX spectrometer using Bruker Win-EPR software (ver. 3.0). Temperature control was achieved using liquid helium and an Oxford Instruments (Oxford, UK) ESR-900 cryogen flow cryostat and an ITC-503 temperature controller.

\section{Synthesis of (thiolfan*) $\mathrm{Zr}\left(\mathrm{NEt}_{2}\right)_{2}$}

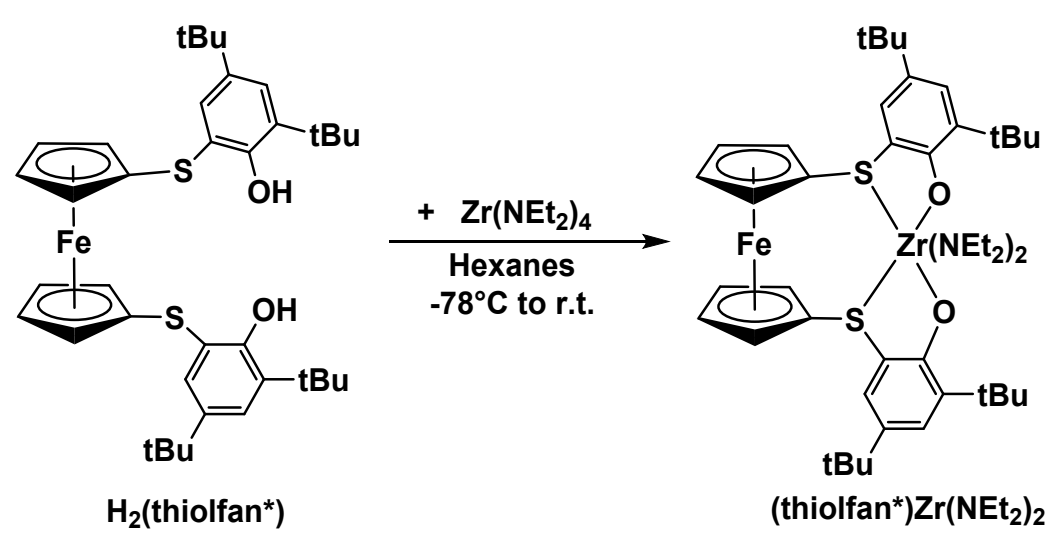

$0.201 \mathrm{~g}(0.306 \mathrm{mmol})$ of $\mathrm{H}_{2}$ (thiolfan*) was dissolved in $10 \mathrm{~mL}$ of hexanes and cooled to $-78^{\circ} \mathrm{C}$ in a $25 \mathrm{~mL}$ vial. $0.101 \mathrm{~g}(0.306 \mathrm{mmol})$ of $\mathrm{Zr}\left(\mathrm{NEt}_{2}\right)_{4}$ was dissolved in $2 \mathrm{~mL}$ of hexanes in a separate vial and cooled to $-78^{\circ} \mathrm{C} . \mathrm{H}_{2}$ (thiolfan*) solution was added dropwise to $\mathrm{Zr}\left(\mathrm{NEt}_{2}\right)_{4}$ and the reaction solution was stirred at room temperature for $30 \mathrm{~min}$. The volatiles were removed under a reduced pressure. The crude yellow solid was recrystallized from pentanes giving $0.2 \mathrm{~g}(77 \%)$ of clean 
(thiolfan*) Zr( $\left.\mathrm{NEt}_{2}\right)_{2} .{ }^{1} \mathrm{H}$ NMR $\left(\mathrm{C}_{6} \mathrm{D}_{6}, 300 \mathrm{MHz}, 25^{\circ} \mathrm{C}\right): \delta 1.07\left(\mathrm{t}, 12 \mathrm{H}, \mathrm{NCH}_{2} \mathrm{CH}_{3}\right), 1.24(\mathrm{~s}, 18 \mathrm{H}$, $\left.\mathrm{C}\left(\mathrm{CH}_{3}\right)_{3}\right), 1.76\left(\mathrm{~s}, 18 \mathrm{H}, \mathrm{C}\left(\mathrm{CH}_{3}\right)_{3}\right), 3.68\left(\mathrm{~s}, 6 \mathrm{H}, \mathrm{NCH}_{2} \mathrm{CH}_{3}\right), 3.90$ (br, $10 \mathrm{H}, \mathrm{NCH}_{2} \mathrm{CH}_{3}$ and $\mathrm{Cp}-$ $H), 7.57(\mathrm{~d}, 2 \mathrm{H}, \mathrm{ArH}), 7.60(\mathrm{~d}, 2 \mathrm{H}, \mathrm{ArH}) .{ }^{13} \mathrm{C}$ NMR $\left(\mathrm{C}_{6} \mathrm{D}_{6}, 500 \mathrm{MHz}, 25^{\circ} \mathrm{C}\right): \delta 14.79\left(\mathrm{NCH}_{2} \mathrm{CH}_{3}\right)$, $\left.29.6\left(\mathrm{C}_{(\mathrm{CH}}\right)_{3}\right), 31.3\left(\mathrm{C}\left(\mathrm{CH}_{3}\right)_{3}\right), 34.0\left(\mathrm{C}\left(\mathrm{CH}_{3}\right)_{3}\right), 35.4\left(\mathrm{C}\left(\mathrm{CH}_{3}\right)_{3}\right), 42.5\left(\mathrm{NCH}_{2} \mathrm{CH}_{3}\right), 65.1$, 68.8,.70.5, 89.97 (Cp), 120.2 (ArC), 125.6 (ArCH), 130.6 (ArCH), 137.6, $(\operatorname{ArC}), 140.3(\operatorname{ArC})$, 163.0 (ArC). Anal. Calcd for (thiolfan*) $\mathrm{Zr}\left(\mathrm{NEt}_{2}\right)_{2}\left(\mathrm{C}_{46} \mathrm{H}_{68} \mathrm{FeN}_{2} \mathrm{O}_{2} \mathrm{~S} 2 \mathrm{Zr}\right)$ : C, 61.92\%; H, 7.68\%; N, $3.14 \%$. Found: C, $62.28 \%$; H, $7.44 \%$; N, $2.50 \%$.

Synthesis of [(thiolfan*) Zr( $\left.\left.\mathbf{N E t}_{2}\right)_{2}\right]\left[\mathbf{B A r}^{\mathrm{F}}\right]: 0.183 \mathrm{~g}(0.2055 \mathrm{mmol})$ of (thiolfan*) $\mathrm{Zr}\left(\mathrm{NEt}_{2}\right)_{2}$ was dissolved in $1 \mathrm{~mL}$ of $o$-difluorobenzene and added to a $2 \mathrm{~mL} o$-difluorobenzene solution of $\left[{ }^{\mathrm{Ac}} \mathrm{Fc}\right]\left[\mathrm{BAr}{ }^{\mathrm{F}}\right](0.201 \mathrm{mg}, 0.9 \mathrm{eq}, 0.1850 \mathrm{mmol})$ and stirred for 1 hour at room temperature. The volatiles were removed under a reduced pressure. The oily product was washed three times with $25 \mathrm{~mL}$ of hexanes each time. $2 \mathrm{~mL}$ of toluene was added in the last wash to remove acetylferrocene. The resulting solid was dissolved in benzene, the solution concentrated and layered with hexanes at room temperature overnight. The product was then dried under a reduced pressure for $24 \mathrm{~h}$ yielding $0.105 \mathrm{mg}(32 \%)$ of a dark purple solid. Anal. Calcd for [(thiolfan*) $\left.\mathrm{Zr}\left(\mathrm{NEt}_{2}\right)_{2}\right]\left[\mathrm{BAr}^{\mathrm{F}}\right.$ ] $\left(\mathrm{C}_{78} \mathrm{H}_{80} \mathrm{FeN}_{2} \mathrm{O}_{2} \mathrm{~S}_{2} \mathrm{BF}_{24} \mathrm{Zr}\right): \mathrm{C}, 53.37 \%$; H, $4.59 \%$; N, 1.59\%. Found: $\mathrm{C}, 52.71 \%$; H, 4.16\%; N, $1.17 \%$.

Synthetic procedure for ${ }^{\mathrm{Ac}} \mathbf{F c B A r}{ }^{\mathrm{F}}$ (slightly modified from previously reported procedures): ${ }^{12,13}$ $209.3 \mathrm{mg}(0.665 \mathrm{mmol})$ of ${ }^{\mathrm{Ac}} \mathrm{FcBF}_{4}$ was dissolved in $\mathrm{CH}_{2} \mathrm{Cl}_{2}$ and added to $590.7 \mathrm{mg}(0.665 \mathrm{mmol})$ of $\mathrm{NaBAr}^{\mathrm{F}}$ in $\mathrm{CH}_{2} \mathrm{Cl}_{2}$. The reaction mixture was stirred for 2 hours at room temperature and the volatiles were removed under a reduced pressure. The solid was extracted with diethyl ether and the resulting solution was filtered through Celite. ${ }^{\mathrm{Ac}} \mathrm{FcBAr}^{\mathrm{F}}$ was crystallized from concentrated diethyl ether layered with hexanes at $-33{ }^{\circ} \mathrm{C}$ yielding dark blue crystals; $611 \mathrm{mg}(84 \%)$.

\section{Synthesis of 12a:}

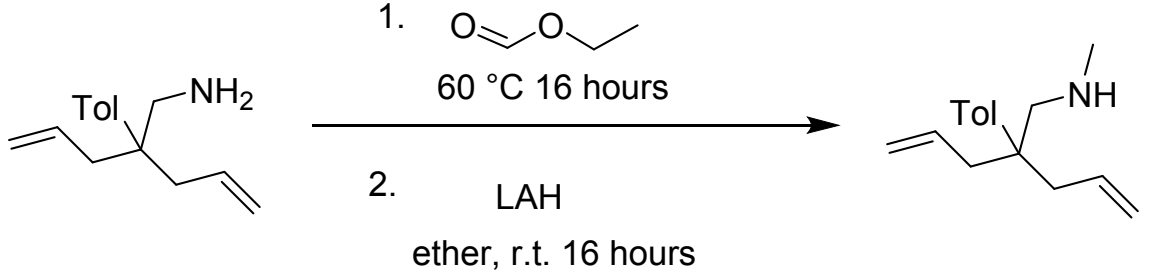

$0.188 \mathrm{~g}(0.873 \mathrm{mmol})$ of 2-allyl-2-(p-tolyl)pent-4-en-amine was dissolved in $1 \mathrm{~mL}$ of ethyl formate in a Schlenk flask under an inert atmosphere. The reaction mixture was stirred at $60{ }^{\circ} \mathrm{C}$ for $16 \mathrm{~h}$. Ethyl formate was removed under a reduced pressure, and the residual was dissolved in diethyl ether, and added to a diethylether solution of $\mathrm{LiAlH}_{4}(99 \mathrm{mg}, 2.619 \mathrm{mmol})$ then stirred for $16 \mathrm{~h}$. The reaction was quenched by a $2 \mathrm{M} \mathrm{NaOH}$ solution at $0^{\circ} \mathrm{C}$. The product was extracted into diethyl ether and dried over $\mathrm{MgSO}_{4}$. The volatiles were removed under a reduced pressure yielding a light yellow gel as the product, which was used without a further purification. ${ }^{1} \mathrm{H}$ NMR spectrum $\left(\mathrm{CDCl}_{3}, 500 \mathrm{MHz}, 298 \mathrm{~K}\right.$ ) of 12a: $\delta 2.30\left(\mathrm{~s}, 3 \mathrm{H}, \mathrm{NHCH}_{3}\right), 2.33$ (s, 3H, $\left.\mathrm{PhCH}_{3}\right), 2.51$ (ddd, $4 \mathrm{H}$, $\left.\mathrm{CH}_{2}=\mathrm{CHCH}_{2}\right), 5.04\left(\mathrm{~m}, 4 \mathrm{H}, \mathrm{CH}_{2}=\mathrm{CH}\right), 5.65\left(\mathrm{~m}, 2 \mathrm{H}, \mathrm{CH}_{2}=\mathrm{CH}\right), 7.13(\mathrm{~d}, 2 \mathrm{H}, \mathrm{Ar} H), 7.23(\mathrm{~d}, 2 \mathrm{H}$, $\operatorname{Ar} H$ ). HRMS (ESI) calculated for $\mathrm{C}_{16} \mathrm{H}_{24} \mathrm{~N}^{+}[\mathrm{M}+\mathrm{H}]^{+} 230.1909$, found 230.2273 . 
General procedure for hydroamination reactions catalyzed by (thiolfan*) $\operatorname{Zr}\left(\mathrm{NEt}_{2}\right)_{2}$ : (thiolfan*) $\mathrm{Zr}\left(\mathrm{NEt}_{2}\right)_{2}\left(0.01 \mathrm{mmol}, 10 \mathrm{~mol} \%\right.$ ) was dissolved in $\mathrm{C}_{6} \mathrm{D}_{6}$ and added to a J-Young NMR tube. The substrate $(0.1 \mathrm{mmol})$ was also dissolved in $\mathrm{C}_{6} \mathrm{D}_{6}$ and added to the J-Young tube. The tube was sealed and analyzed for a 0 -hour ${ }^{1} \mathrm{H}$ NMR spectrum before being heated to $100{ }^{\circ} \mathrm{C}$. Reactions were monitored by ${ }^{1} \mathrm{H}$ NMR spectroscopy.

General procedure for hydroamination reactions catalyzed by the oxidized state of (thiolfan*) Zr( $\left.\mathbf{N E t}_{2}\right)_{2}$ : (thiolfan*) $\mathrm{Zr}\left(\mathrm{NEt}_{2}\right)_{2}(0.01 \mathrm{mmol}, 10 \mathrm{~mol} \%)$ was dissolved in $\mathrm{C}_{6} \mathrm{D}_{6}$ and added to a J-Young NMR tube. ${ }^{\mathrm{Ac}} \mathrm{FcBAr}^{\mathrm{F}}$ (10.91 mg, $0.009 \mathrm{mmol}, 9 \mathrm{~mol} \%$ ) was then added to the J-Young tube leading to a color change from yellow to brown. The tube was shaken until all solids dissolved. Then the substrate $(0.1 \mathrm{mmol})$ was dissolved in $\mathrm{C}_{6} \mathrm{D}_{6}$ and added to the J-Young tube. The tube was then sealed and analyzed for a 0 -hour ${ }^{1} \mathrm{H}$ NMR spectrum before being heated to $100^{\circ} \mathrm{C}$. Reactions were monitored by ${ }^{1} \mathrm{H}$ NMR spectroscopy.

General procedure for control experiments: ${ }^{\mathrm{Ac}} \mathrm{FcBAr}^{\mathrm{F}}(0.01 \mathrm{mmol}, 10 \mathrm{~mol} \%)$ was dissolved in $\mathrm{C}_{6} \mathrm{D}_{6}$ and added to a J-Young NMR tube. The substrate $(0.1 \mathrm{mmol})$ was dissolved in $\mathrm{C}_{6} \mathrm{D}_{6}$ and added to the J-Young tube. The tube was then sealed and analyzed for a 0 -hour ${ }^{1} \mathrm{H}$ NMR spectrum before being heated to $100{ }^{\circ} \mathrm{C}$. Reactions were monitored by ${ }^{1} \mathrm{H}$ NMR spectroscopy. 
NMR spectra

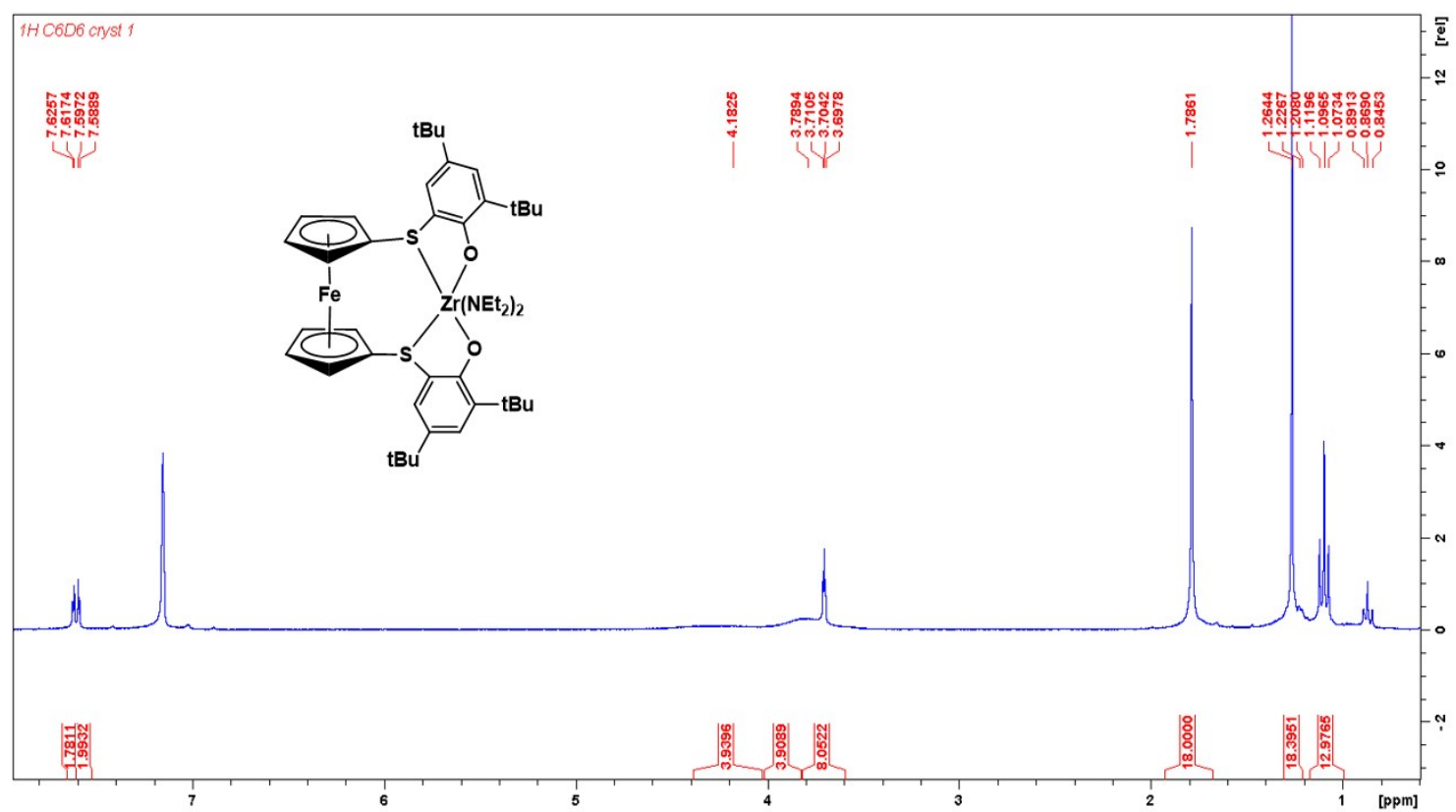

Figure S1: ${ }^{1} \mathrm{H}$ NMR spectrum $\left(\mathrm{C}_{6} \mathrm{D}_{6}, 500 \mathrm{MHz}, 298 \mathrm{~K}\right)$ of (thiolfan*) $\mathrm{Zr}\left(\mathrm{NEt}_{2}\right)_{2}: \delta 1.07(\mathrm{t}, 12 \mathrm{H}$, $\left.\mathrm{NCH}_{2} \mathrm{CH}_{3}\right), 1.26\left(\mathrm{~s}, 18 \mathrm{H}, \mathrm{C}\left(\mathrm{CH}_{3}\right)_{3}\right), 1.78\left(\mathrm{~s}, 18 \mathrm{H}, \mathrm{C}\left(\mathrm{CH}_{3}\right)_{3}\right), 3.71\left(\mathrm{~s}, 8 \mathrm{H}, \mathrm{NCH}_{2} \mathrm{CH}_{3}\right), 3.78$ (br, $4 \mathrm{H}$, $\mathrm{Cp}-H), 4.10(\mathrm{br}, 4 \mathrm{H}, \mathrm{Cp}-H), 7.58(\mathrm{~d}, 2 \mathrm{H}, \operatorname{Ar} H), 7.60(\mathrm{~d}, 2 \mathrm{H}, \mathrm{Ar} H)$. The peak at $0.89 \mathrm{ppm}$ is attributed to residual $n$-pentane.

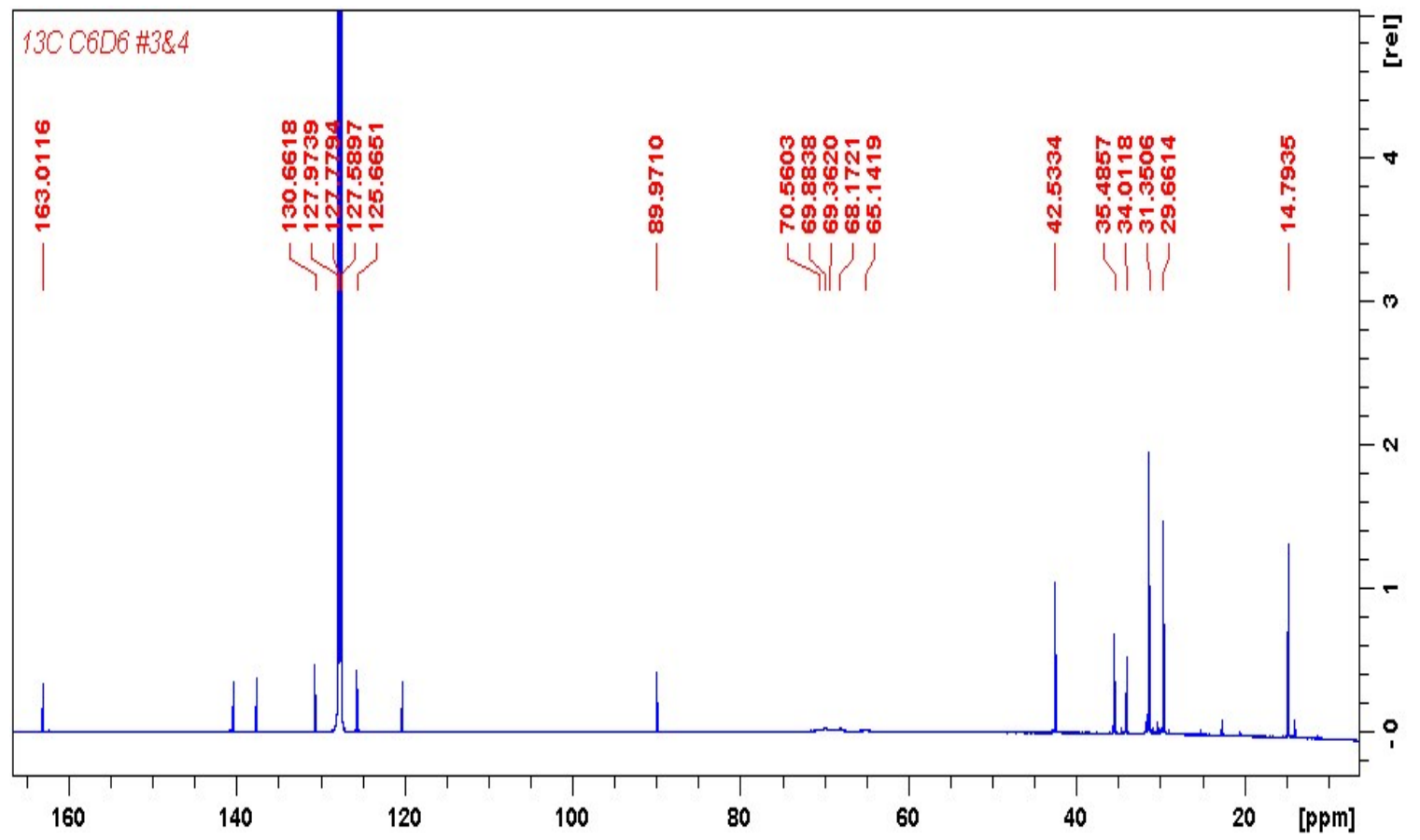

Figure S2: ${ }^{13} \mathrm{C}$ NMR spectrum $\left(\mathrm{C}_{6} \mathrm{D}_{6}, 500 \mathrm{MHz}, 298 \mathrm{~K}\right)$ of (thiolfan*) $\mathrm{Zr}\left(\mathrm{NEt}_{2}\right)_{2}: \delta 14.79$

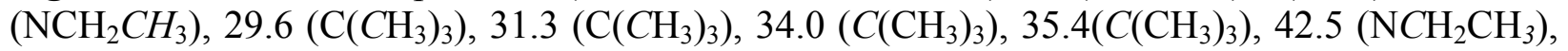
65.1, 68.8,.70.5, $89.97(\mathrm{Cp}), 120.2(\mathrm{ArC}), 125.6(\mathrm{ArCH}), 130.6(\mathrm{ArCH}), 137.6,(\mathrm{ArC}), 140.3$ $(\mathrm{ArC}), 163.0(\mathrm{ArC})$. 


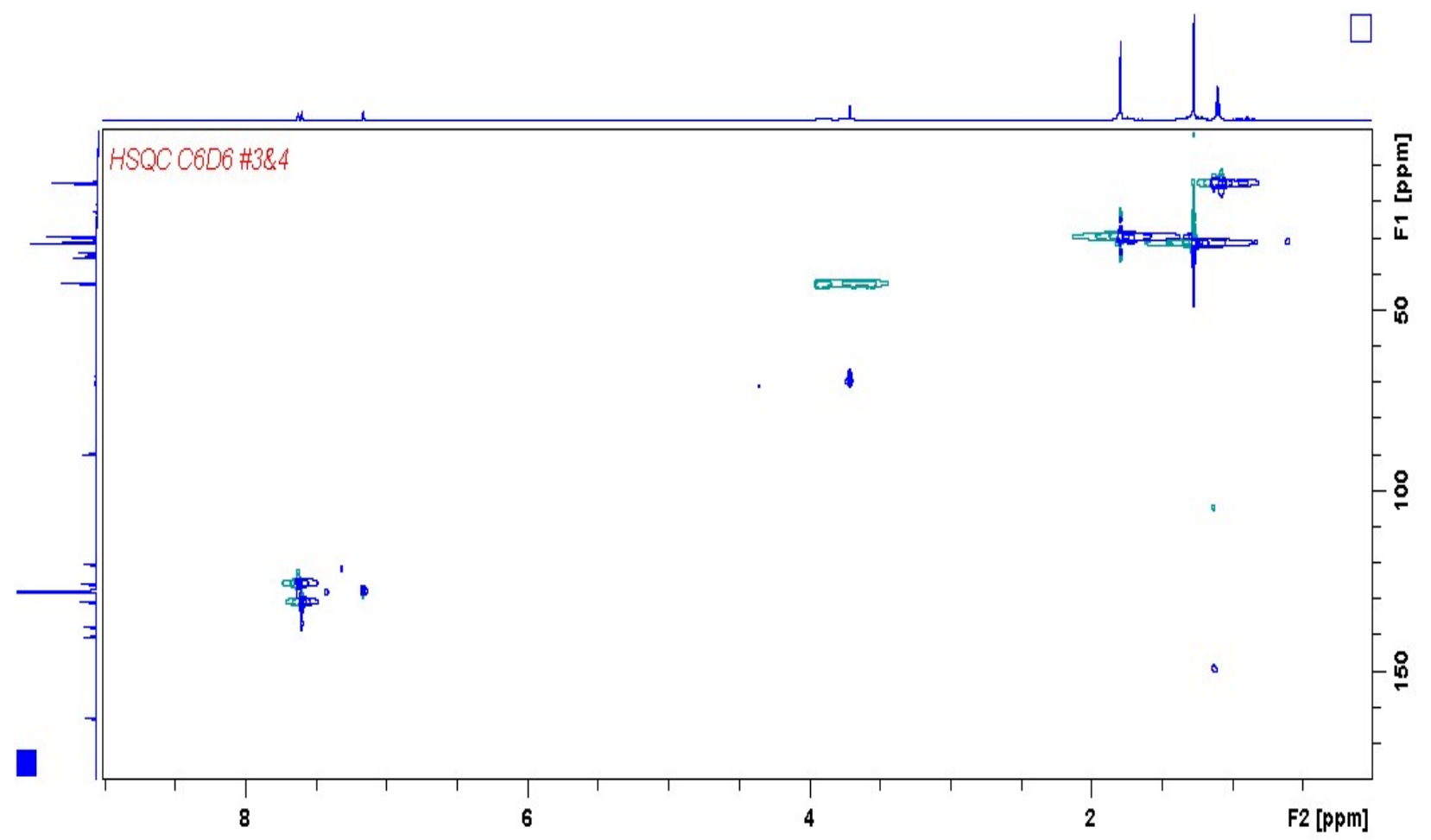

Figure S3: HSQC spectrum $\left(\mathrm{C}_{6} \mathrm{D}_{6}, 500 \mathrm{MHz}, 298 \mathrm{~K}\right)$ of (thiolfan*) $\mathrm{Zr}\left(\mathrm{NEt}_{2}\right)_{2}$.

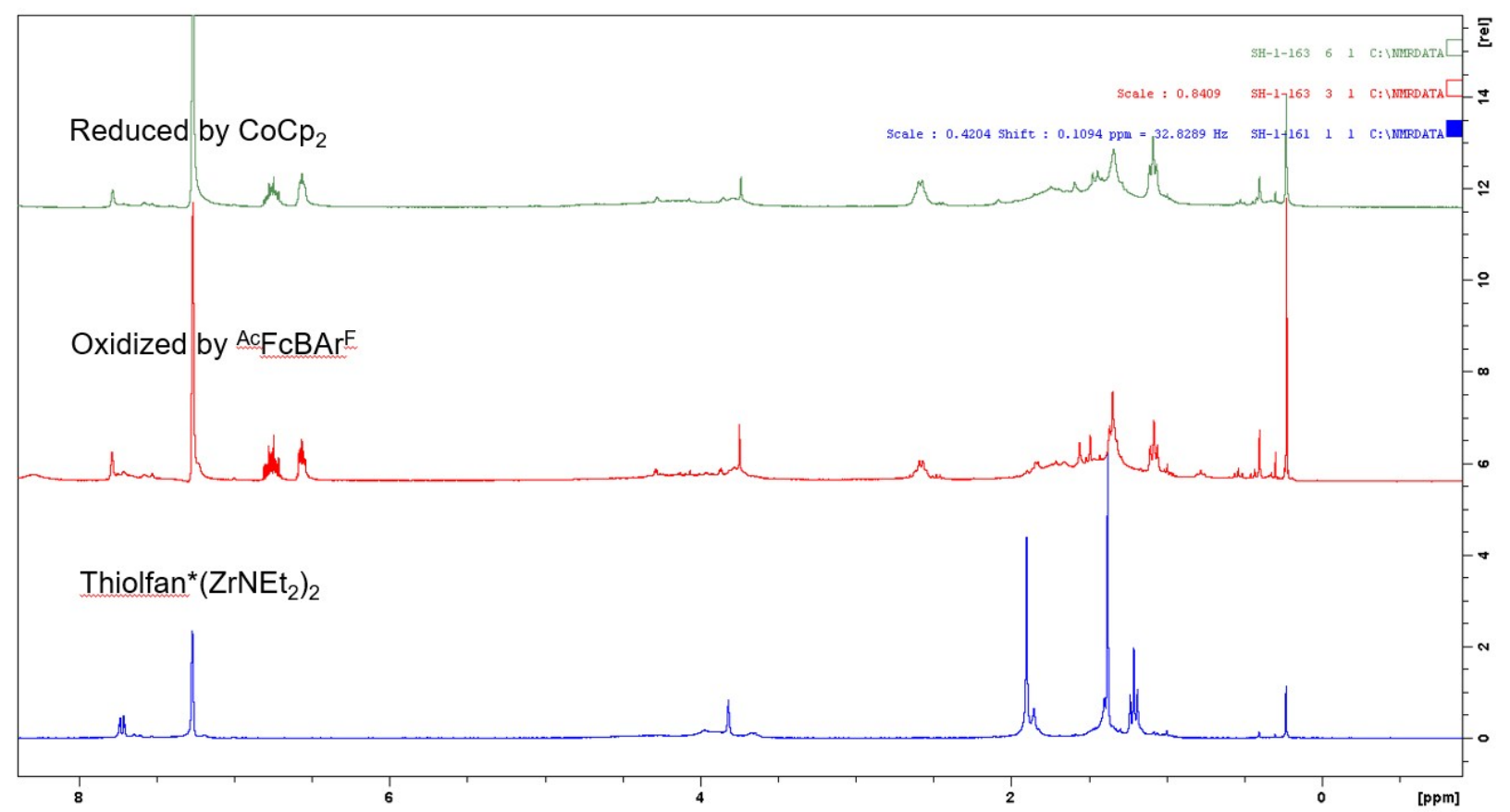

Figure S4: ${ }^{1} \mathrm{H}$ NMR spectrum $\left(\mathrm{C}_{6} \mathrm{D}_{6}, 300 \mathrm{MHz}, 298 \mathrm{~K}\right)$ of (thiolfan*) $\mathrm{Zr}\left(\mathrm{NEt}_{2}\right)_{2}$ chemical oxidation and reduction. 


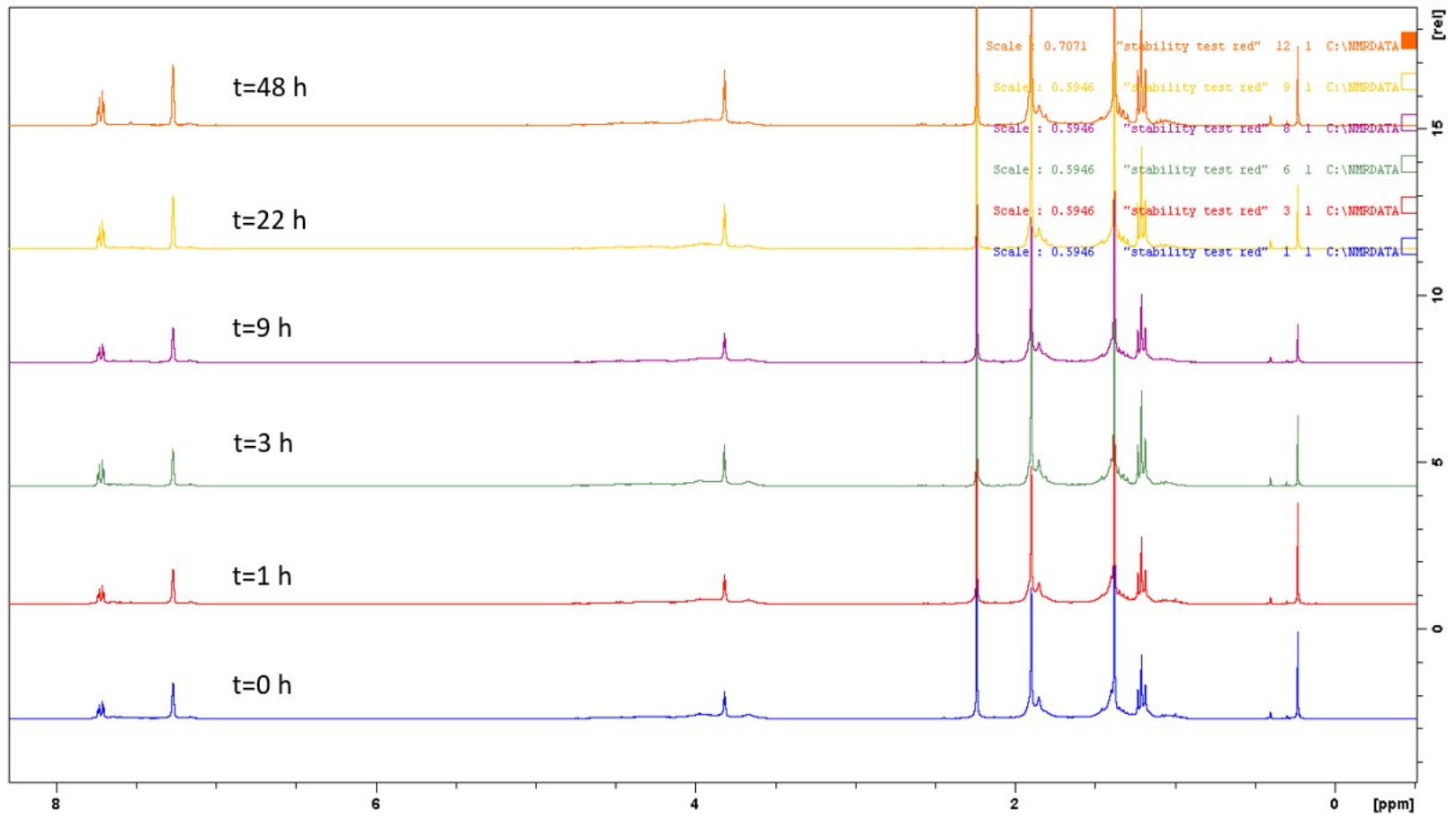

Figure S5: ${ }^{1} \mathrm{H}$ NMR spectrum $\left(\mathrm{C}_{6} \mathrm{D}_{6}, 300 \mathrm{MHz}, 298 \mathrm{~K}\right)$ of (thiolfan*) $\mathrm{Zr}\left(\mathrm{NEt}_{2}\right)_{2}$ stability test in $\mathrm{C}_{6} \mathrm{D}_{6}$ at $100{ }^{\circ} \mathrm{C}$ for 48 hours.

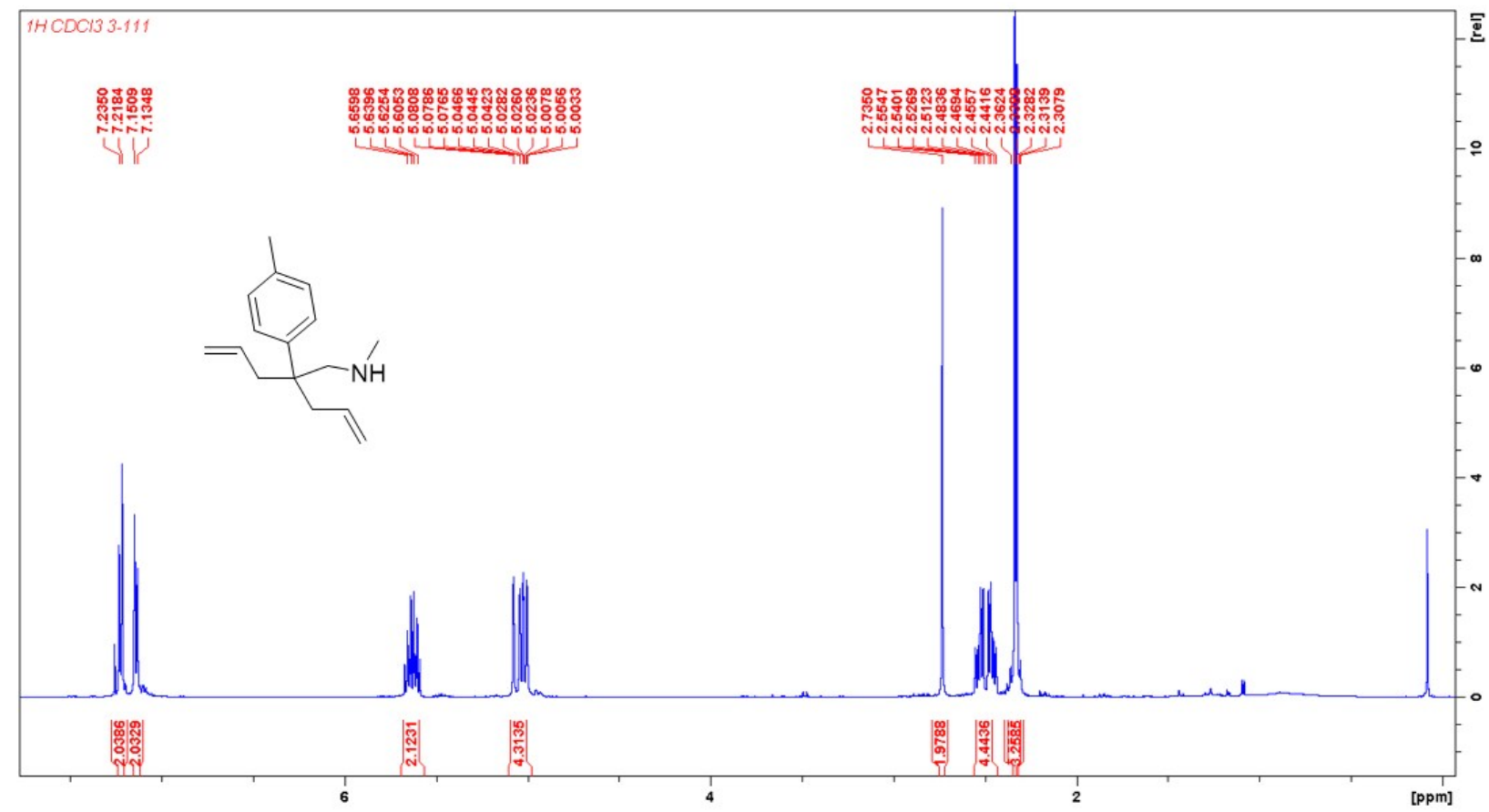

Figure S6: ${ }^{1} \mathrm{H}$ NMR spectrum $\left(\mathrm{CDCl}_{3}, 500 \mathrm{MHz}, 298 \mathrm{~K}\right)$ of 12a: $\delta 2.30\left(\mathrm{~s}, 3 \mathrm{H}, \mathrm{NHCH}_{3}\right), 2.33(\mathrm{~s}$, $3 \mathrm{H}, \mathrm{PhCH}_{3}$ ), 2.51 (ddd, 4H, $\left.\mathrm{CH}_{2}=\mathrm{CHCH}_{2}\right), 2.73$ (s, 2H, $\left.\mathrm{NHCH}_{2}\right), 5.04\left(\mathrm{~m}, 4 \mathrm{H}, \mathrm{CH}_{2}=\mathrm{CH}\right), 5.65$ $\left(\mathrm{m}, 2 \mathrm{H}, \mathrm{CH}_{2}=\mathrm{CH}\right), 7.13(\mathrm{~d}, 2 \mathrm{H}, \mathrm{ArH}), 7.23(\mathrm{~d}, 2 \mathrm{H}, \mathrm{ArH})$. 


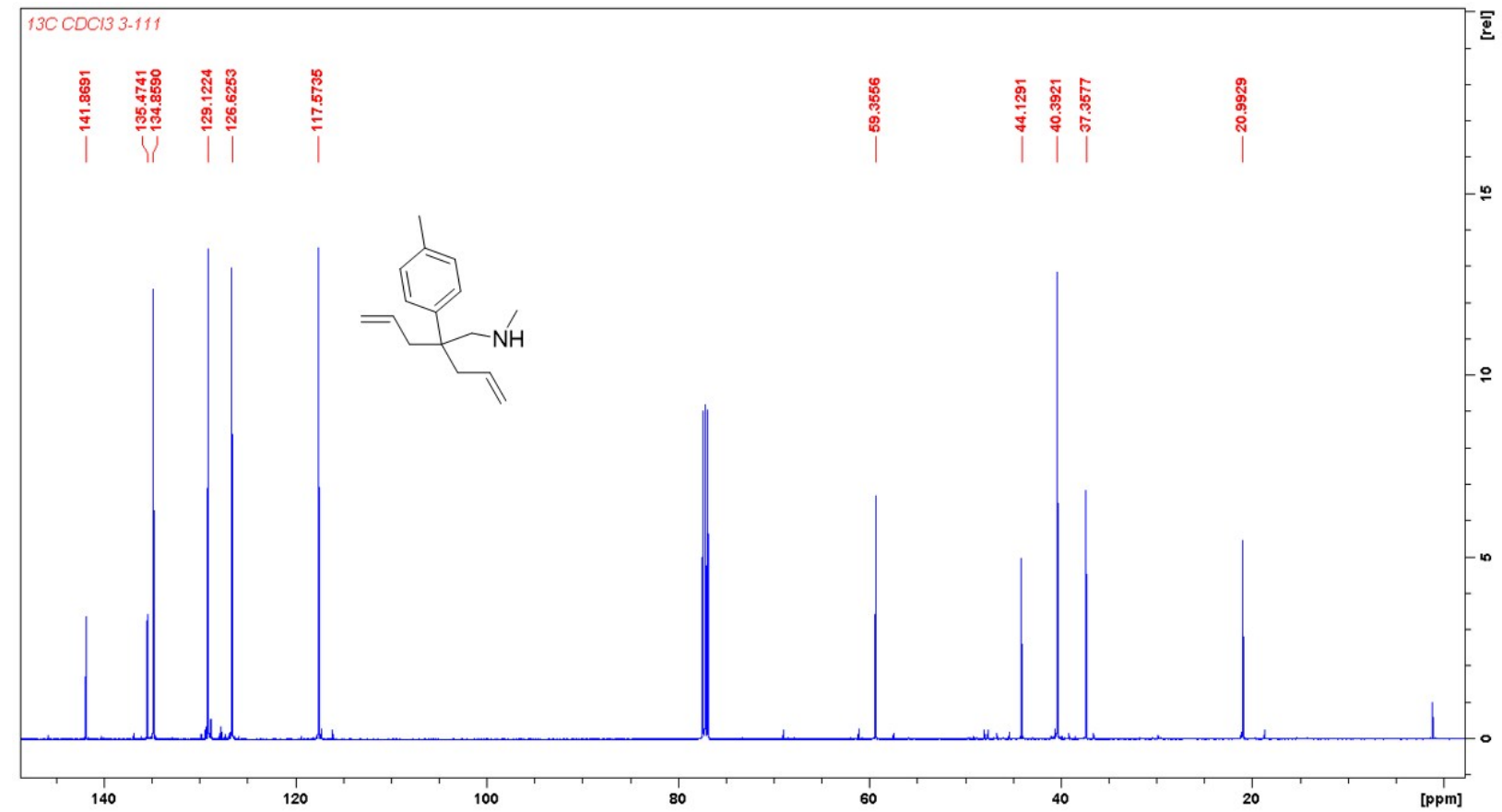

Figure S7: ${ }^{13} \mathrm{C}$ NMR spectrum $\left(\mathrm{CDCl}_{3}, 500 \mathrm{MHz}, 298 \mathrm{~K}\right)$ of 12a: $\delta 20.99\left(\mathrm{PhCH}_{3}\right), 37.35$ $\left(\mathrm{NHCH}_{3}\right), 40.39\left(\mathrm{CH}_{2}=\mathrm{CHCH}_{2}\right), 59.35\left(\mathrm{NHCH}_{2}\right), 117.57\left(\mathrm{CH}_{2}=\mathrm{CH}\right), 126.62(\mathrm{ArCH}), 129.12$ $(\mathrm{ArCH}), 134.85\left(\mathrm{CH}_{2}=\mathrm{CH}\right)$.

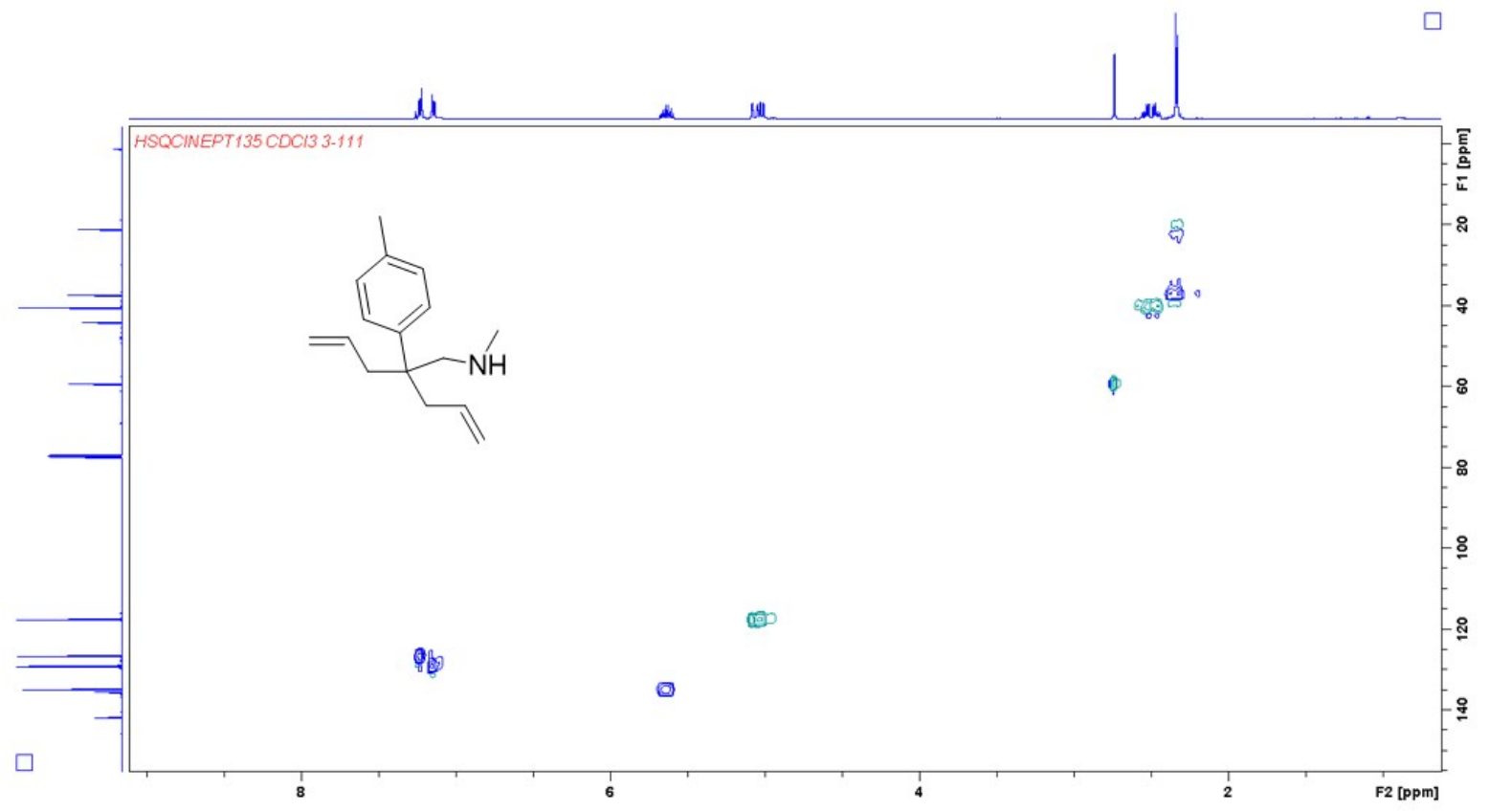

Figure S8: HSQC spectrum $\left(\mathrm{CDCl}_{3}, 500 \mathrm{MHz}, 298 \mathrm{~K}\right)$ of 12a. 


\section{Hydroamination reactions:}

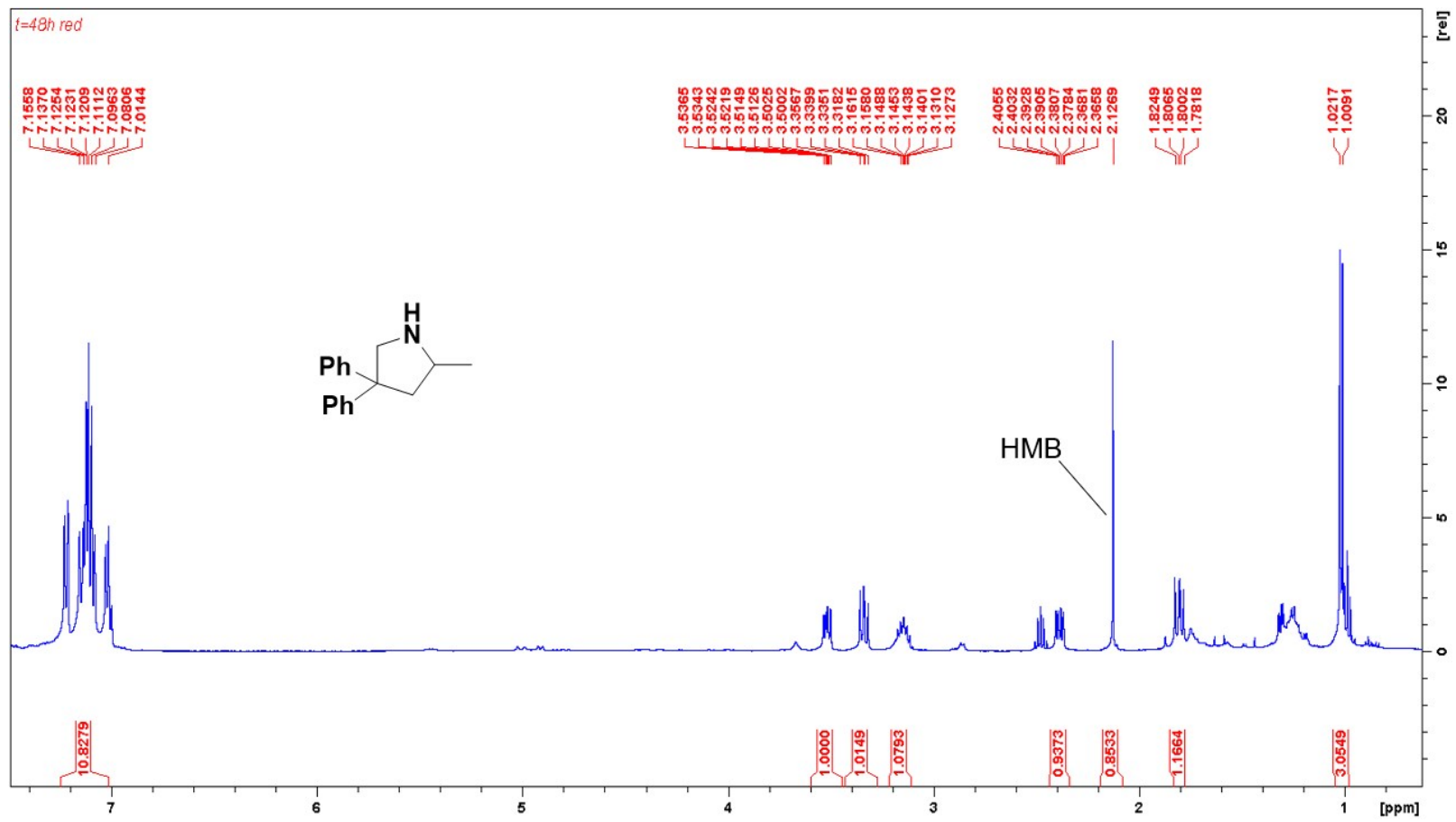

Figure S9: ${ }^{1} \mathrm{H}$ NMR spectrum $\left(\mathrm{C}_{6} \mathrm{D}_{6}, 300 \mathrm{MHz}, 298 \mathrm{~K}\right)$ of the reaction of 1a catalyzed by (thiolfan*)Zr( $\left.\mathrm{NEt}_{2}\right)_{2}$ (Table 1, entry 1): $\delta 1.02$ (d, 3H CH$\left.H_{3}\right), 1.82\left(\mathrm{dd}, 1 \mathrm{H}, \mathrm{NHCHCH}_{2}\right), 2.38$ (dd, $\left.1 \mathrm{H}, \mathrm{NCHCH}_{2}\right), 3.14\left(\mathrm{~m}, 1 \mathrm{H}, \mathrm{NHCHCH}_{3}\right), 3.35$ (dd, 1H, $\left.\mathrm{NHCH}_{2}\right), 3.53\left(\mathrm{dd}, 2 \mathrm{H}, \mathrm{NHCH}_{2}\right), 7.12$ (m, $10 \mathrm{H} \mathrm{Ar} H$ ).

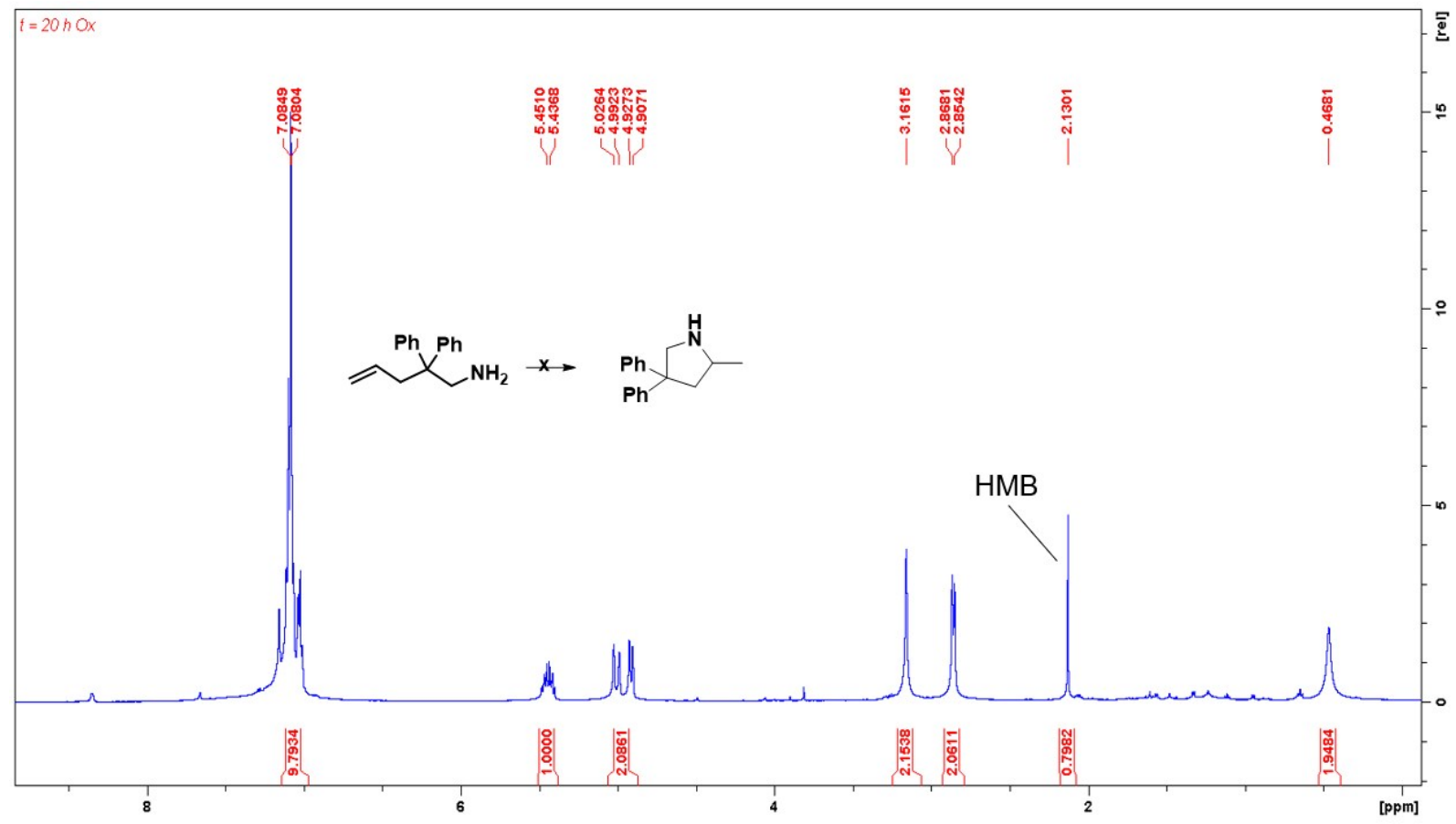

Figure S10: ${ }^{1} \mathrm{H}$ NMR spectrum $\left(\mathrm{C}_{6} \mathrm{D}_{6}, 300 \mathrm{MHz}, 298 \mathrm{~K}\right)$ of 1 a catalyzed by the oxidized species (Table 1, entry 2): $\delta 0.46\left(\mathrm{~s}, 2 \mathrm{H} \mathrm{NH} \mathrm{H}_{2}\right), 2.88\left(\mathrm{~d}, 2 \mathrm{H}, \mathrm{CHCH}_{2}\right), 3.16\left(\mathrm{~s}, 2 \mathrm{H}, \mathrm{NH}_{2} \mathrm{CH}_{2}\right), 5.02(\mathrm{~m}, 2 \mathrm{H}$, $\left.\mathrm{CH}_{2}=\mathrm{CH}\right), 5.45\left(\mathrm{~m}, 1 \mathrm{H}, \mathrm{CH}_{2}=\mathrm{CH}\right) 7.08(\mathrm{~m}, 10 \mathrm{H} \mathrm{ArH})$. 


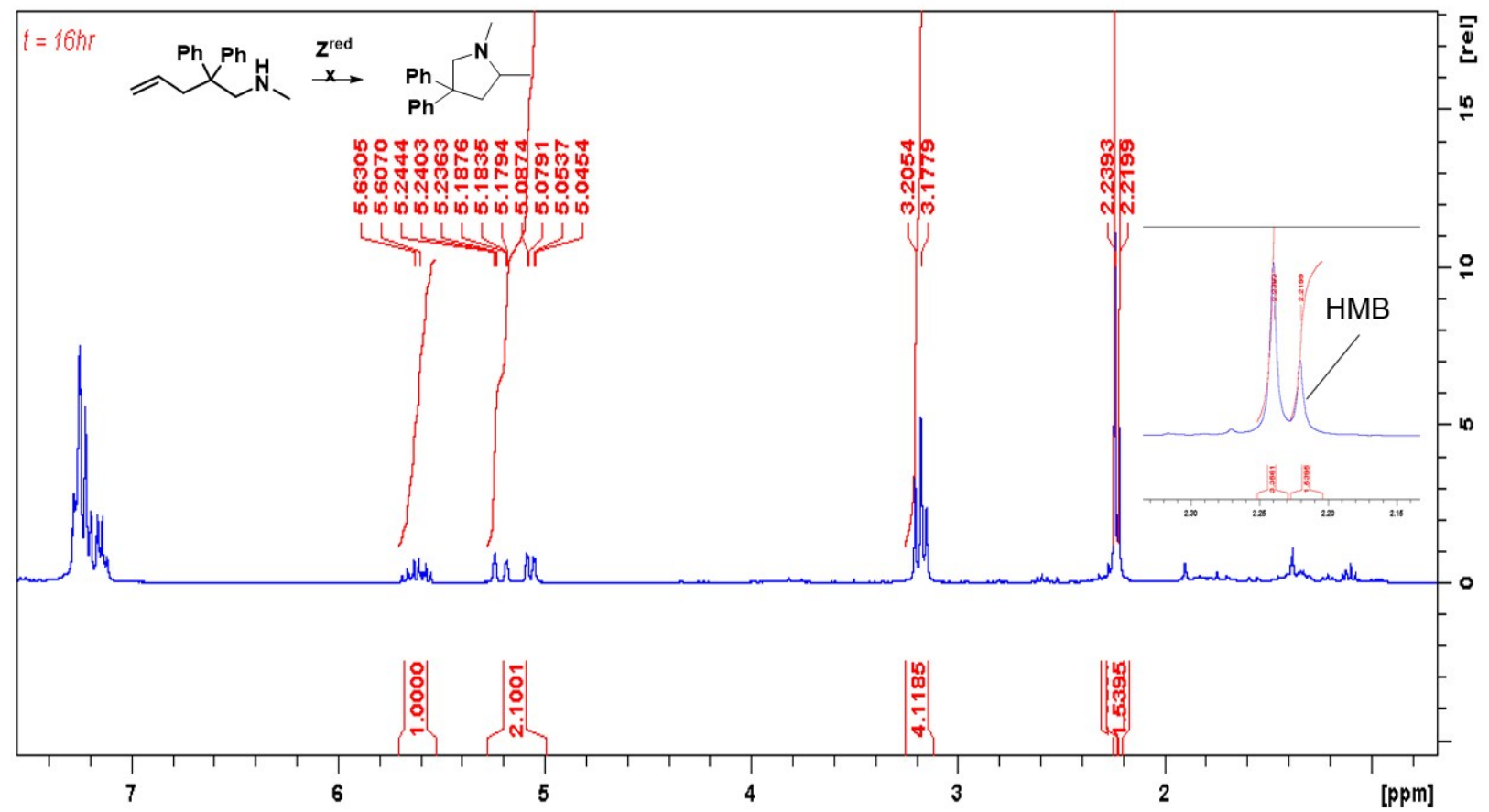

Figure S11: ${ }^{1} \mathrm{H}$ NMR spectrum $\left(\mathrm{C}_{6} \mathrm{D}_{6}, 500 \mathrm{MHz}, 298 \mathrm{~K}\right)$ of $\mathbf{2 a}$ catalyzed by (thiolfan*) $\mathrm{Zr}\left(\mathrm{NEt}_{2}\right)_{2}$ (Table 1, entry 3): $\delta 2.23\left(\mathrm{~s}, 3 \mathrm{H}, \mathrm{NHCH}_{3}\right), 3.17\left(\mathrm{~s}, 2 \mathrm{H}, \mathrm{NHCH}_{2}\right), 3.20\left(\mathrm{~d}, 2 \mathrm{H}, \mathrm{CHCH}_{2}\right), 5.18(\mathrm{~m}$, $\left.2 \mathrm{H}, \mathrm{CH}_{2}=\mathrm{CH}\right), 5.63\left(\mathrm{~m}, 1 \mathrm{H}, \mathrm{CH}_{2}=\mathrm{CH}\right)$.

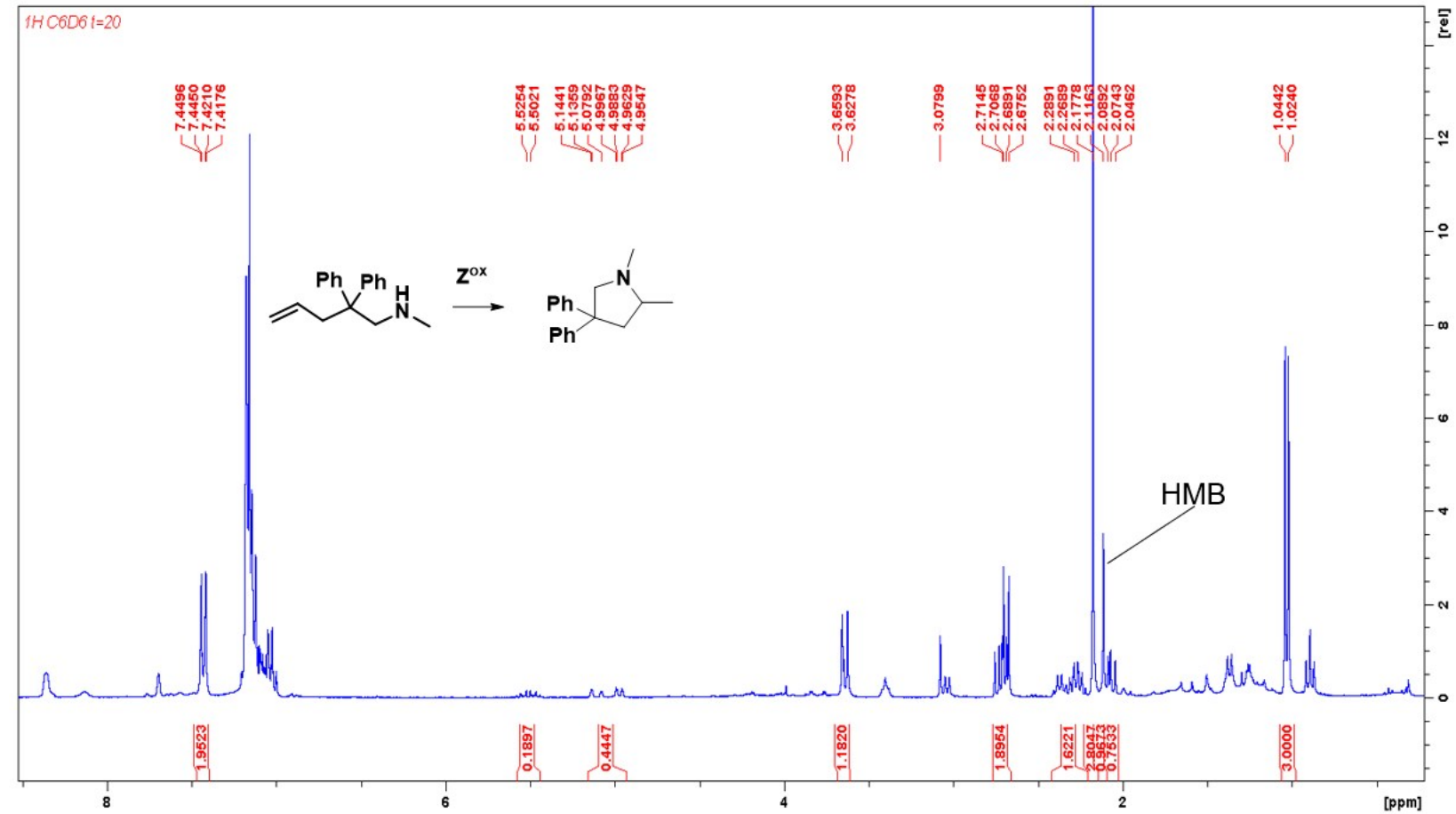

Figure S12: ${ }^{1} \mathrm{H}$ NMR spectrum $\left(\mathrm{C}_{6} \mathrm{D}_{6}, 500 \mathrm{MHz}, 298 \mathrm{~K}\right)$ of $\mathbf{2 a}$ catalyzed by the oxidized species (Table 1, entry 4): $\delta 1.04\left(\mathrm{~d}, 3 \mathrm{H}, \mathrm{NCH}_{3}\right), 2.04\left(\mathrm{t}, 1 \mathrm{H}, \mathrm{MeCHCH}_{2}\right), 2.08\left(\mathrm{~s}, 3 \mathrm{H}, \mathrm{NCHCH}_{3}\right), 2.28$ $\left(\mathrm{m}, 1 \mathrm{H}, \mathrm{MeCHCH}\right.$ ), $2.71\left(\mathrm{~m}, 2 \mathrm{H}, \mathrm{NMeCH}_{2}\right), 3.65$ (d, $\left.1 \mathrm{H}, \mathrm{MeCHCH}_{2}\right)$. 


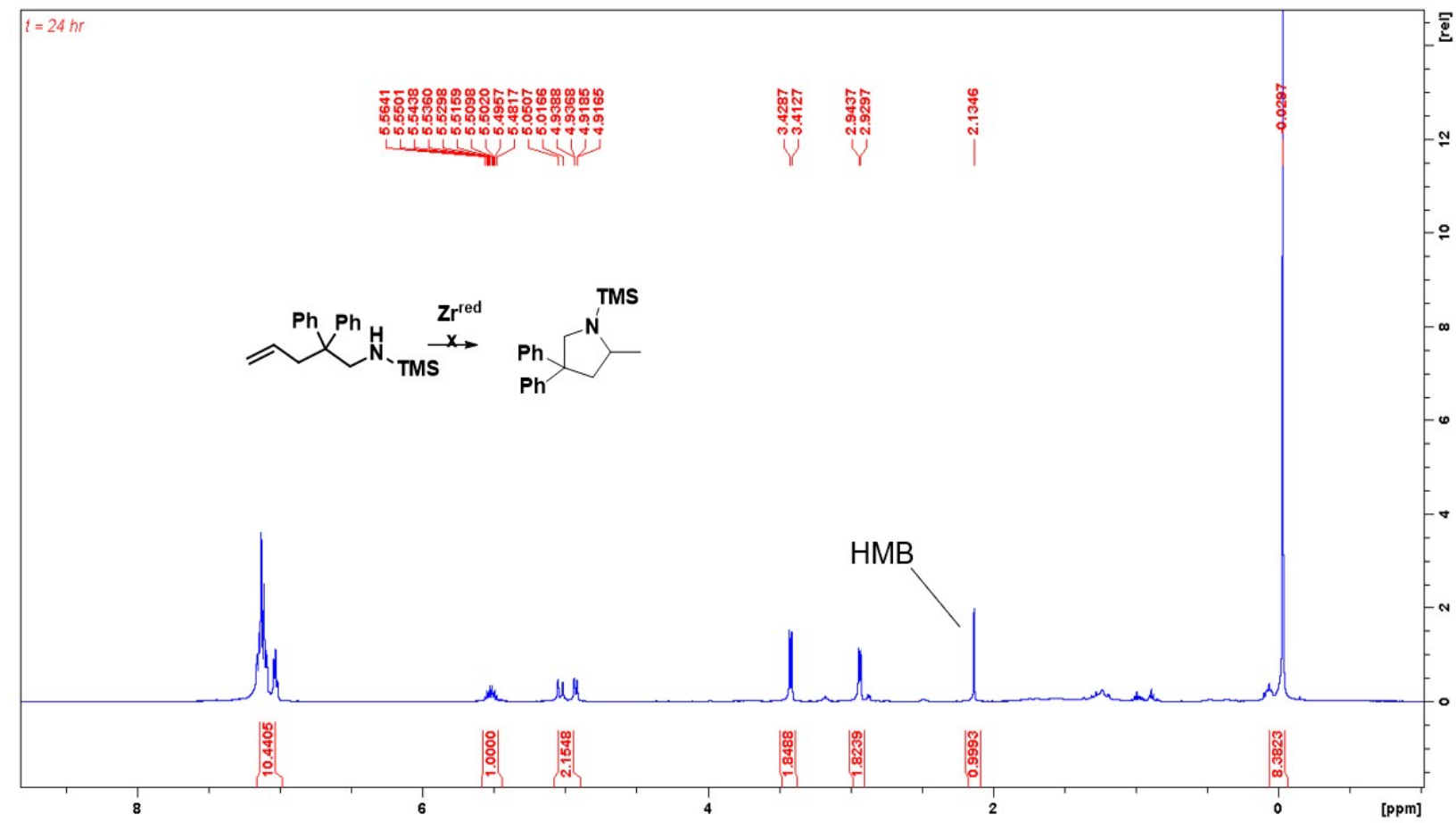

Figure S13: ${ }^{1} \mathrm{H}$ NMR spectrum $\left(\mathrm{C}_{6} \mathrm{D}_{6}, 500 \mathrm{MHz}, 298 \mathrm{~K}\right)$ of $\mathbf{3 a}$ catalyzed by (thiolfan*)Zr( $\left.\mathrm{NEt}_{2}\right)_{2}$ (Table 1, entry 5): $\delta 0.02\left(\mathrm{~s}, 9 \mathrm{H}, \mathrm{SiC}\left(\mathrm{CH}_{3}\right)_{3}\right), 2094\left(\mathrm{~d}, 2 \mathrm{H}, \mathrm{NHCH}_{2}\right), 3.42\left(\mathrm{~d}, 2 \mathrm{H}, \mathrm{CHCH}_{2}\right), 5.05$ $\left(\mathrm{m}, 2 \mathrm{H}, \mathrm{CH}_{2}=\mathrm{CH}\right), 5.56\left(\mathrm{~m}, 1 \mathrm{H}, \mathrm{CH}_{2}=\mathrm{CH}\right), 7.14(\mathrm{~m}, 10 \mathrm{H}, \mathrm{ArH})$.

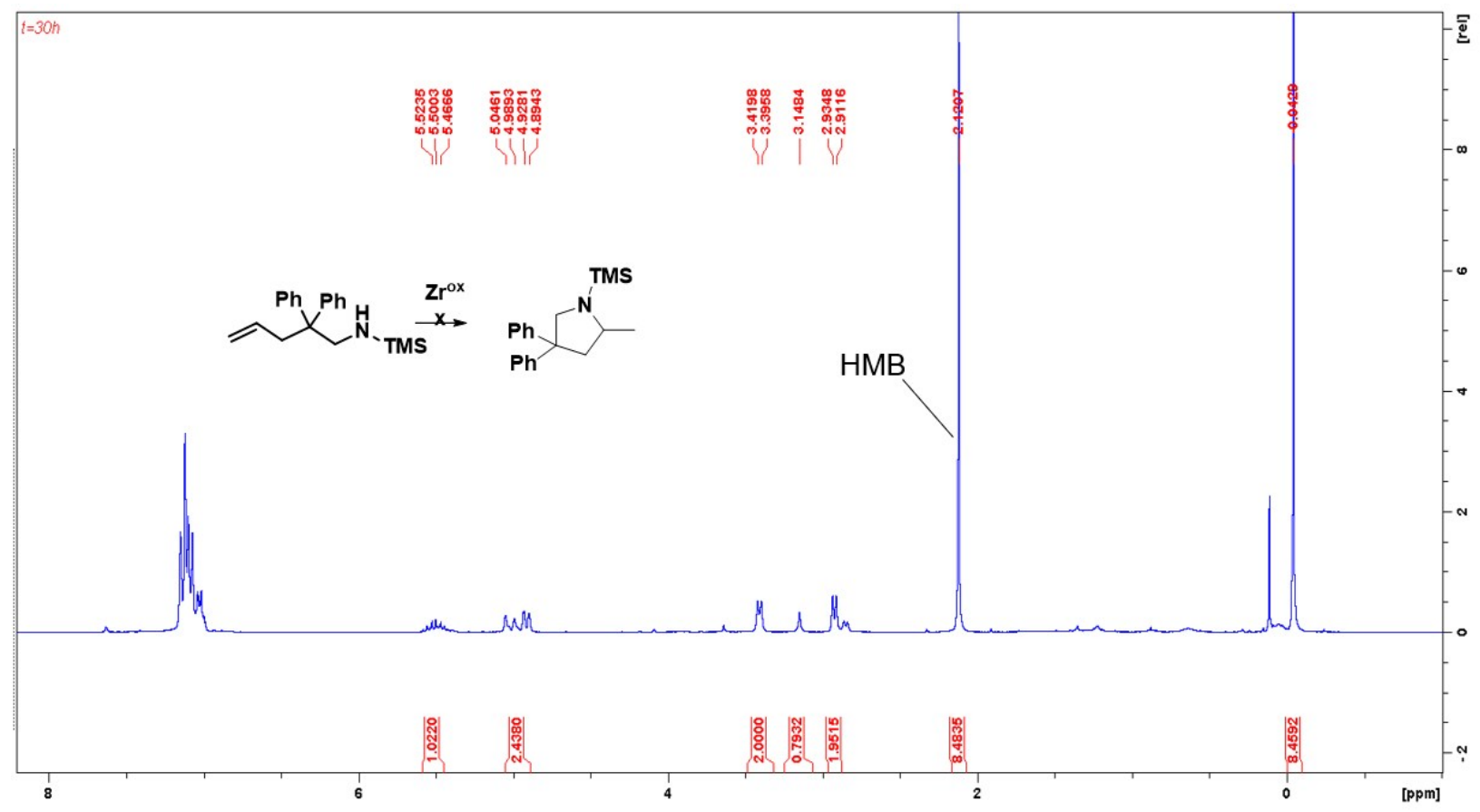

Figure S14: ${ }^{1} \mathrm{H}$ NMR spectrum $\left(\mathrm{C}_{6} \mathrm{D}_{6}, 500 \mathrm{MHz}, 298 \mathrm{~K}\right)$ of $\mathbf{3 a}$ catalyzed by the oxidized species (Table 1, entry 6): $\delta 0.02$ (s, 9H, $\left.\mathrm{SiC}\left(\mathrm{CH}_{3}\right)_{3}\right), 2094$ (d, 2H, $\left.\mathrm{NHCH}_{2}\right), 3.42\left(\mathrm{~d}, 2 \mathrm{H}, \mathrm{CHCH}_{2}\right), 5.05$ $\left(\mathrm{m}, 2 \mathrm{H}, \mathrm{CH}_{2}=\mathrm{CH}\right), 5.56\left(\mathrm{~m}, 1 \mathrm{H}, \mathrm{CH}_{2}=\mathrm{CH}\right)$. 


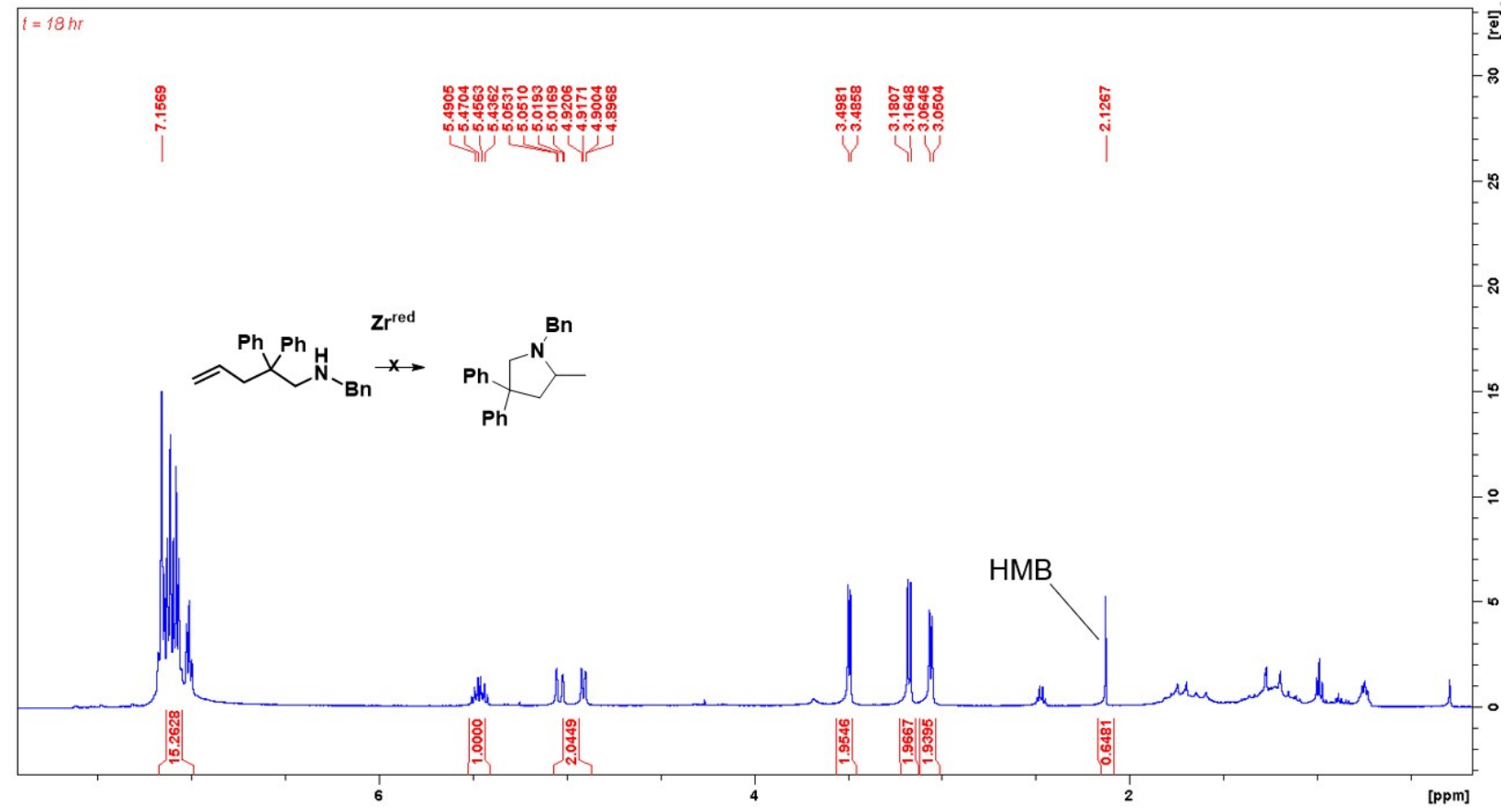

Figure S15: ${ }^{1} \mathrm{H}$ NMR spectrum $\left(\mathrm{C}_{6} \mathrm{D}_{6}, 500 \mathrm{MHz}, 298 \mathrm{~K}\right)$ of 4 a catalyzed by (thiolfan*) $\mathrm{Zr}\left(\mathrm{NEt}_{2}\right)_{2}$ (Table 1, entry 7): $\delta 3.06$ (d, 2H, $\left.\mathrm{CHCH}_{3}\right), 3.18$ (d, 2H, $\left.\mathrm{NHCH}_{2}\right), 3.48\left(\mathrm{~d}, 2 \mathrm{H}, \mathrm{CH}_{2} \mathrm{Ph}\right), 5.05$ (dd, $\left.2 \mathrm{H}, \mathrm{CH}_{2}=\mathrm{CH}\right), 5.49\left(\mathrm{~m}, 1 \mathrm{H}, \mathrm{CH}_{2}=\mathrm{CH}\right), 7.15(\mathrm{~m}, 15 \mathrm{H} \mathrm{ArH})$.

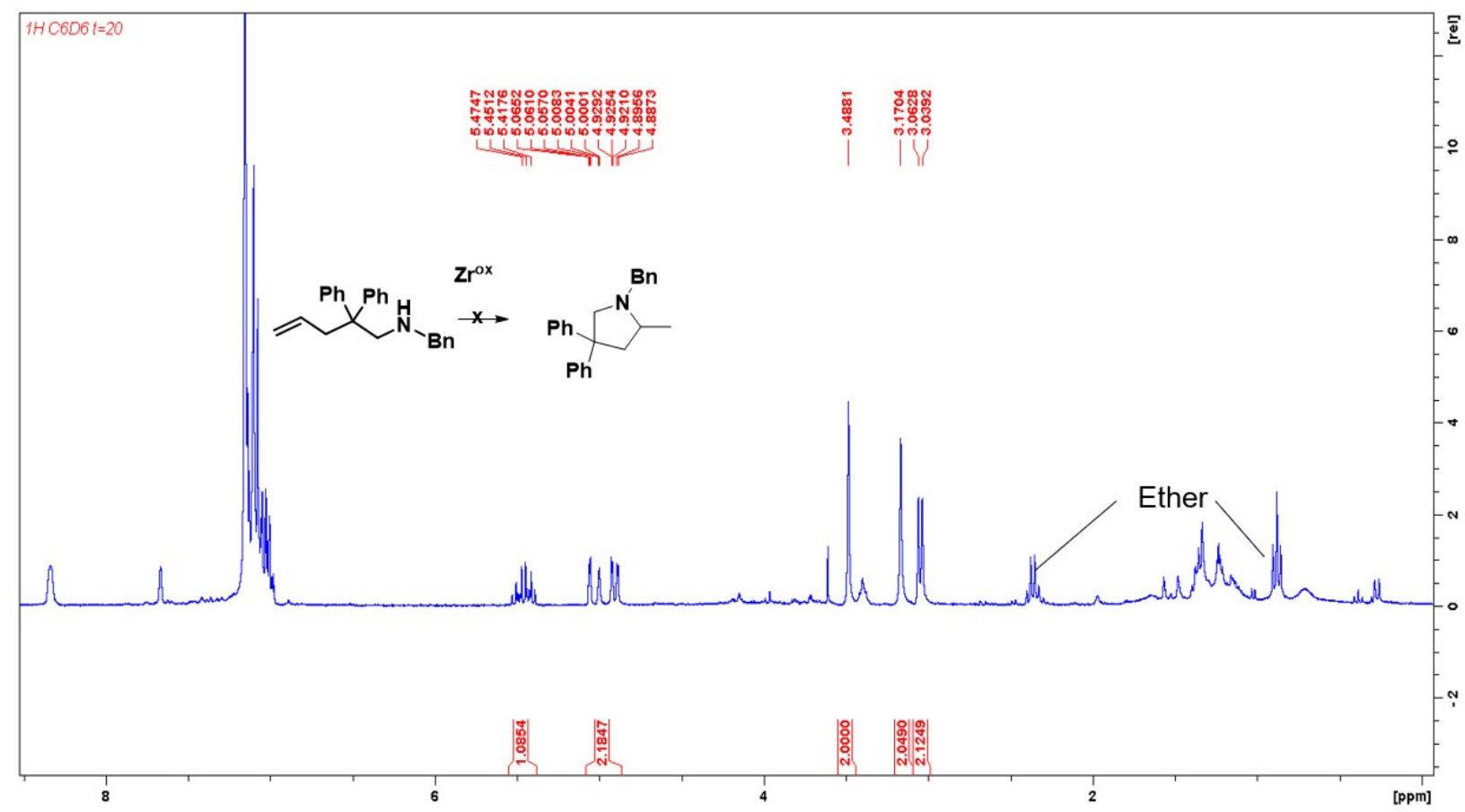

Figure S16: ${ }^{1} \mathrm{H}$ NMR spectrum $\left(\mathrm{C}_{6} \mathrm{D}_{6}, 500 \mathrm{MHz}, 298 \mathrm{~K}\right)$ of 4 a catalyzed by the oxidized species (Table 1, entry 8): $\delta 3.08\left(\mathrm{~d}, 2 \mathrm{H}, \mathrm{CHCH}_{3}\right), 3.17\left(\mathrm{~d}, 2 \mathrm{H}, \mathrm{NHCH}_{2}\right), 3.48\left(\mathrm{~d}, 2 \mathrm{H}, \mathrm{CH}_{2} \mathrm{Ph}\right), 5.05(\mathrm{dd}$, $\left.2 \mathrm{H}, \mathrm{CH}_{2}=\mathrm{CH}\right), 5.49\left(\mathrm{~m}, 1 \mathrm{H}, \mathrm{CH}_{2}=\mathrm{CH}\right), 7.15(\mathrm{~m}, 15 \mathrm{H} \mathrm{ArH})$. 


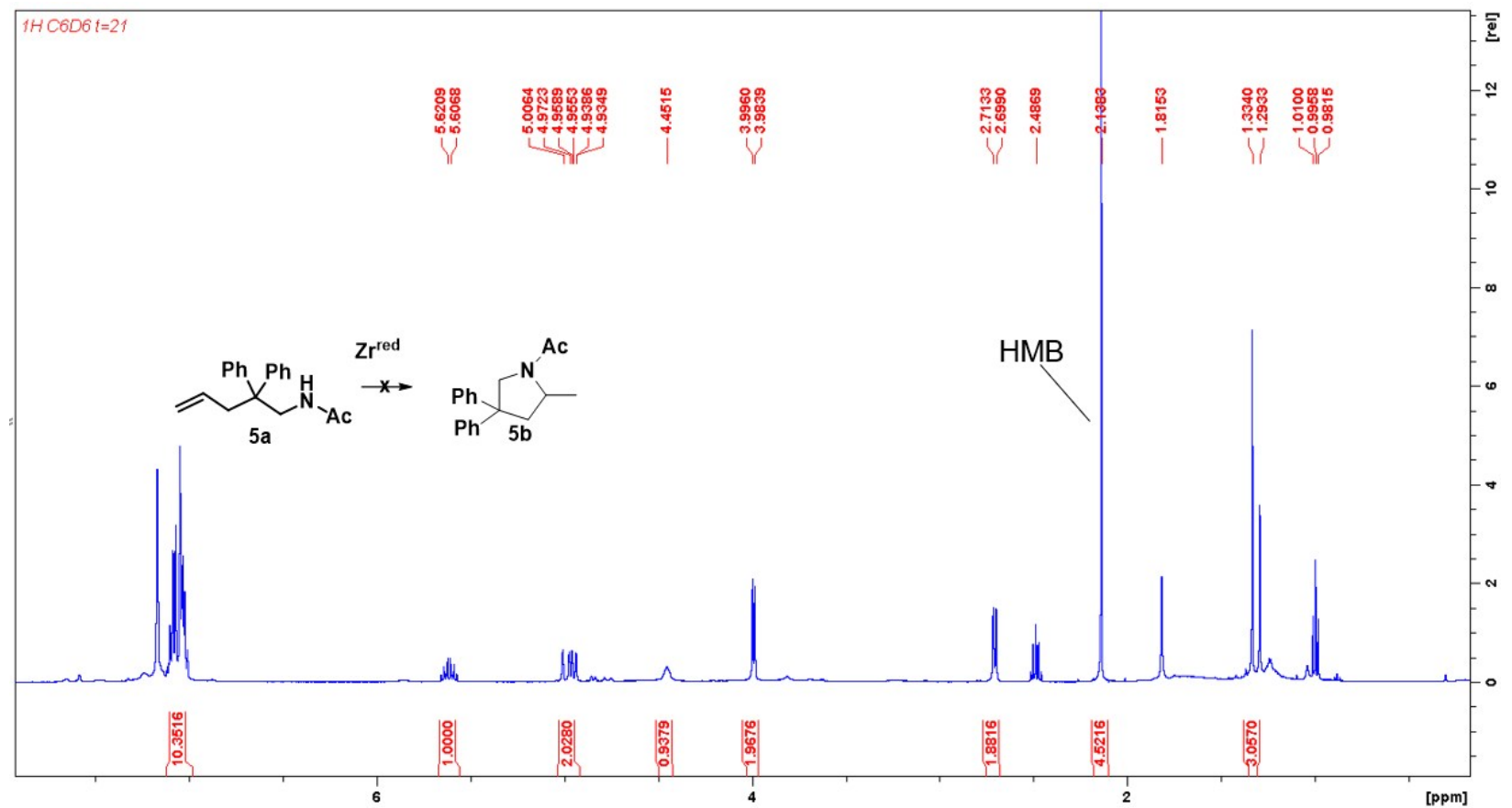

Figure S17: ${ }^{1} \mathrm{H}$ NMR spectrum $\left(\mathrm{C}_{6} \mathrm{D}_{6}, 500 \mathrm{MHz}, 298 \mathrm{~K}\right)$ of $\mathbf{5 a}$ catalyzed by (thiolfan*) $\mathrm{Zr}\left(\mathrm{NEt}_{2}\right)_{2}$ (Table 1, entry 9): $\delta 1.33$ (s, 3H, $\mathrm{COCH}_{3}$ ), 2.71 (d, 2H, $\mathrm{NHCH}_{2}$ ), 3.99 (d, 2H, $\mathrm{CHCH}_{2}$ ), 5.00 (dd, $\left.2 \mathrm{H}, \mathrm{CH}_{2}=\mathrm{CH}\right), 5.62\left(\mathrm{~m}, 1 \mathrm{H}, \mathrm{CH}_{2}=\mathrm{CH}\right), 7.15(\mathrm{~m}, 10 \mathrm{H} \mathrm{ArH})$. The peaks at 1.01 and $2.48 \mathrm{ppm}$ are attributed to residual triethylamine.

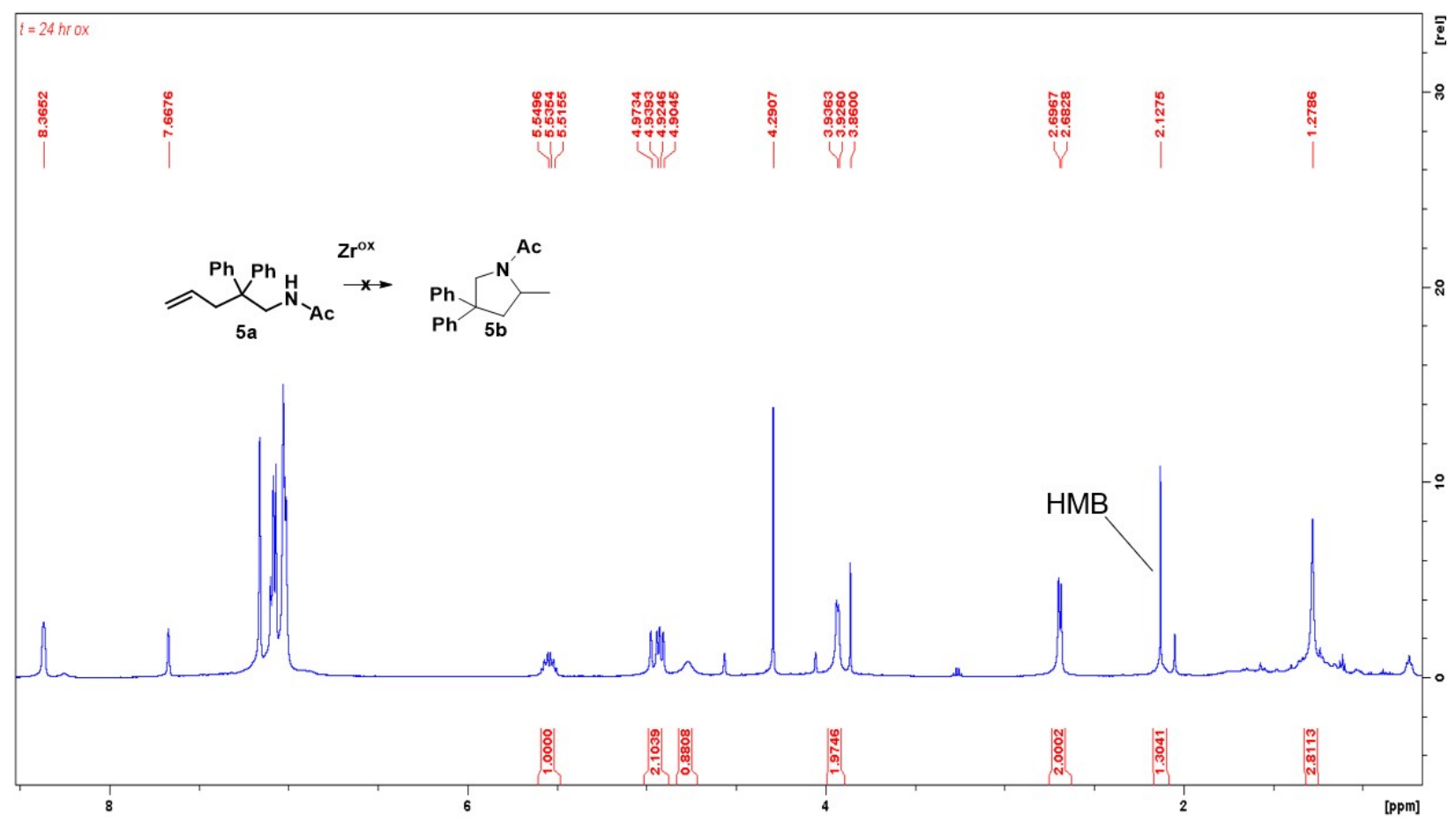

Figure S18: ${ }^{1} \mathrm{H}$ NMR spectrum $\left(\mathrm{C}_{6} \mathrm{D}_{6}, 500 \mathrm{MHz}, 298 \mathrm{~K}\right)$ of $\mathbf{5 a}$ catalyzed by the oxidized species (Table 1, entry 10): $\delta 1.27\left(\mathrm{~s}, 3 \mathrm{H}, \mathrm{COCH}_{3}\right), 2.69\left(\mathrm{~d}, 2 \mathrm{H}, \mathrm{NHCH}_{2}\right), 3.93\left(\mathrm{~d}, 2 \mathrm{H}, \mathrm{CHCH}_{2}\right), 4.97$ $\left(\mathrm{dd}, 2 \mathrm{H}, \mathrm{CH}_{2}=\mathrm{CH}\right), 5.54\left(\mathrm{~m}, 1 \mathrm{H}, \mathrm{CH}_{2}=\mathrm{CH}\right)$. The peak at $4.29 \mathrm{ppm}$ is attributed to residual dichloromethane (DCM). 


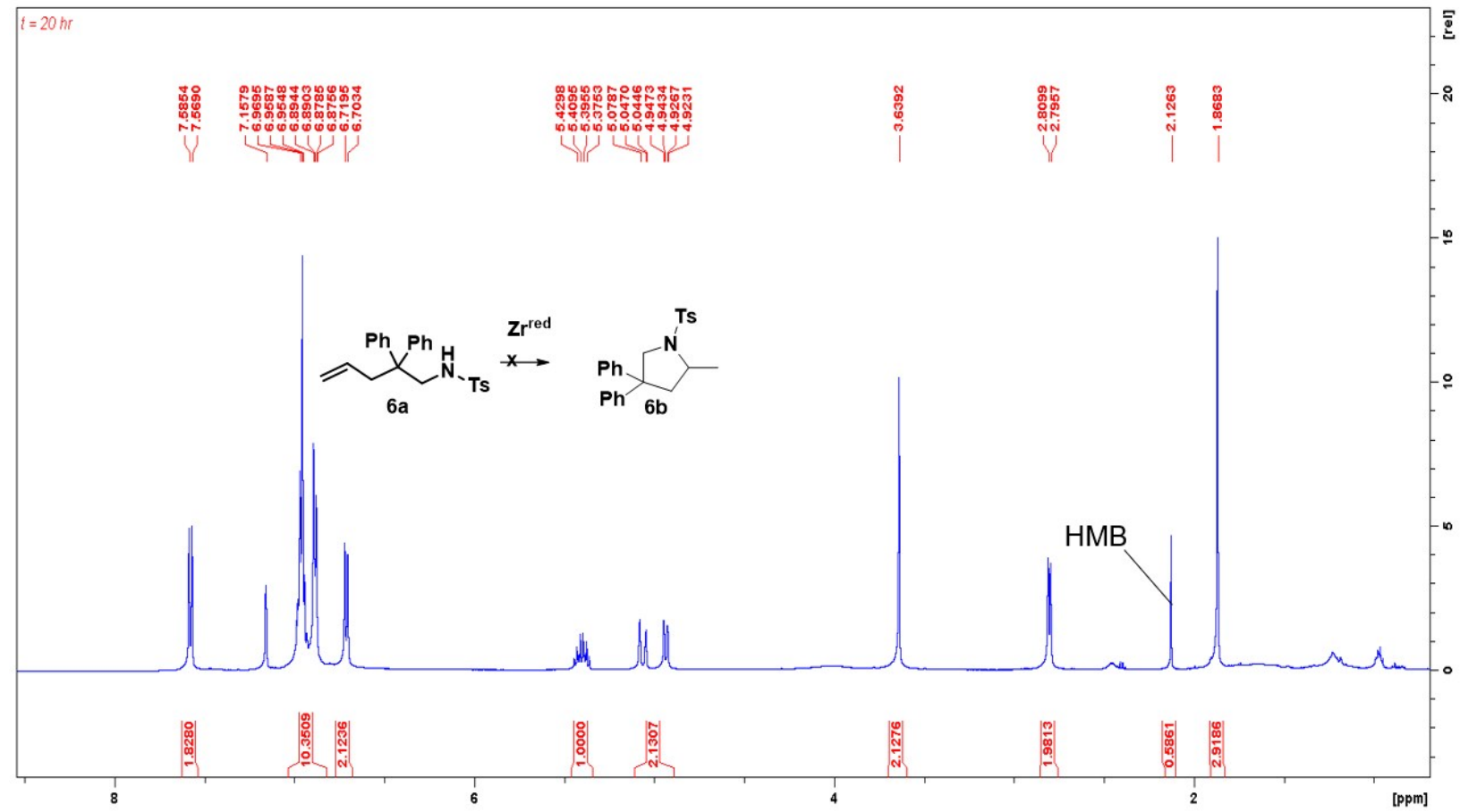

Figure S19: ${ }^{1} \mathrm{H}$ NMR spectrum $\left(\mathrm{C}_{6} \mathrm{D}_{6}, 500 \mathrm{MHz}, 298 \mathrm{~K}\right)$ of 6 a catalyzed by (thiolfan*) $\mathrm{Zr}\left(\mathrm{NEt}_{2}\right)_{2}$ (Table 1, entry 11): $\delta 1.86\left(\mathrm{~s}, 3 \mathrm{H}, \mathrm{PhCH}_{3}\right), 2.80$ (d, 2H, $\left.\mathrm{CHCH}_{2}\right), 3.63\left(\mathrm{~s}, 2 \mathrm{H}, \mathrm{NHCH}_{2}\right), 5.05$ (dd, $\left.2 \mathrm{H}, \mathrm{CH}_{2}=\mathrm{CH}\right), 5.42\left(\mathrm{~m}, 1 \mathrm{H}, \mathrm{CH}_{2}=\mathrm{CH}\right), 6.71(\mathrm{~d}, 2 \mathrm{H} \mathrm{ArH}), 7.15(\mathrm{~m}, 15 \mathrm{H} \mathrm{ArH}), 7.58(\mathrm{~d}, 2 \mathrm{H} \mathrm{ArH})$.

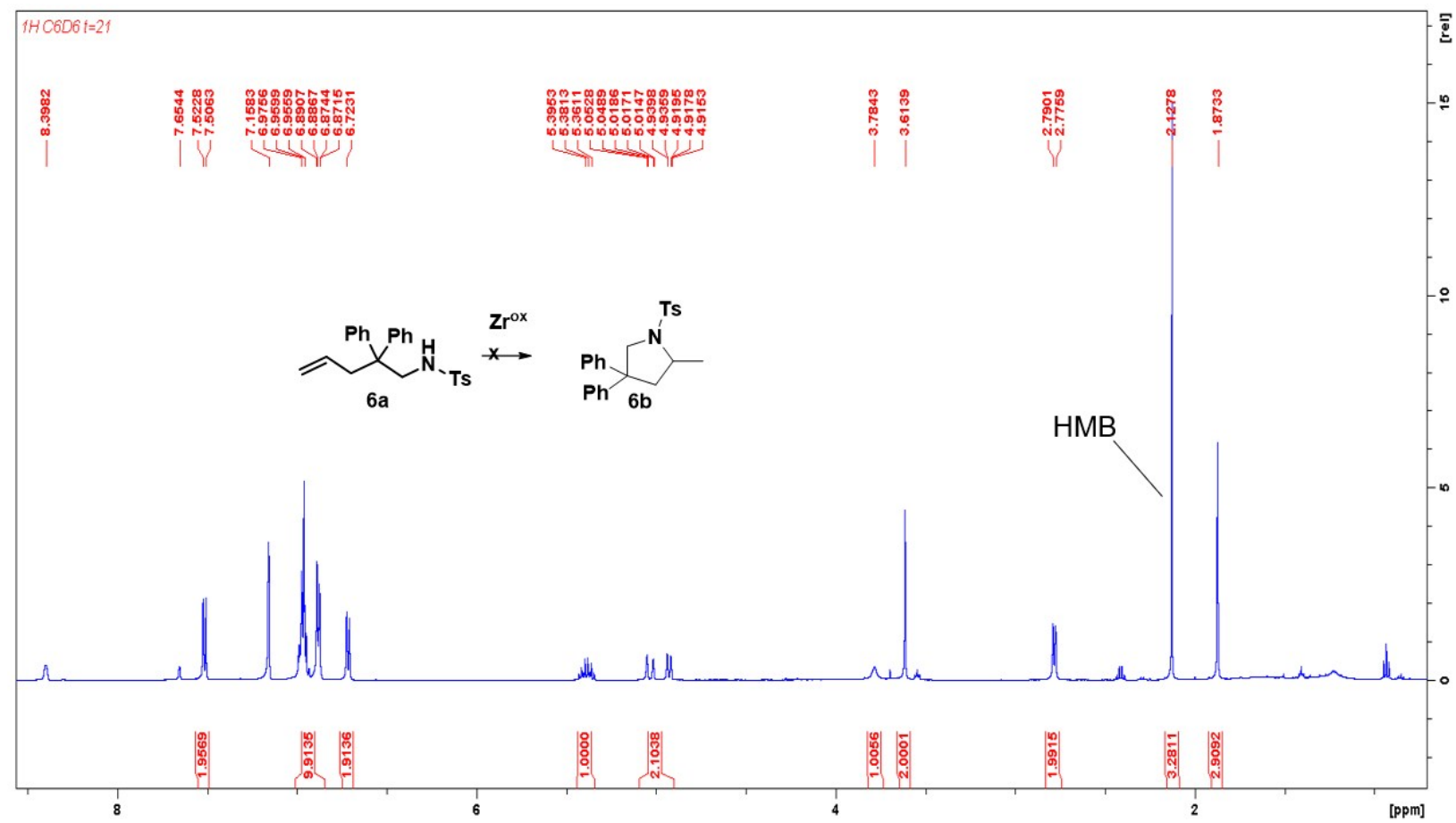

Figure S20: ${ }^{1} \mathrm{H}$ NMR spectrum $\left(\mathrm{C}_{6} \mathrm{D}_{6}, 500 \mathrm{MHz}, 298 \mathrm{~K}\right)$ of 6 a catalyzed by the oxidized species (Table 1 entry 12): $\delta 1.86$ (s, 3H, $\left.\mathrm{PhCH}_{3}\right), 2.80$ (d, 2H, $\left.\mathrm{CHCH}_{2}\right), 3.63$ (s, 2H, $\mathrm{NHCH}_{2}$ ), 3.78 (br, $1 \mathrm{H}, \mathrm{NHTs}), 5.05\left(\mathrm{dd}, 2 \mathrm{H}, \mathrm{CH}_{2}=\mathrm{CH}\right), 5.42\left(\mathrm{~m}, 1 \mathrm{H}, \mathrm{CH}_{2}=\mathrm{CH}\right), 6.71(\mathrm{~d}, 2 \mathrm{H} \mathrm{ArH}), 7.15(\mathrm{~m}, 15 \mathrm{H}$ $\operatorname{ArH}), 7.58(\mathrm{~d}, 2 \mathrm{H} \mathrm{ArH})$. 


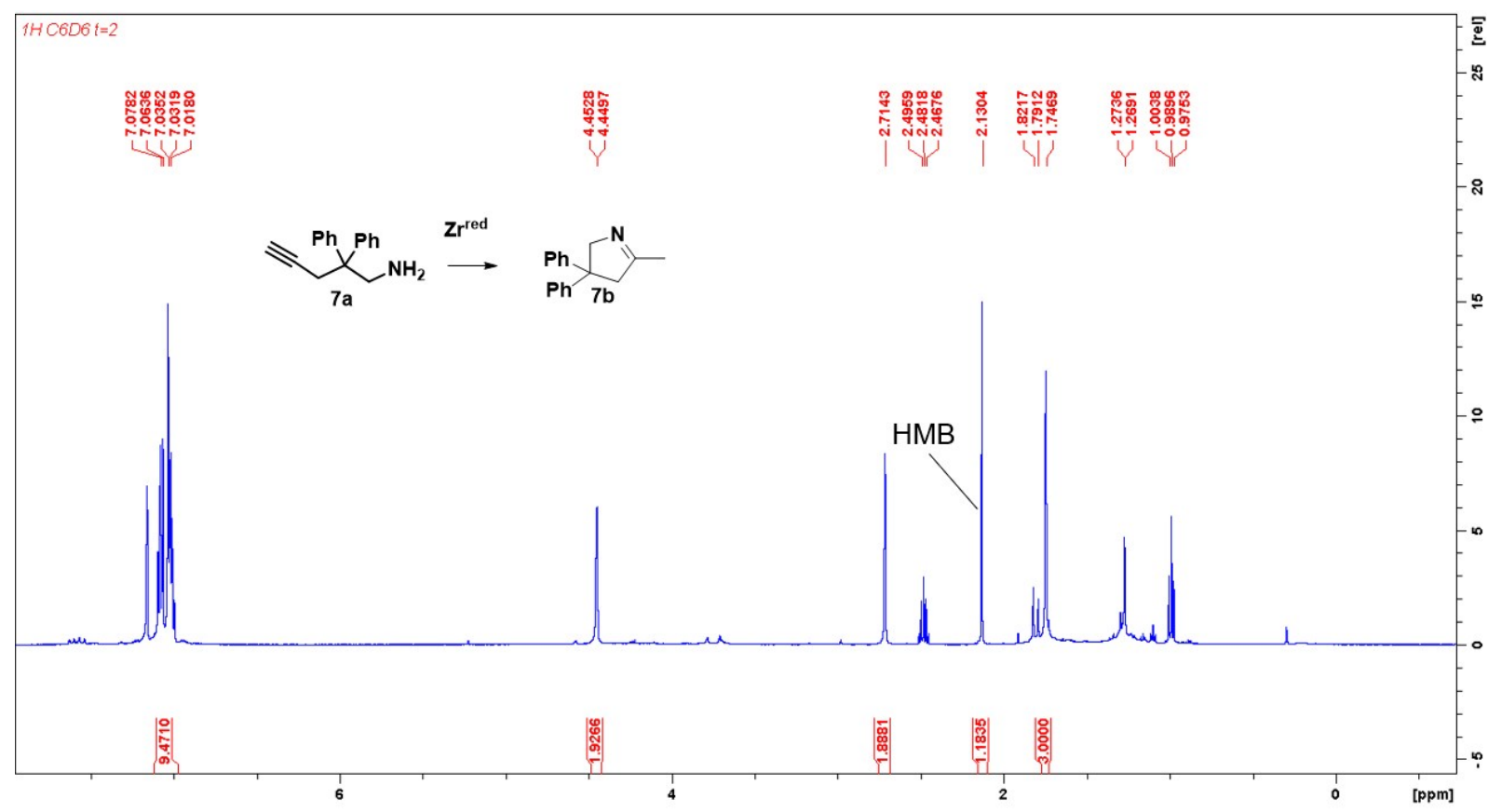

Figure S21: ${ }^{1} \mathrm{H}$ NMR spectrum $\left(\mathrm{C}_{6} \mathrm{D}_{6}, 500 \mathrm{MHz}, 298 \mathrm{~K}\right)$ of $7 \mathbf{a}$ catalyzed by (thiolfan*) $\mathrm{Zr}\left(\mathrm{NEt}_{2}\right)_{2}$ (Table 1, entry 13): $\delta 1.79\left(\mathrm{~s}, 3 \mathrm{H}, \mathrm{CCH}_{3}\right), 2.71\left(\mathrm{~s}, 2 \mathrm{H}, \mathrm{CCH}_{2}\right), 4.45\left(\mathrm{~s}, 2 \mathrm{H}, \mathrm{NCH}_{2}\right), 7.07(\mathrm{~m}, 10 \mathrm{H}$ $\mathrm{ArH})$. The peaks at 1.00 and $2.49 \mathrm{ppm}$ are attributed to triethylamine.

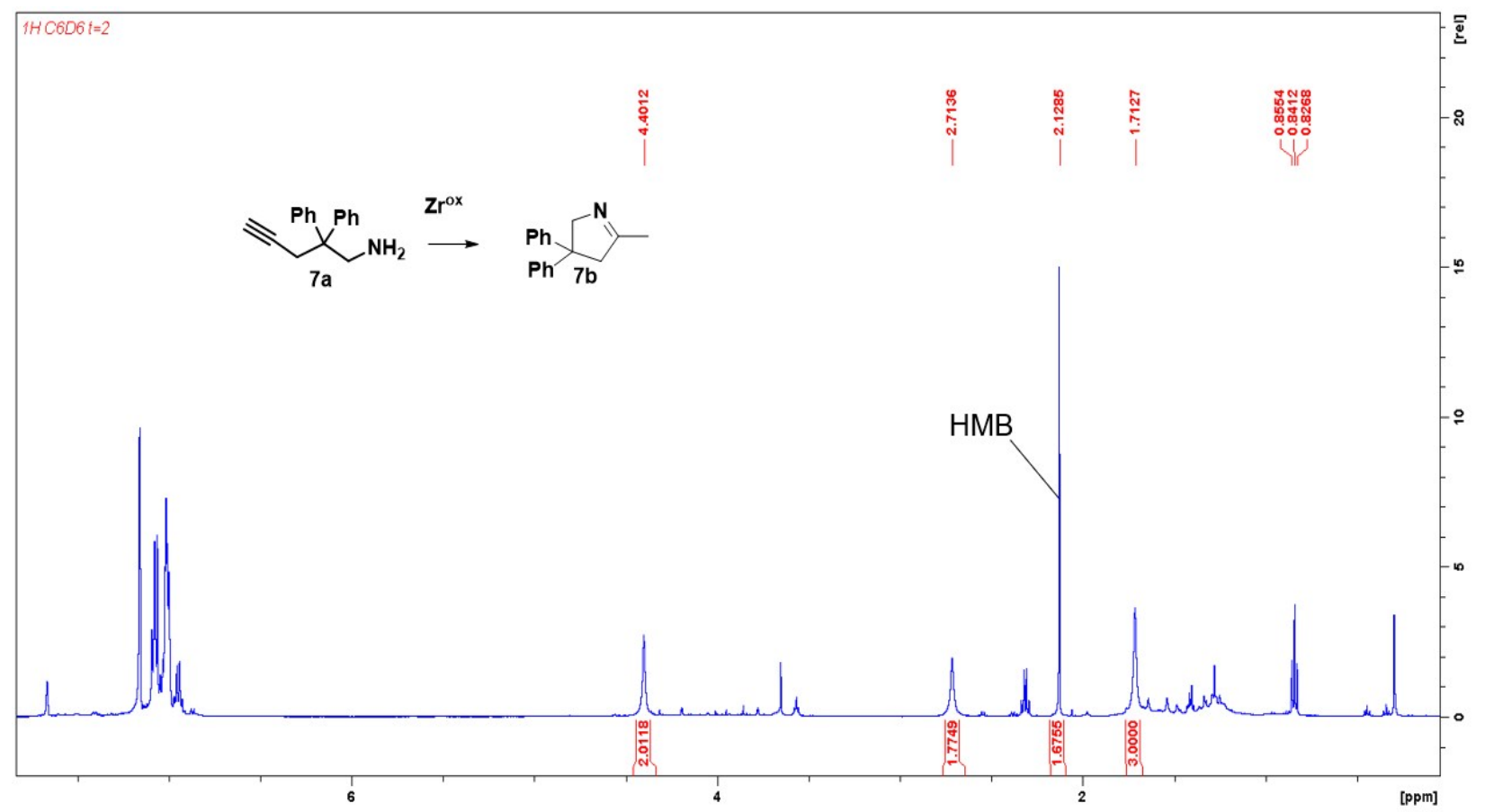

Figure S22: ${ }^{1} \mathrm{H}$ NMR spectrum $\left(\mathrm{C}_{6} \mathrm{D}_{6}, 500 \mathrm{MHz}, 298 \mathrm{~K}\right)$ of $7 \mathbf{a}$ catalyzed by the oxidized species (Table 1, entry 14): $\delta 1.79\left(\mathrm{~s}, 3 \mathrm{H}, \mathrm{CCH}_{3}\right), 2.71\left(\mathrm{~s}, 2 \mathrm{H}, \mathrm{CCH}_{2}\right), 4.45\left(\mathrm{~s}, 2 \mathrm{H}, \mathrm{NCH}_{2}\right)$. 


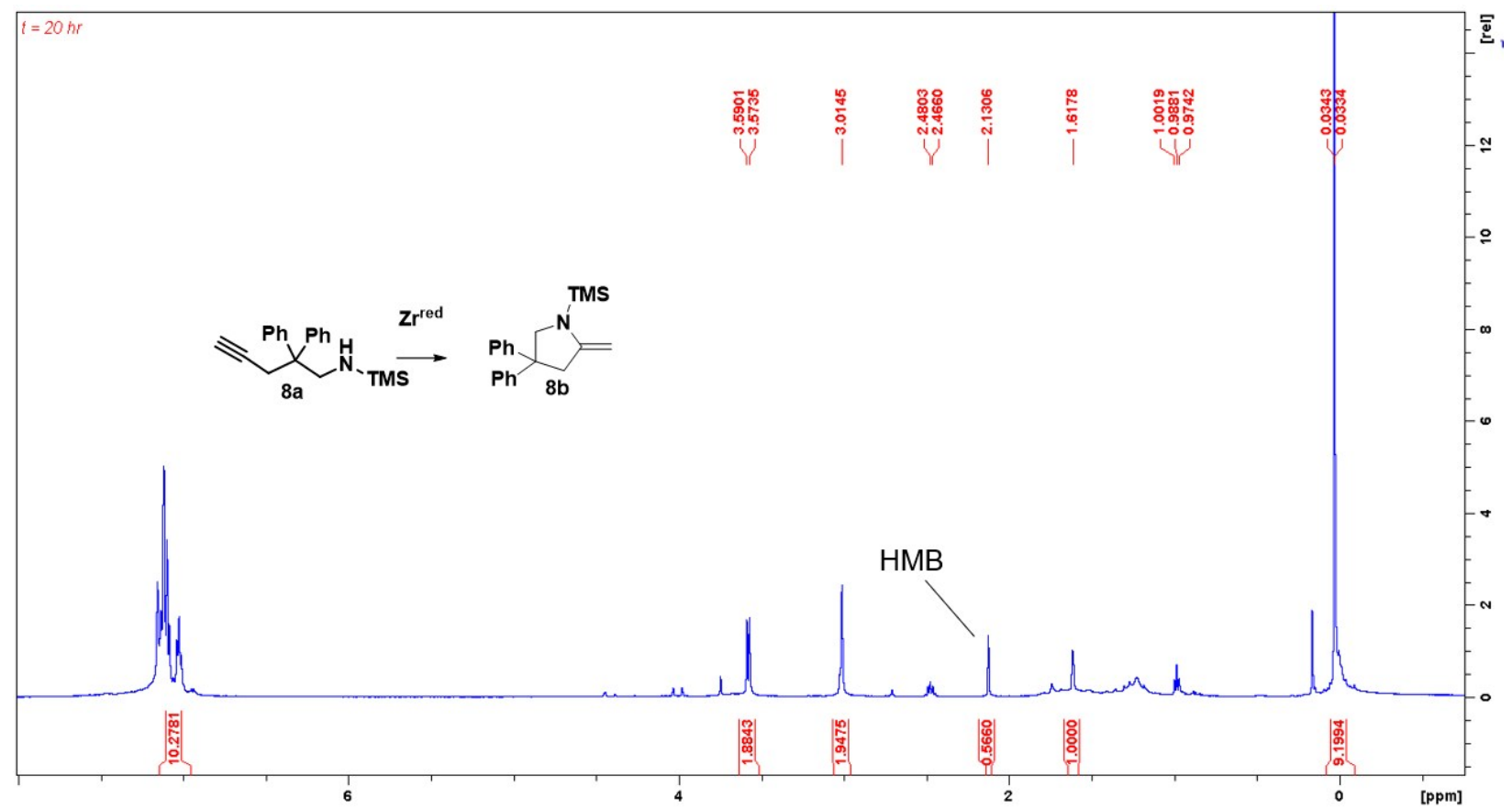

Figure S23: ${ }^{1} \mathrm{H}$ NMR spectrum $\left(\mathrm{C}_{6} \mathrm{D}_{6}, 500 \mathrm{MHz}, 298 \mathrm{~K}\right)$ of 8 a catalyzed by (thiolfan*) $\mathrm{Zr}\left(\mathrm{NEt}_{2}\right)_{2}$ (Table 1, entry 15): $\delta 0.03\left(\mathrm{~s}, 9 \mathrm{H}, \mathrm{SiC}\left(\mathrm{CH}_{3}\right)_{3}\right), 1.61(\mathrm{~s}, 1 \mathrm{H}, \mathrm{C}=\mathrm{CH}), 3.01\left(\mathrm{~s}, 2 \mathrm{H}, \mathrm{NHCH}_{2}\right), 3.59$ (d, $\left.2 \mathrm{H}, \mathrm{CCH}_{2}\right), 7.10(\mathrm{~m}, 10 \mathrm{H}, \mathrm{ArH})$.

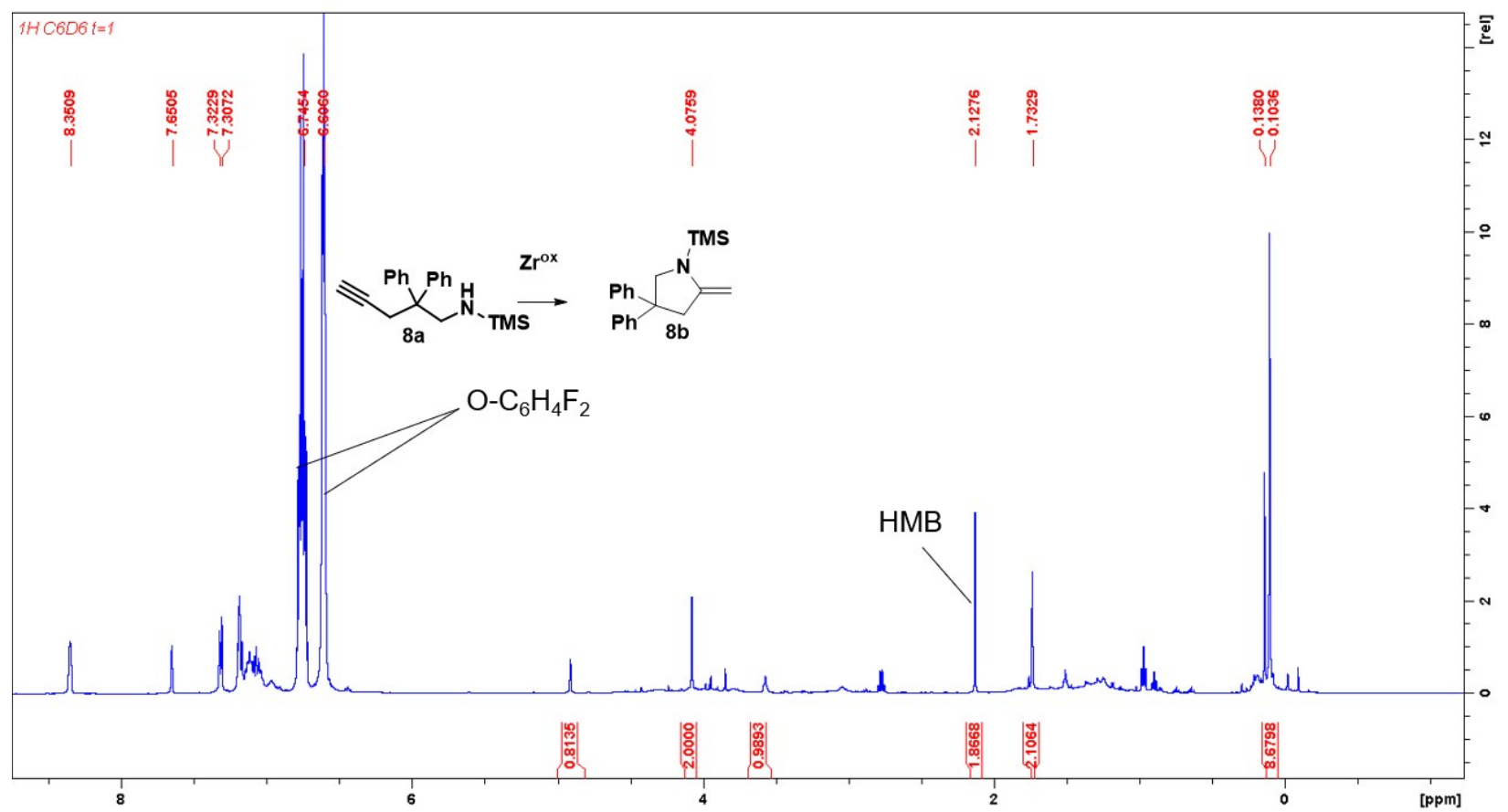

Figure S24: ${ }^{1} \mathrm{H}$ NMR spectrum $\left(\mathrm{C}_{6} \mathrm{D}_{6}, 500 \mathrm{MHz}, 298 \mathrm{~K}\right)$ of $\mathbf{8 a}$ catalyzed by the oxidized species (Table 1, entry 16): $\delta 0.13\left(\mathrm{~s}, 9 \mathrm{H}, \mathrm{SiC}\left(\mathrm{CH}_{3}\right)_{3}\right), 1.73\left(\mathrm{~s}, 2 \mathrm{H}, \mathrm{CCH}_{2}\right), 3.57\left(\mathrm{~s}, 1 \mathrm{H}, \mathrm{C}=\mathrm{CH}_{2}\right), 4.07(\mathrm{~s}$, $\left.2 \mathrm{H}, \mathrm{NCH}_{2}\right) 4.90\left(\mathrm{~s}, 1 \mathrm{H}, \mathrm{C}=\mathrm{CH}_{2}\right)$. 


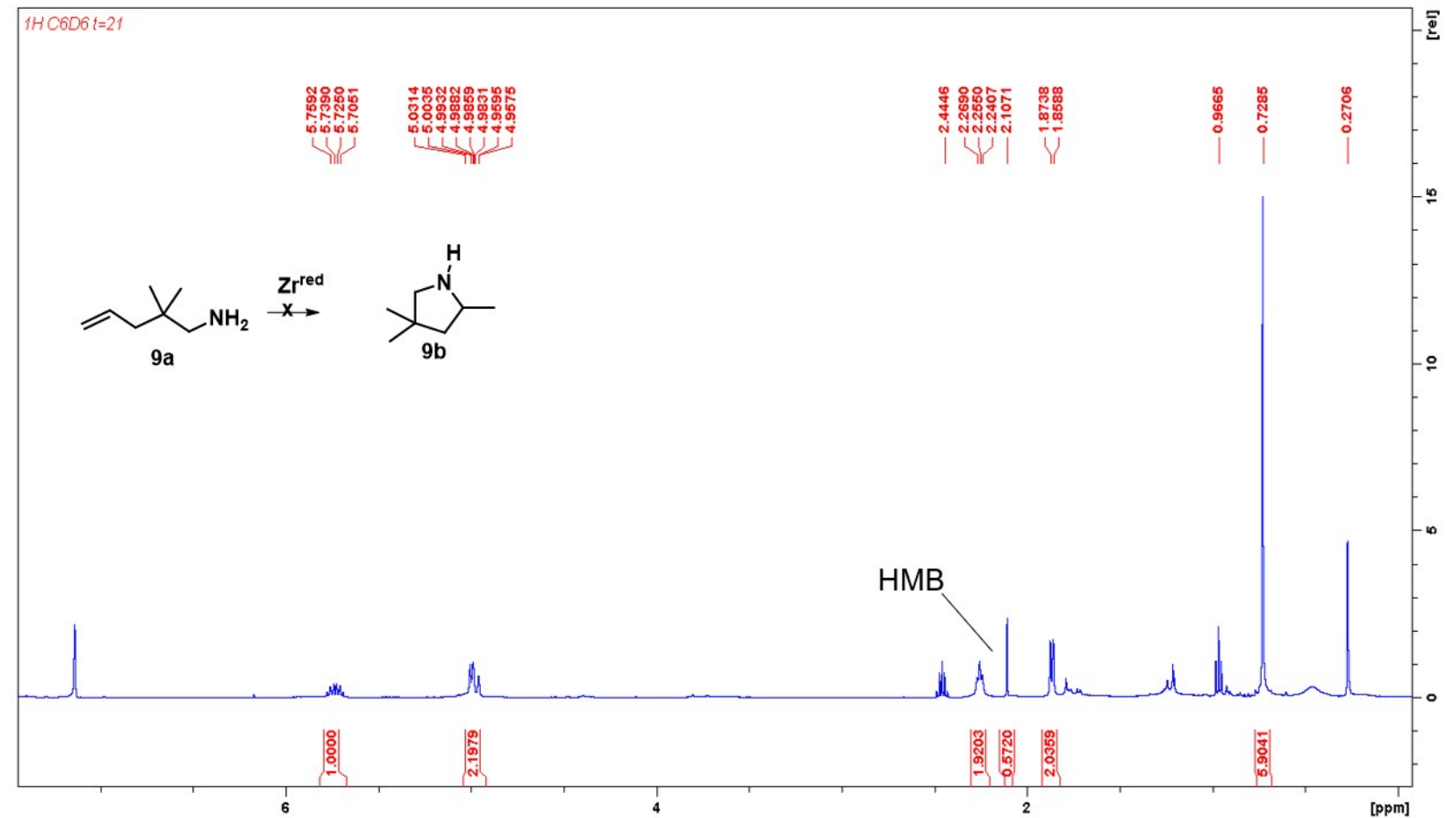

Figure S25: ${ }^{1} \mathrm{H}$ NMR spectrum $\left(\mathrm{C}_{6} \mathrm{D}_{6}, 500 \mathrm{MHz}, 298 \mathrm{~K}\right)$ of 9a catalyzed by (thiolfan*) $\mathrm{Zr}\left(\mathrm{NEt}_{2}\right)_{2}$ (Table 1, entry 17): $\delta 0.72\left(\mathrm{~s}, 6 \mathrm{H}, \mathrm{C}\left(\mathrm{CH}_{3}\right)_{2}\right), 1.87\left(\mathrm{~d}, 2 \mathrm{H}, \mathrm{CHCH}_{2}\right), 2.28\left(\mathrm{t}, 2 \mathrm{H}, \mathrm{NH}_{2} \mathrm{CH}_{2}\right), 4.99$ (m, $\left.2 \mathrm{H}, \mathrm{CH}=\mathrm{CH}_{2}\right) 5.75\left(\mathrm{~m}, 1 \mathrm{H}, \mathrm{CH}=\mathrm{CH}_{2}\right)$.

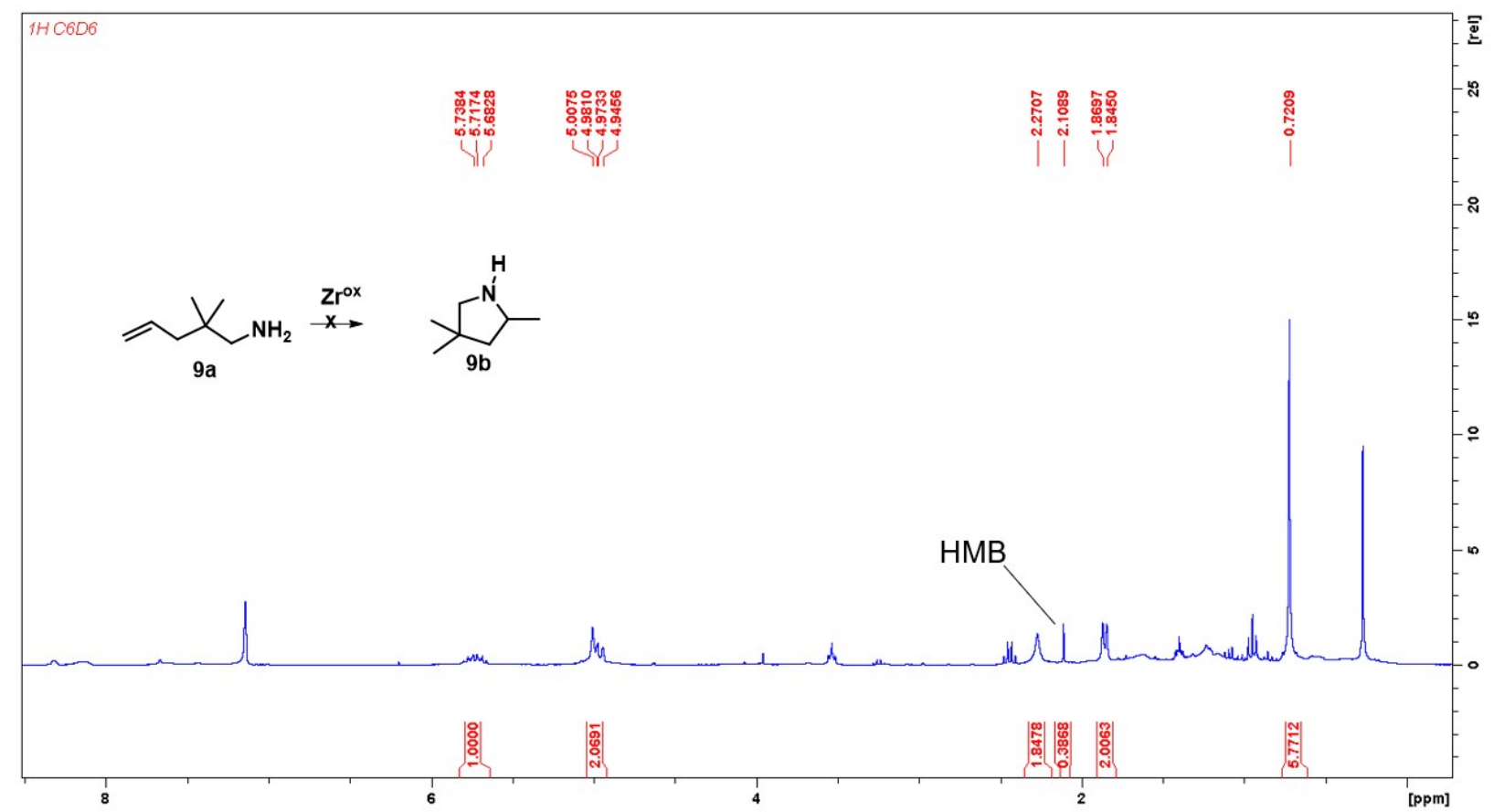

Figure S26: ${ }^{1} \mathrm{H}$ NMR spectrum $\left(\mathrm{C}_{6} \mathrm{D}_{6}, 500 \mathrm{MHz}, 298 \mathrm{~K}\right)$ of 9 a catalyzed by the oxidized species (Table 1, entry 18): $\delta 0.72\left(\mathrm{~s}, 6 \mathrm{H}, \mathrm{C}\left(\mathrm{CH}_{3}\right)_{2}\right), 1.87\left(\mathrm{~d}, 2 \mathrm{H}, \mathrm{CHCH}_{2}\right), 2.28\left(\mathrm{t}, 2 \mathrm{H}, \mathrm{NH}_{2} \mathrm{CH}_{2}\right), 4.99(\mathrm{~m}$, $\left.2 \mathrm{H}, \mathrm{CH}=\mathrm{CH}_{2}\right) 5.75\left(\mathrm{~m}, 1 \mathrm{H}, \mathrm{CH}=\mathrm{CH}_{2}\right)$. 


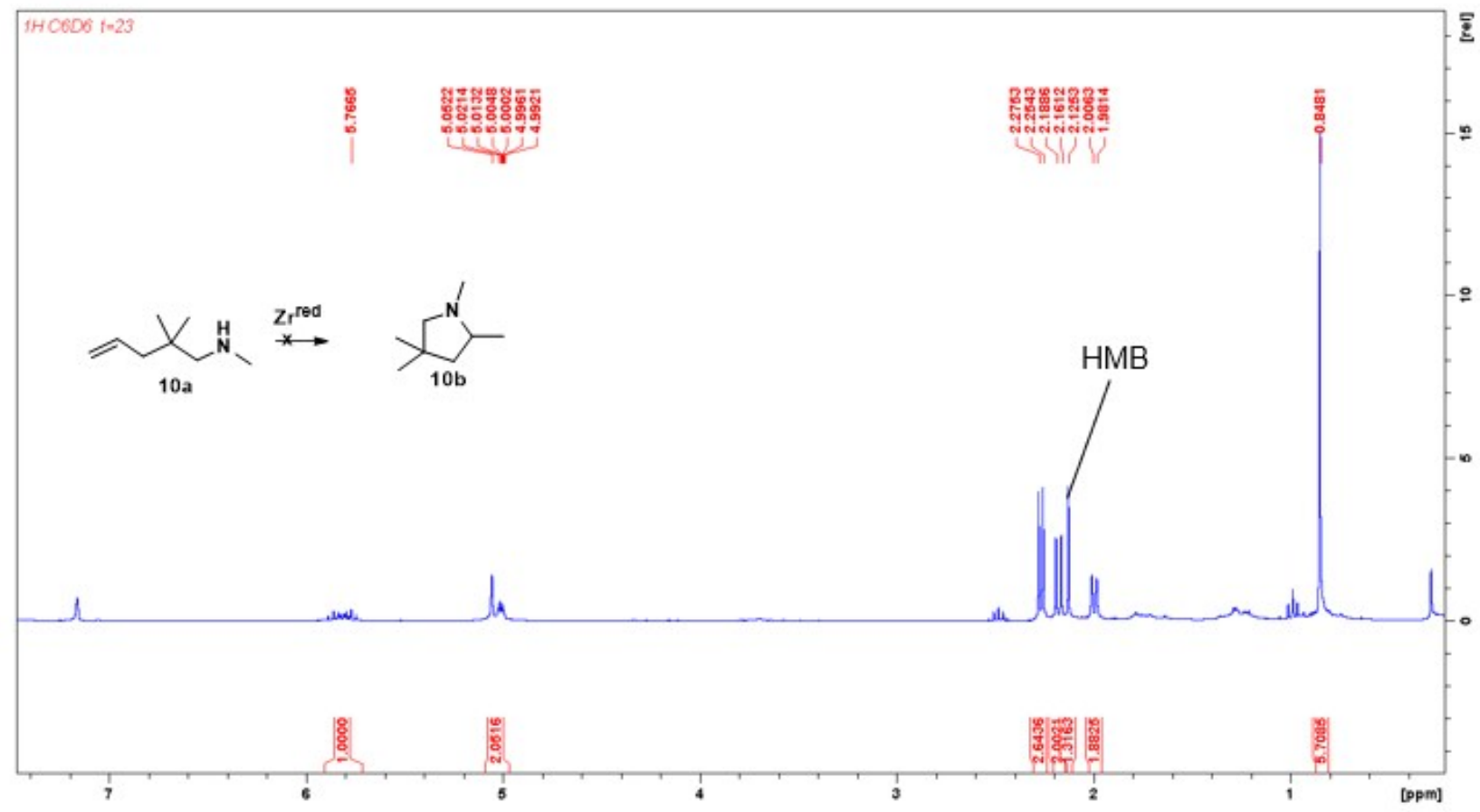

Figure S27: ${ }^{1} \mathrm{H}$ NMR spectrum $\left(\mathrm{C}_{6} \mathrm{D}_{6}, 500 \mathrm{MHz}, 298 \mathrm{~K}\right)$ of $\mathbf{1 0 a}$ catalyzed by (thiolfan*) $\mathrm{Zr}\left(\mathrm{NEt}_{2}\right)_{2}$ (Table 1, entry 19): $\delta 0.84$ (s, 6H, $\left.\mathrm{C}\left(\mathrm{CH}_{3}\right)_{2}\right), 1.98$ (d, 2H, $\left.\mathrm{CHCH}_{2}\right), 2.16\left(\mathrm{~d}, 2 \mathrm{H}, \mathrm{NHCH}_{2}\right), 2.27$ (d, $3 \mathrm{H} \mathrm{NHCH}$ ) $4.99\left(\mathrm{~m}, 2 \mathrm{H}, \mathrm{CH}=\mathrm{CH}_{2}\right) 5.76\left(\mathrm{~m}, 1 \mathrm{H}, \mathrm{CH}=\mathrm{CH}_{2}\right)$.

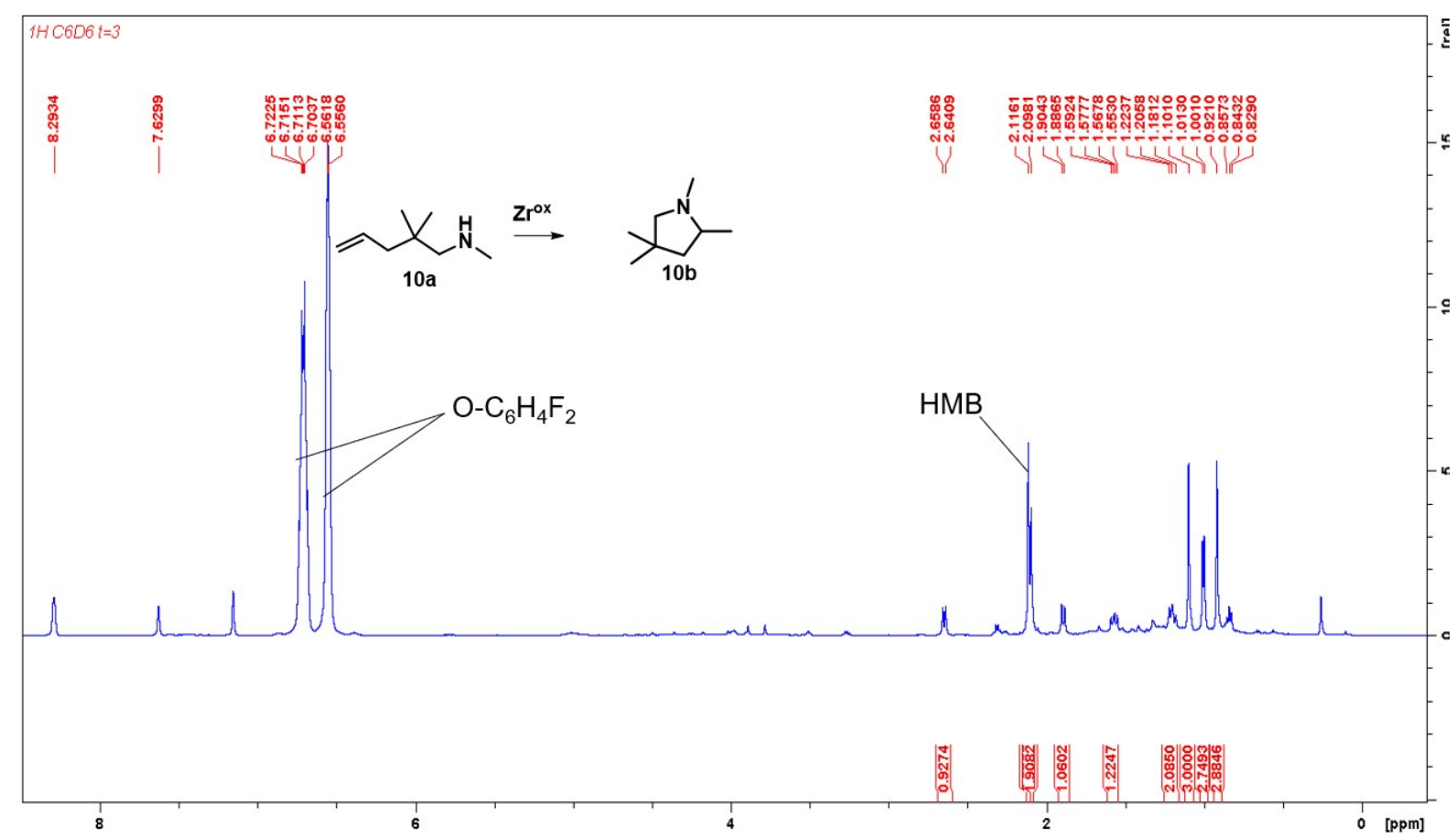

Figure S28: ${ }^{1} \mathrm{H}$ NMR spectrum $\left(\mathrm{C}_{6} \mathrm{D}_{6}, 500 \mathrm{MHz}, 298 \mathrm{~K}\right)$ of $\mathbf{1 0 a}$ catalyzed by the oxidized species (Table 1, entry 20): $\delta 0.81\left(\mathrm{~s}, 3 \mathrm{H}, \mathrm{C}\left(\mathrm{CH}_{3}\right)_{2}\right), 1.01\left(\mathrm{~d}, 3 \mathrm{H}, \mathrm{CHCH}_{2}\right), 1.10\left(\mathrm{~s}, 3 \mathrm{H}, \mathrm{C}\left(\mathrm{CH}_{3}\right)_{2}\right), 1.22$ (dd, 2H, $\mathrm{CHCH}_{2}$ ), 1.59 (q, 1H, MeCH), 2.09 (s, 3H, $\mathrm{NCH}_{3}$ ), 2.65, 1.91 (d, 2H, $\mathrm{MeNCH}_{2}$ ). 


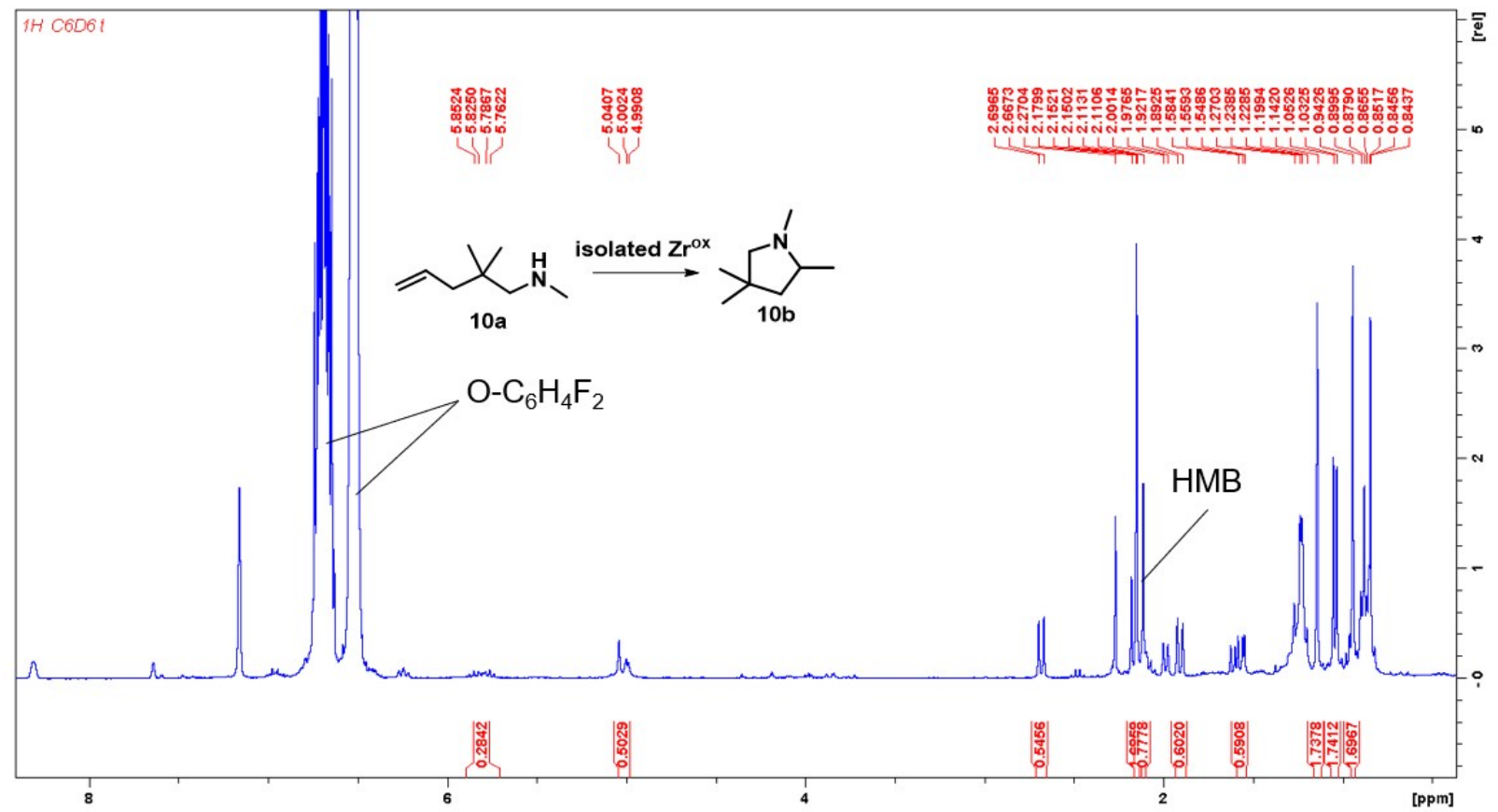

Figure S29: ${ }^{1} \mathrm{H}$ NMR spectrum $\left(\mathrm{C}_{6} \mathrm{D}_{6}, 300 \mathrm{MHz}, 298 \mathrm{~K}\right)$ of $\mathbf{1 0 a}$ catalyzed by the isolated oxidized species $\left(56 \%\right.$ conversion after $105 \mathrm{~h}$ in 1: $4 o$-DFB: $\left.\mathrm{C}_{6} \mathrm{D}_{6}\right): \delta 0.94\left(\mathrm{~s}, 3 \mathrm{H}, \mathrm{C}\left(\mathrm{CH}_{3}\right)_{2}\right), 1.05(\mathrm{~d}, 3 \mathrm{H}$, $\left.\mathrm{CHCH}_{2}\right), 1.14$ (s, 3H, C(CH $\left.)_{2}\right), 1.22$ (m, 2H, $\left.\mathrm{MeCHCH}_{2}\right), 1.59$ (q, 1H, MeCH), 1.90, 2.69 (d, 2H, $\left.\mathrm{MeNCH}_{2}\right), 2.09\left(\mathrm{~s}, 3 \mathrm{H}, \mathrm{NCH}_{3}\right)$. The peaks at 5.85, 5.00, 2.27, 2.17, 2.00, 1.23, $0.85 \mathrm{ppm}$ are attributed to residual $\mathbf{1 0 a}$.

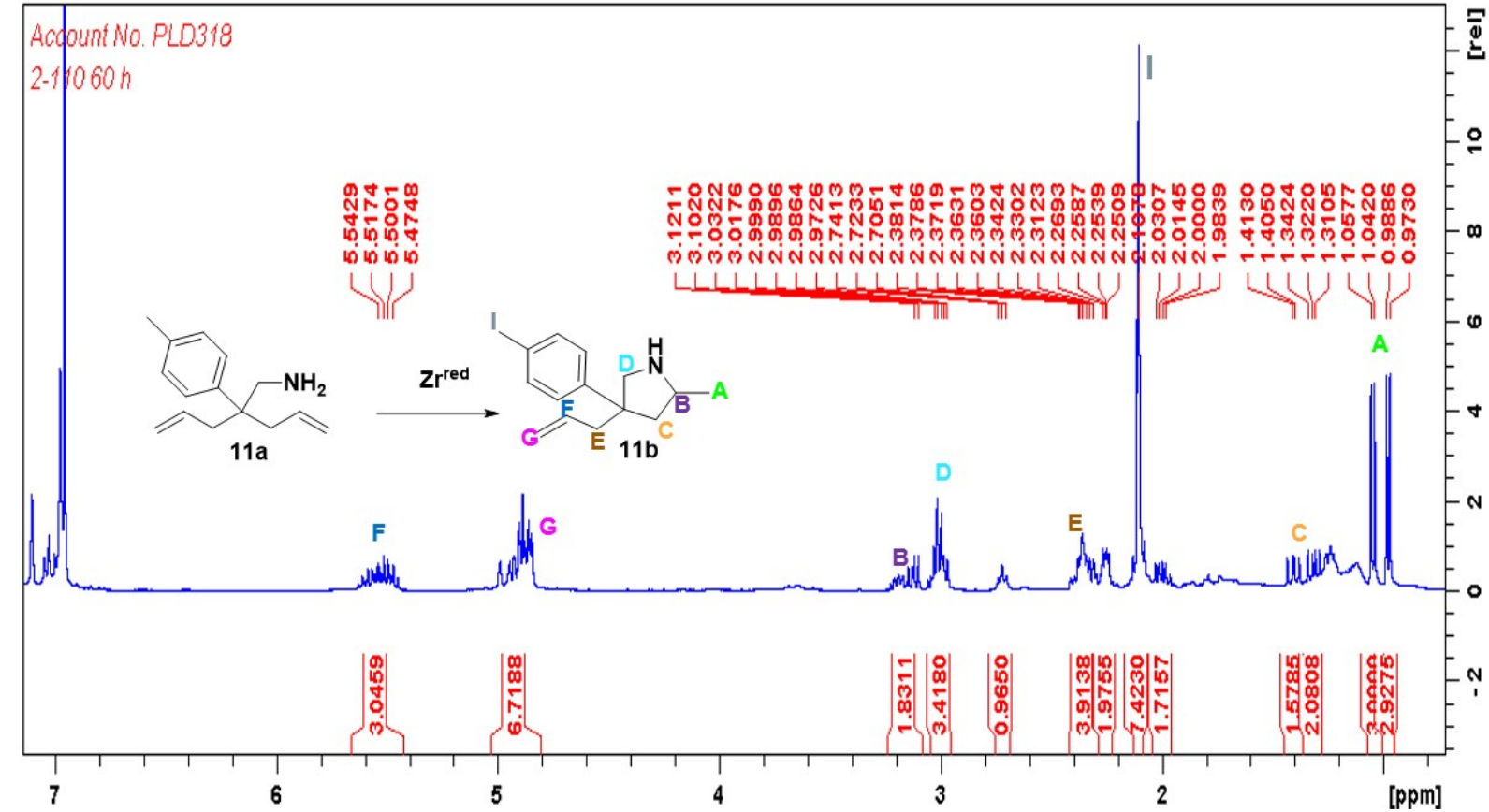

Figure S30: ${ }^{1} \mathrm{H}$ NMR spectrum $\left(\mathrm{C}_{6} \mathrm{D}_{6}, 300 \mathrm{MHz}, 298 \mathrm{~K}\right)$ of 11a (1:1 isomers) catalyzed by (thiolfan*)Zr( $\left.\mathrm{NEt}_{2}\right)_{2}$ (Table 1, entry 21): $\delta 0.97\left(\mathrm{~d}, 3 \mathrm{H}, \mathrm{CHCH}_{3}\right), 1.05$ (d, 3H, $\left.\mathrm{CHCH}_{3}\right), 1.32$ (dd, $\left.2 \mathrm{H}, \mathrm{CHCH}_{2}\right), 1.41\left(\mathrm{dd}, 2 \mathrm{H}, \mathrm{CHCH}_{2}\right), 2.10\left(\mathrm{~s}, 6 \mathrm{H}, \mathrm{PhCH}_{3}\right) 2.47\left(\mathrm{~m}, 4 \mathrm{H}, \mathrm{CCH}_{2}\right), 3.01(\mathrm{~m}, 4 \mathrm{H}$, $\left.\mathrm{NHCH}_{2}\right), 3.22(\mathrm{~m}, 2 \mathrm{H}, \mathrm{NHCHMe}), 5.07\left(\mathrm{~m}, 4 \mathrm{H}, \mathrm{CH}=\mathrm{CH}_{2}\right), 5.69\left(\mathrm{~m}, 2 \mathrm{H}, \mathrm{CH}=\mathrm{CH}_{2}\right)$. The peak at $2.93 \mathrm{ppm}$ is attributed to residual 11a. 


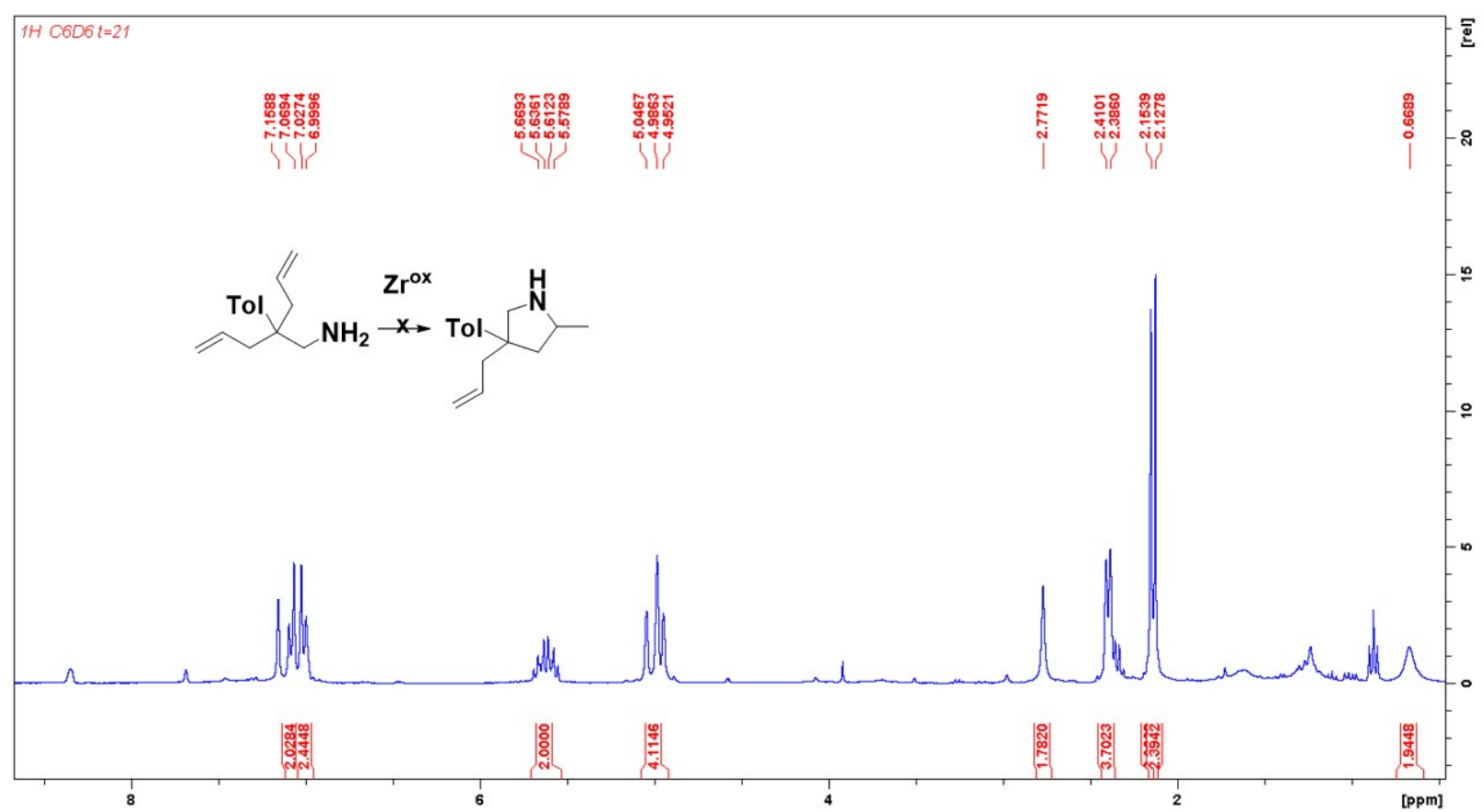

Figure S31: ${ }^{1} \mathrm{H}$ NMR spectrum $\left(\mathrm{C}_{6} \mathrm{D}_{6}, 300 \mathrm{MHz}, 298 \mathrm{~K}\right)$ of 11a $(1: 1$ isomers $)$ catalyzed by the oxidized species (Table 1, entry 22): $\delta 0.66\left(\mathrm{br}, 2 \mathrm{H}, \mathrm{NH} \mathrm{H}_{2}\right), 2.15\left(\mathrm{~s}, 2 \mathrm{H}, \mathrm{PhCH}_{3}\right), 2.41(\mathrm{~d}, 4 \mathrm{H}$, $\left.\mathrm{CH}_{2}=\mathrm{CHCH}_{2}\right), 2.77\left(\mathrm{~s}, 2 \mathrm{H}, \mathrm{NH}_{2} \mathrm{CH}_{2}\right), 5.04\left(\mathrm{~m}, 4 \mathrm{H}, \mathrm{CH}=\mathrm{CH}_{2}\right), 5.66\left(\mathrm{~m}, 2 \mathrm{H}, \mathrm{CH}=\mathrm{CH}_{2}\right)$. The peak at $2.13 \mathrm{ppm}$ is attributed to $\mathrm{HMB}$.

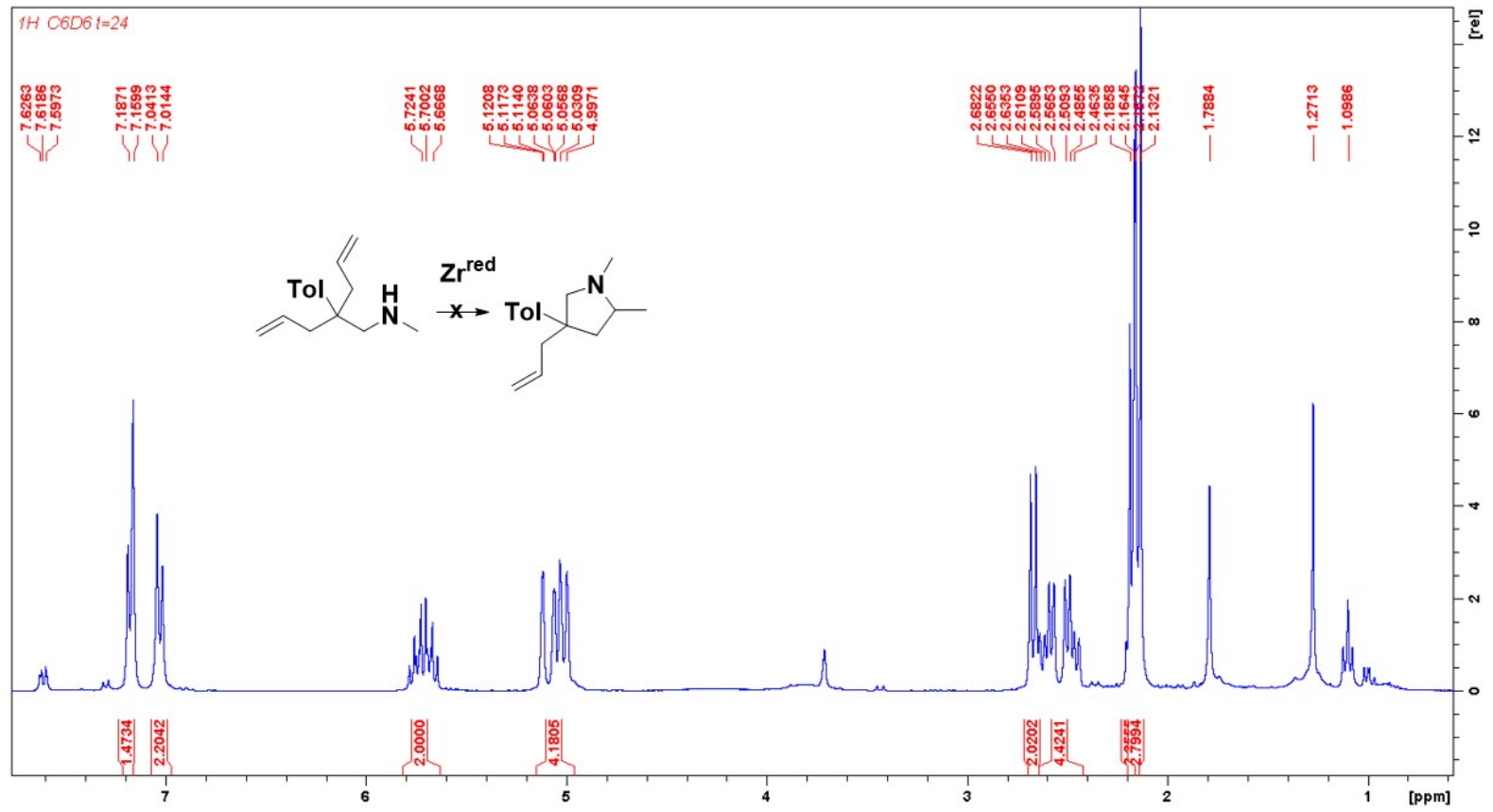

Figure S32: ${ }^{1} \mathrm{H}$ NMR spectrum $\left(\mathrm{C}_{6} \mathrm{D}_{6}, 300 \mathrm{MHz}, 298 \mathrm{~K}\right)$ of 12a (1:1 isomers) catalyzed by (thiolfan*) $\mathrm{Zr}\left(\mathrm{NEt}_{2}\right)_{2}$ (Table 1, entry 23): $\delta 2.16\left(\mathrm{~s}, 3 \mathrm{H}, \mathrm{NHCH}_{3}\right), 2.18\left(\mathrm{~s}, 3 \mathrm{H}, \mathrm{PhCH}_{3}\right), 2.50-2.61$ (ddd, $\left.4 \mathrm{H}, \mathrm{CH}_{2}=\mathrm{CHCH}_{2}\right), 2.65\left(\mathrm{~d}, 2 \mathrm{H}, \mathrm{NHCH}_{2}\right), 5.04\left(\mathrm{~m}, 4 \mathrm{H}, \mathrm{CH}_{2}=\mathrm{CH}\right), 5.65\left(\mathrm{~m}, 2 \mathrm{H}, \mathrm{CH}_{2}=\mathrm{CH}\right)$, $7.13(\mathrm{~d}, 2 \mathrm{H}, \operatorname{ArH}), 7.23(\mathrm{~d}, 2 \mathrm{H}, \operatorname{ArH})$. The peak at $2.12 \mathrm{ppm}$ is attributed to HMB. The peaks at $1.27,1.78$, and $7.62 \mathrm{ppm}$ are attributed to (thiolfan*) $\mathrm{Zr}\left(\mathrm{NEt}_{2}\right)_{2}$. 


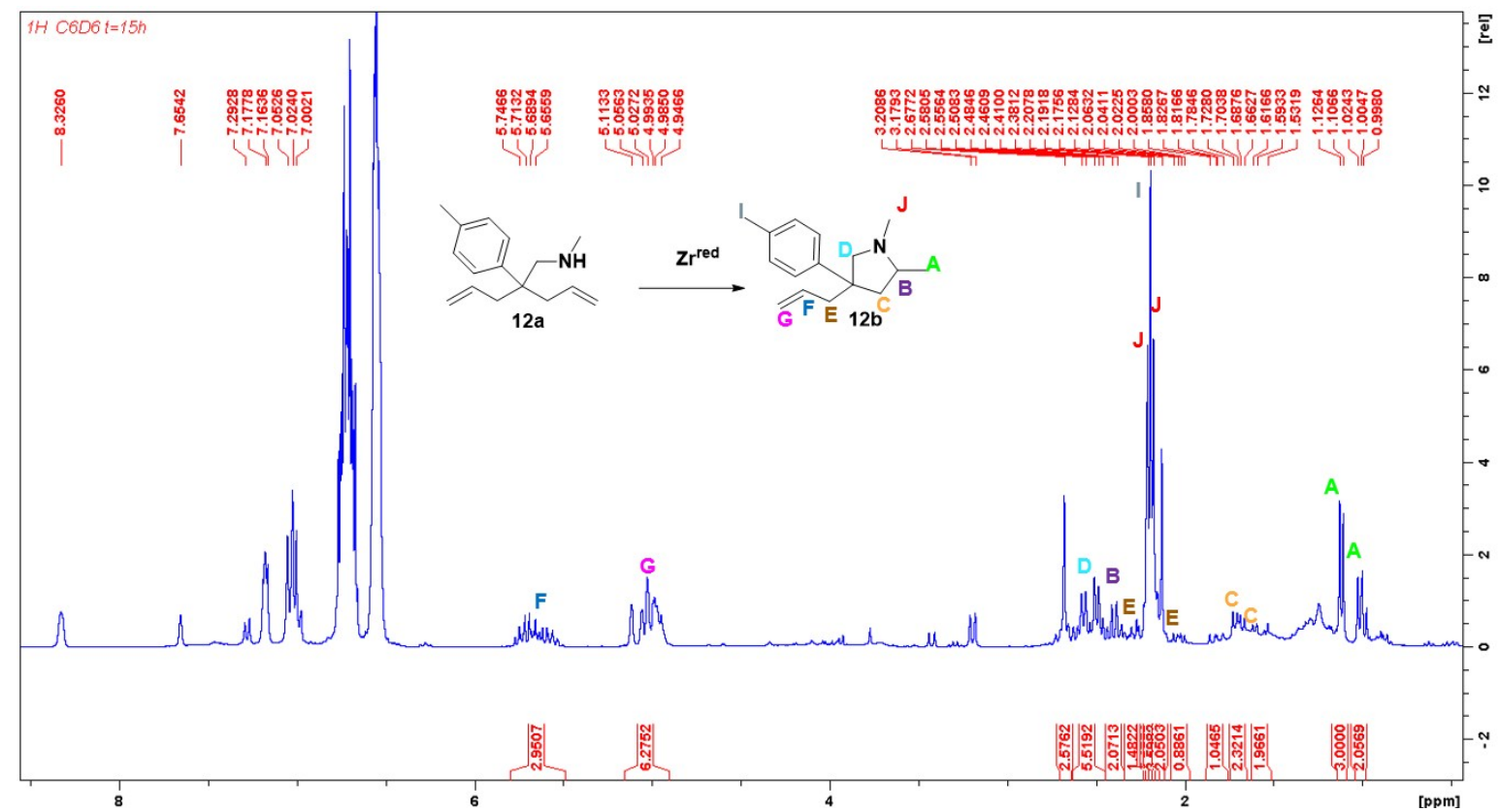

Figure S33: ${ }^{1} \mathrm{H}$ NMR spectrum $\left(\mathrm{C}_{6} \mathrm{D}_{6}, 300 \mathrm{MHz}, 298 \mathrm{~K}\right)$ of 12a (3:2 isomers) catalyzed by the oxidized species (Table 1, entry 24): $\delta 0.97\left(\mathrm{~d}, 3 \mathrm{H}, \mathrm{CHCH}_{3}\right), 1.10\left(\mathrm{~d}, 2 \mathrm{H}, \mathrm{CHCH}_{3}\right), 1.32(\mathrm{dd}, 2 \mathrm{H}$, $\left.\mathrm{CHCH}_{2}\right), 1.61\left(\mathrm{dd}, 2 \mathrm{H}, \mathrm{CHCH}_{2}\right), 1.72\left(\mathrm{dd}, 2 \mathrm{H}, \mathrm{CHCH}_{2}\right), 1.85,2.06,2.66\left(\mathrm{~m}, 4 \mathrm{H}, \mathrm{CH}_{2}=\mathrm{CHCH}_{2}\right)$, 2.19 (s, 5H, $\left.\mathrm{PhCH}_{3}\right), 2.17,2.20$ (s, 5H NCH $\left.\mathrm{H}_{3}\right), 2.41$ (m, 2H, $\left.\mathrm{CH}_{3} \mathrm{NCH}\right), 2.46-2.58\left(\mathrm{~m}, 4 \mathrm{H}, \mathrm{CCH}_{2}\right)$, $5.05\left(\mathrm{~m}, 6 \mathrm{H}, \mathrm{CH}=\mathrm{CH}_{2}\right), 5.69\left(\mathrm{~m}, 3 \mathrm{H}, \mathrm{CH}=\mathrm{CH}_{2}\right)$. The peak at $2.67 \mathrm{ppm}$ is attributed to residual 12a. The peak at 2.12 is attributed to HMB. 


\section{Control experiments:}

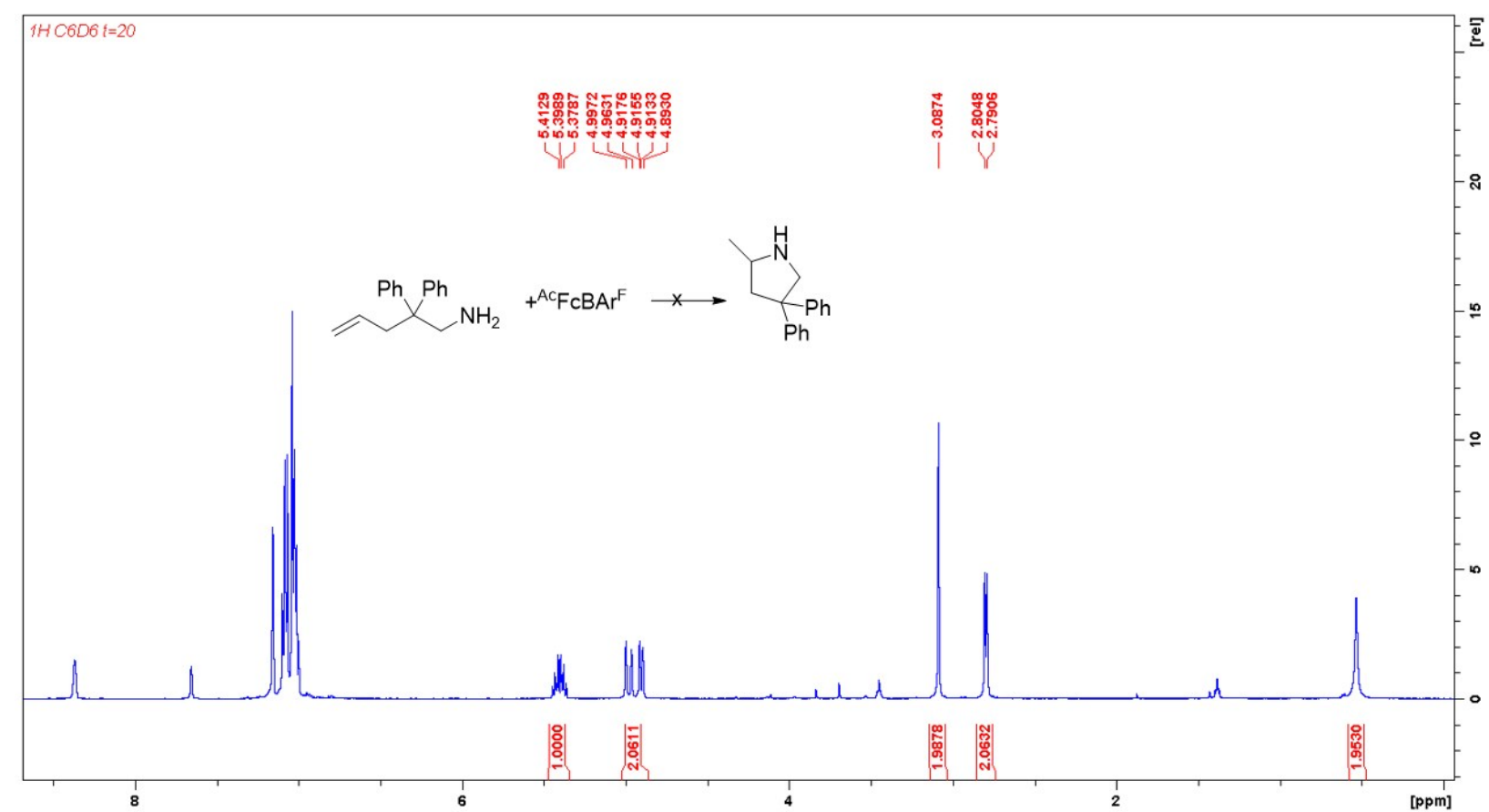

Figure S34: ${ }^{1} \mathrm{H}$ NMR spectrum $\left(\mathrm{C}_{6} \mathrm{D}_{6}, 300 \mathrm{MHz}, 298 \mathrm{~K}\right)$ of $1 \mathrm{a}$ and ${ }^{\mathrm{Ac}} \mathrm{FcBAr}{ }^{\mathrm{F}}: \delta 0.49(\mathrm{~s}, 2 \mathrm{H} \mathrm{NH})_{2}$, $2.88\left(\mathrm{~d}, 2 \mathrm{H}, \mathrm{CHCH}_{2}\right), 3.16\left(\mathrm{~s}, 2 \mathrm{H}, \mathrm{NH}_{2} \mathrm{CH}_{2}\right), 5.02\left(\mathrm{~m}, 2 \mathrm{H}, \mathrm{CH}_{2}=\mathrm{CH}\right), 5.45\left(\mathrm{~m}, 1 \mathrm{H}, \mathrm{CH}_{2}=\mathrm{CH}\right) 7.08$ $(\mathrm{m}, 10 \mathrm{H} \mathrm{ArH})$.

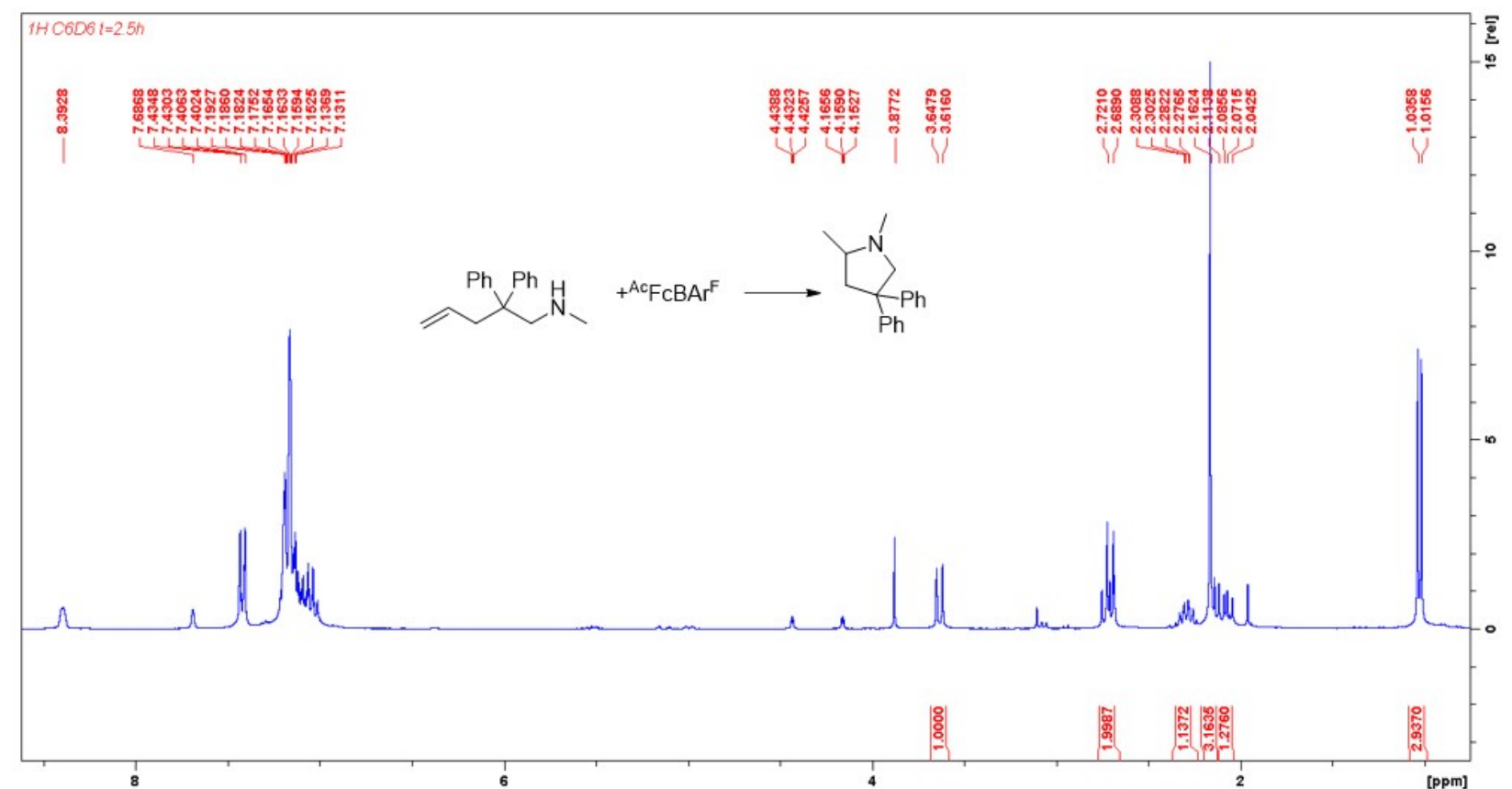

Figure S35: ${ }^{1} \mathrm{H}$ NMR spectrum $\left(\mathrm{C}_{6} \mathrm{D}_{6}, 500 \mathrm{MHz}, 298 \mathrm{~K}\right)$ of $\mathbf{2 a}$ and ${ }^{A c} \mathrm{FcBAr}{ }^{\mathrm{F}}: \delta 1.03(\mathrm{~d}, 3 \mathrm{H}$, $\left.\mathrm{NCH}_{3}\right), 2.04$ (t, $\left.1 \mathrm{H}, \mathrm{MeCHCH}_{2}\right), 2.08\left(\mathrm{~s}, 3 \mathrm{H}, \mathrm{NCHCH}_{3}\right), 2.28\left(\mathrm{~m}, 1 \mathrm{H}, \mathrm{MeCHCH}_{2}\right), 2.71(\mathrm{~m}, 2 \mathrm{H}$, $\left.\mathrm{NMeCH}_{2}\right), 3.65\left(\mathrm{~d}, 1 \mathrm{H}, \mathrm{MeCHCH}_{2}\right)$. 


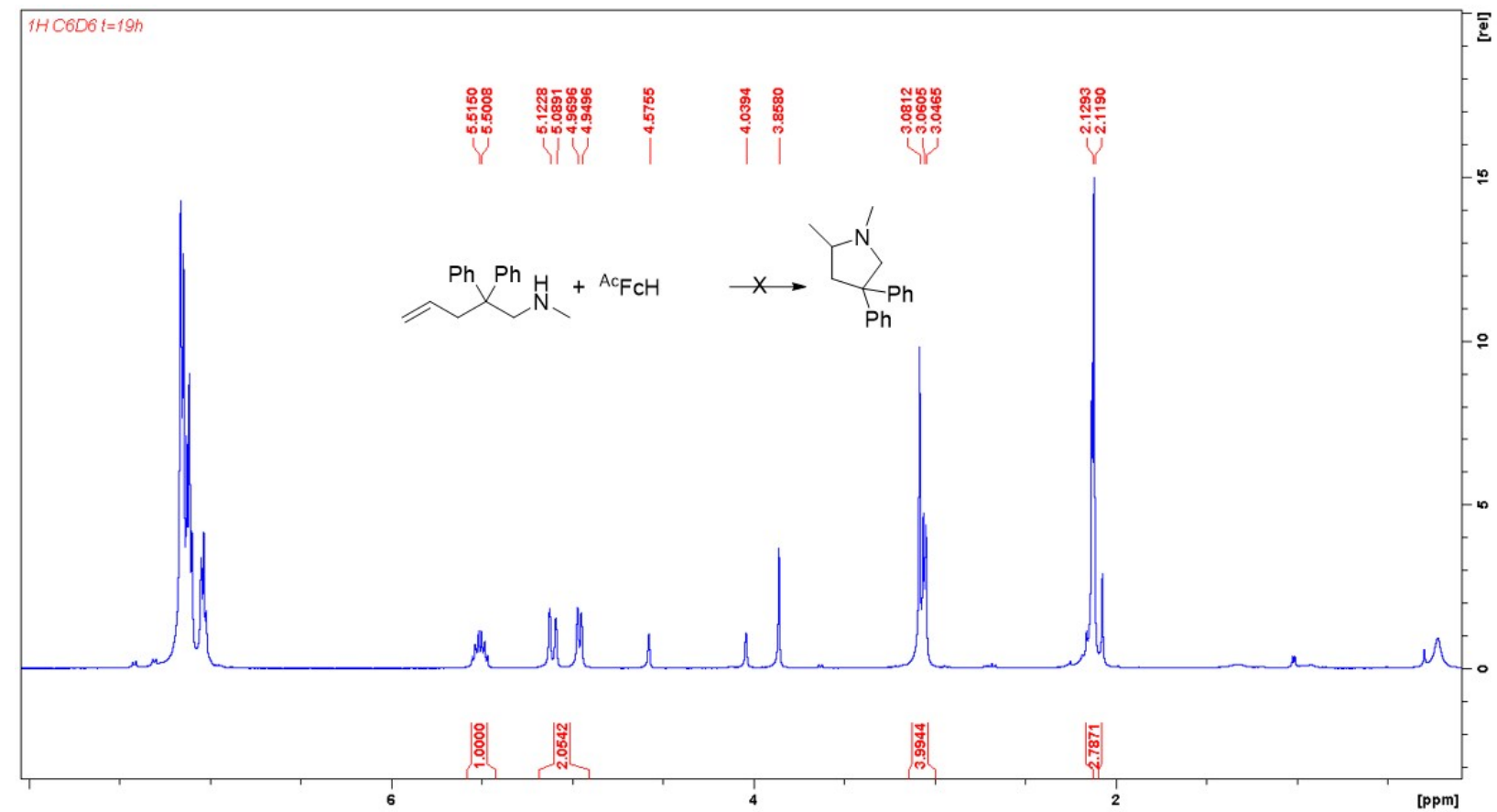

Figure S36: ${ }^{1} \mathrm{H}$ NMR spectrum $\left(\mathrm{C}_{6} \mathrm{D}_{6}, 500 \mathrm{MHz}, 298 \mathrm{~K}\right)$ of $\mathbf{2 a}$ and by acetylferrocene: $\delta 2.11(\mathrm{~s}$, $\left.3 \mathrm{H}, \mathrm{NHCH}_{3}\right), 3.04\left(\mathrm{~d}, 2 \mathrm{H}, \mathrm{CHCH}_{2}\right), 3.09\left(\mathrm{~s}, 2 \mathrm{H}, \mathrm{NHCH}_{2}\right), 5.12\left(\mathrm{~m}, 2 \mathrm{H}, \mathrm{CH}_{2}=\mathrm{CH}\right), 5.51(\mathrm{~m}, 1 \mathrm{H}$, $\mathrm{CH}_{2}=\mathrm{CH}$ ). The peak at $2.12 \mathrm{ppm}$ is attributed to HMB.

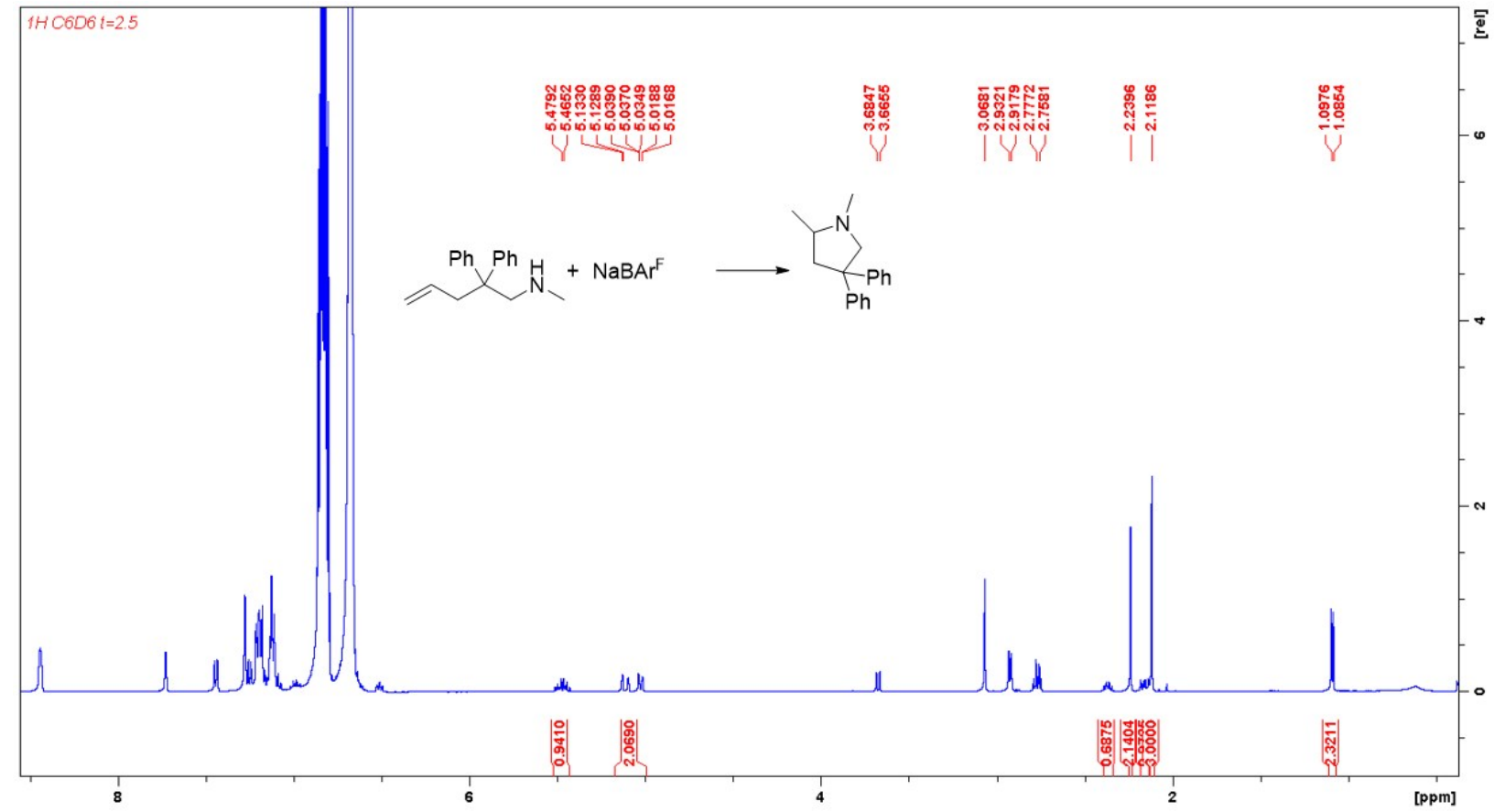

Figure S37: ${ }^{1} \mathrm{H}$ NMR spectrum $\left(o-\mathrm{C}_{6} \mathrm{H}_{4} \mathrm{~F}_{2}: \mathrm{C}_{6} \mathrm{D}_{6} 1: 4,500 \mathrm{MHz}, 298 \mathrm{~K}\right)$ of $\mathbf{2 a}$ and $\mathrm{NaBAr}{ }^{\mathrm{F}}: \delta 0.97$ (d, $\left.\mathrm{NCH}_{3}\right), 2.04$ (t, $\left.\mathrm{MeCHCH}_{2}\right), 1.99\left(\mathrm{~s}, \mathrm{NCHCH}_{3}\right), 2.28\left(\mathrm{~m}, \mathrm{MeCHCH}_{2}\right), 2.71$ (m, $\mathrm{NMeCH}_{2}$ ), $3.65\left(\mathrm{~d}, \mathrm{MeCHCH}_{2}\right)$. The peak at $2.12 \mathrm{ppm}$ is attributed to HMB. 


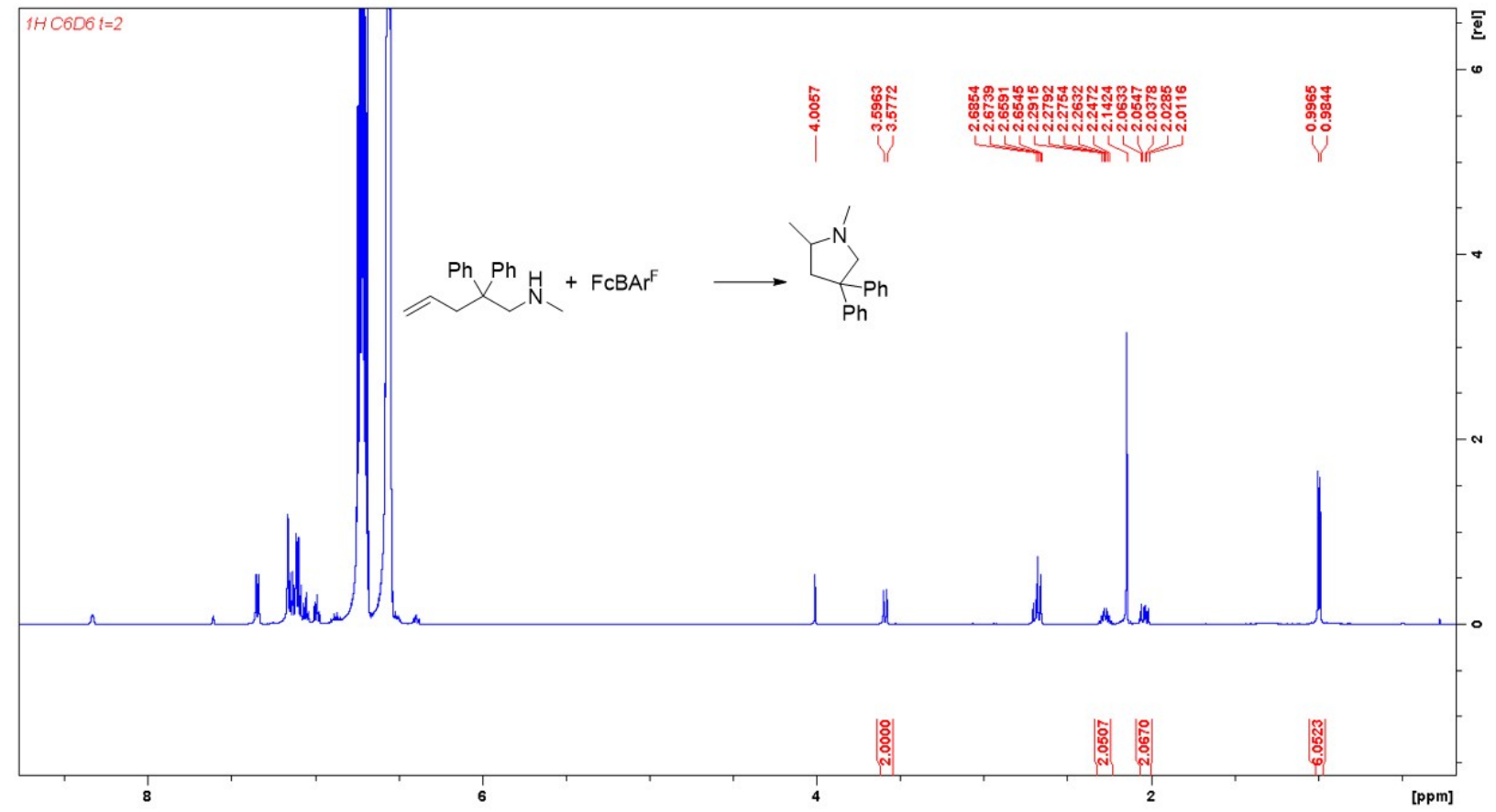

Figure S38: ${ }^{1} \mathrm{H}$ NMR spectrum $\left(o-\mathrm{C}_{6} \mathrm{H}_{4} \mathrm{~F}_{2}: \mathrm{C}_{6} \mathrm{D}_{6} 1: 4,500 \mathrm{MHz}, 298 \mathrm{~K}\right)$ of $2 \mathbf{a}$ and $\mathrm{FcBAr}{ }^{\mathrm{F}}: \delta 1.03$ (d, $\left.3 \mathrm{H}, \mathrm{NCH}_{3}\right), 2.04$ (t, 1H, $\left.\mathrm{MeCHCH}_{2}\right), 2.08$ (s, 3H, $\left.\mathrm{NCHCH}_{3}\right), 2.28\left(\mathrm{~m}, 1 \mathrm{H}, \mathrm{MeCHCH}_{2}\right), 2.71$ $\left(\mathrm{m}, 2 \mathrm{H}, \mathrm{NMeCH} \mathrm{H}_{2}\right), 3.65\left(\mathrm{~d}, 1 \mathrm{H}, \mathrm{MeCHCH}_{2}\right)$. The peak at $2.14 \mathrm{ppm}$ is attributed to $\mathrm{HMB}$, and the peak at $4.0 \mathrm{ppm}$ is attributed to ferrocene.

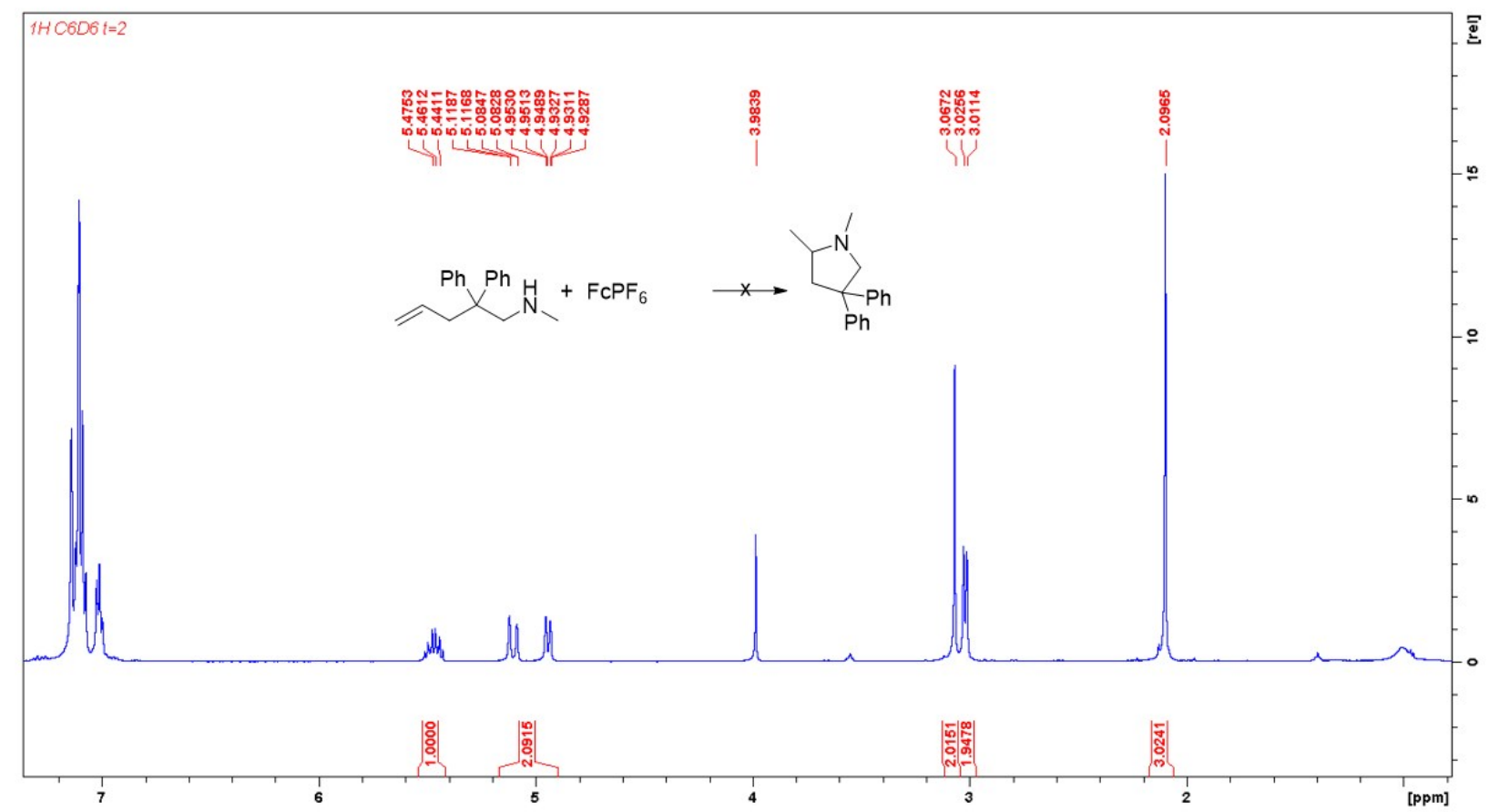

Figure S39: ${ }^{1} \mathrm{H}$ NMR spectrum $\left(\mathrm{C}_{6} \mathrm{D}_{6}, 500 \mathrm{MHz}, 298 \mathrm{~K}\right)$ of $\mathbf{2 a}$ and $\mathrm{FcPF}_{6}: \delta 2.08\left(\mathrm{~s}, 3 \mathrm{H}, \mathrm{NHCH}_{3}\right)$, $3.01\left(\mathrm{~d}, 2 \mathrm{H}, \mathrm{CHCH}_{2}\right), 3.06\left(\mathrm{~s}, 2 \mathrm{H}, \mathrm{NHCH}_{2}\right), 5.11\left(\mathrm{dd}, 2 \mathrm{H}, \mathrm{CH}_{2}=\mathrm{CH}\right), 5.47\left(\mathrm{~m}, 1 \mathrm{H}, \mathrm{CH}_{2}=\mathrm{CH}\right)$. The peak at $3.98 \mathrm{ppm}$ is attributed to ferrocene. 


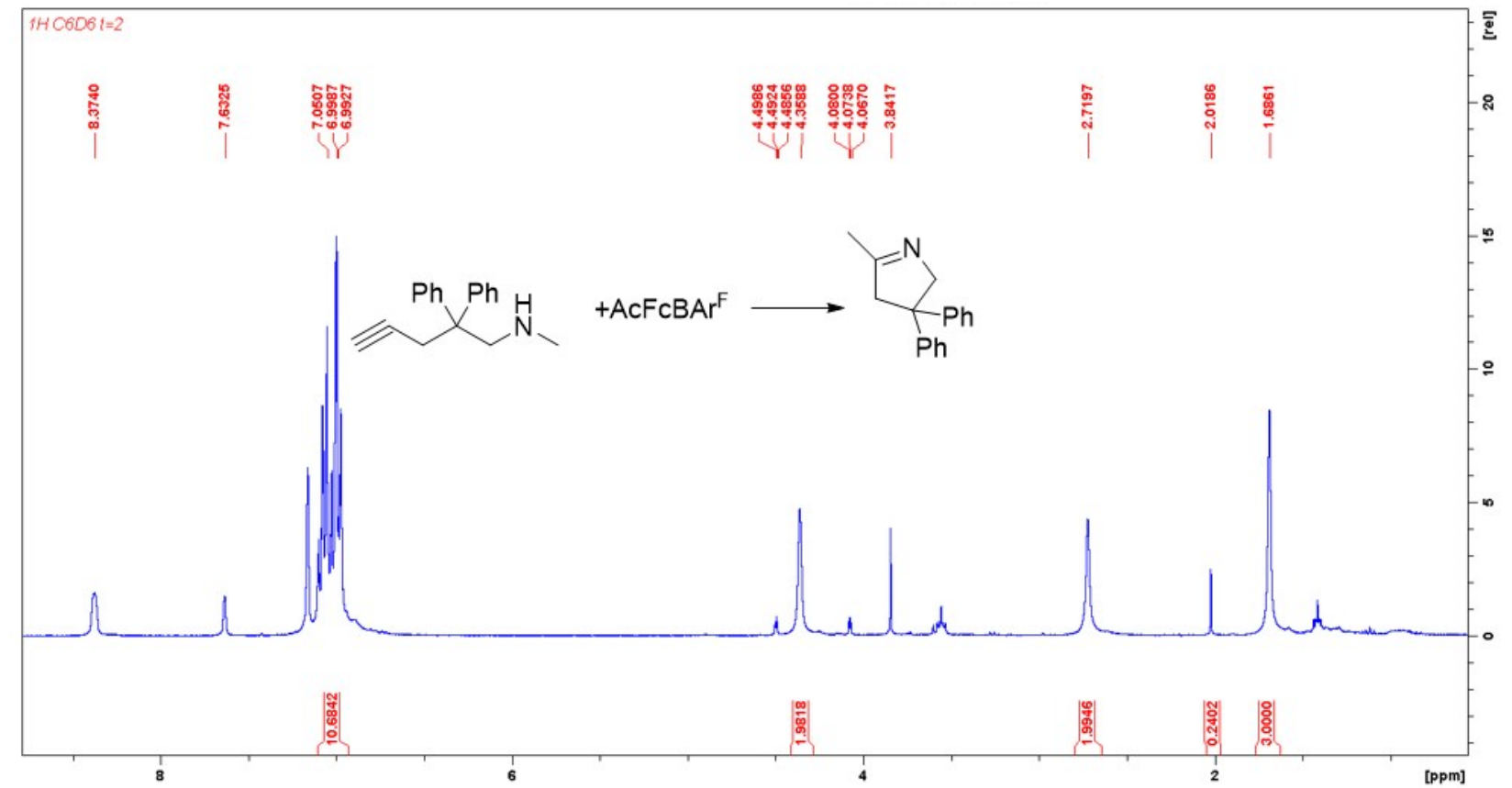

Figure S40: ${ }^{1} \mathrm{H}$ NMR spectrum $\left(\mathrm{C}_{6} \mathrm{D}_{6}, 500 \mathrm{MHz}, 298 \mathrm{~K}\right)$ of $7 \mathbf{a}$ and ${ }^{A c F c B A r}{ }^{\mathrm{F}}: \delta 1.68(\mathrm{~s}, 3 \mathrm{H}$, $\left.\mathrm{CCH}_{3}\right), 2.71\left(\mathrm{~s}, 2 \mathrm{H}, \mathrm{CCH}_{2}\right), 4.48\left(\mathrm{~s}, 2 \mathrm{H}, \mathrm{NCH}_{2}\right), 7.00(\mathrm{~m}, 10 \mathrm{H}, \mathrm{ArH})$.

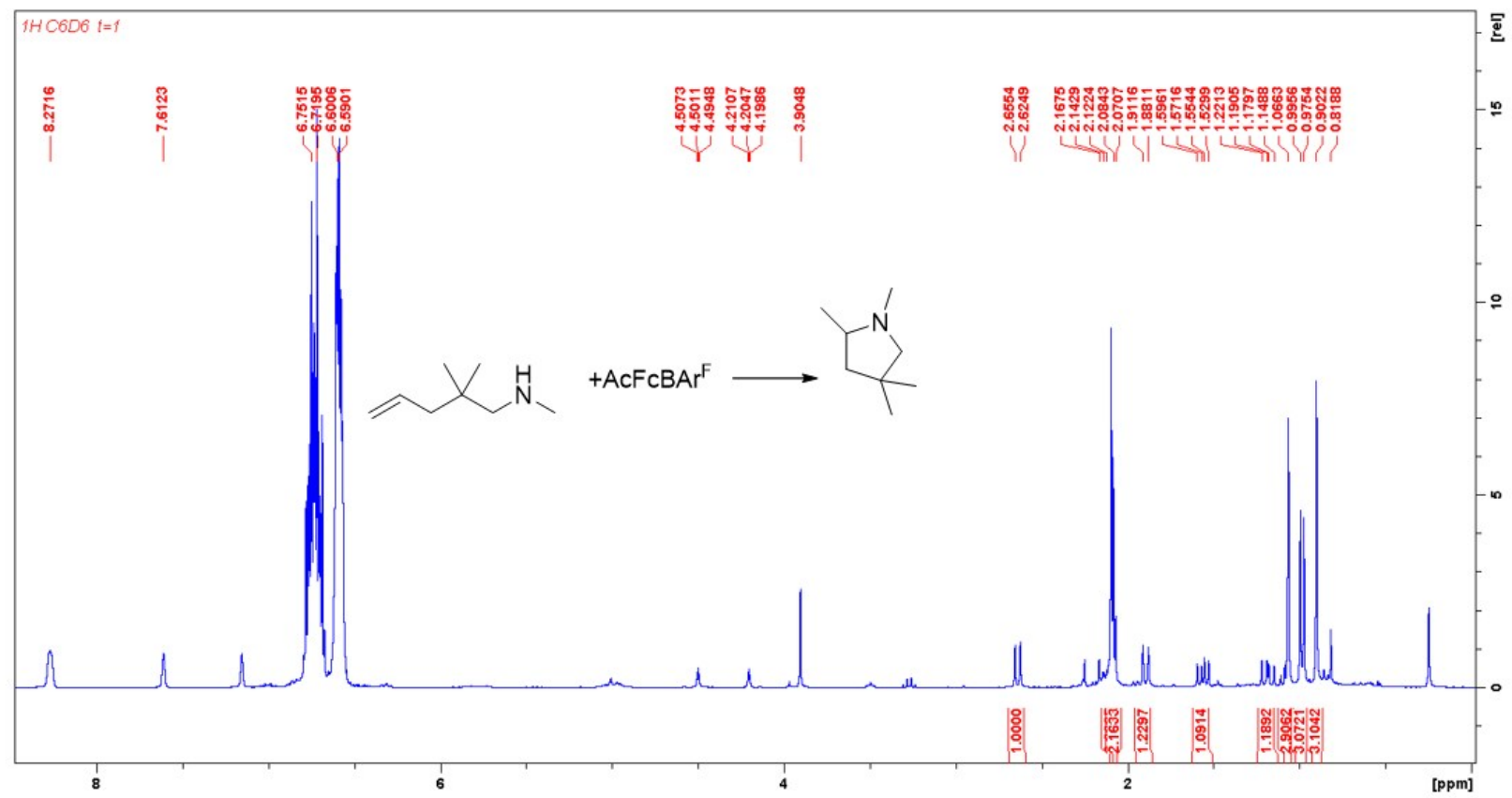

Figure S41: ${ }^{1} \mathrm{H}$ NMR spectrum $\left(\mathrm{C}_{6} \mathrm{D}_{6}, 500 \mathrm{MHz}, 298 \mathrm{~K}\right)$ of $\mathbf{1 0 a}$ catalyzed by ${ }^{\mathrm{Ac} F c B A r}{ }^{\mathrm{F}}: \delta 0.81(\mathrm{~s}$, $\left.3 \mathrm{H}, \mathrm{C}\left(\mathrm{CH}_{3}\right)_{2}\right), 1.01\left(\mathrm{~d}, 3 \mathrm{H}, \mathrm{CHCH}_{2}\right), 1.10\left(\mathrm{~s}, 3 \mathrm{H}, \mathrm{C}\left(\mathrm{CH}_{3}\right)_{2}\right), 1.22\left(\mathrm{~m}, 2 \mathrm{H}, \mathrm{MeCHCH}_{2}\right), 1.59(\mathrm{~m}$, $\left.2 \mathrm{H}, \mathrm{MeNCH}_{2}\right) 2.09\left(\mathrm{~s}, 1 \mathrm{H}, \mathrm{NCH}_{3}\right), 2.65(\mathrm{~d}, 2 \mathrm{H}, \mathrm{MeCH})$. The peaks at $6.76 \mathrm{ppm}$ are attributed to residual $o-\mathrm{C}_{6} \mathrm{~F}_{2} \mathrm{H}_{4}$. 


\section{Cyclic voltammetry:}

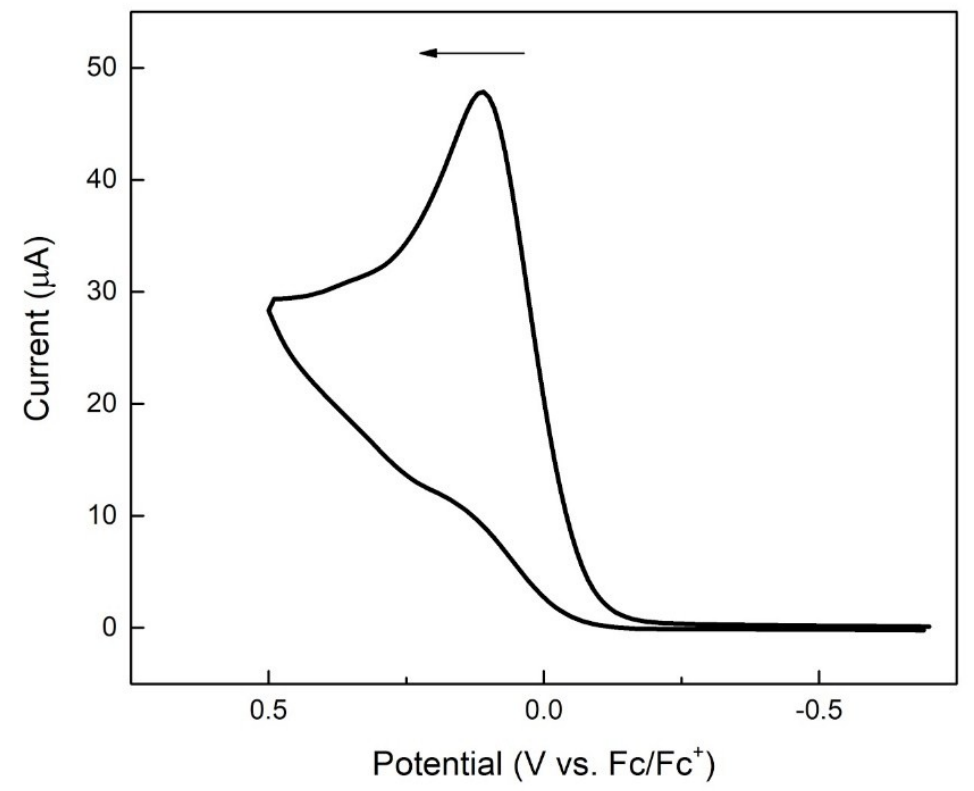

Figure S42: A region of the cyclic voltammetry diagram of (thiolfan*) $\mathrm{Zr}\left(\mathrm{NEt}_{2}\right)_{2}$ recorded in $o$ $\mathrm{F}_{2} \mathrm{C}_{6} \mathrm{H}_{4}$ at $100 \mathrm{mV} \mathrm{s}^{-1}$ with $0.1 \mathrm{M} \mathrm{TPABAr}^{\mathrm{F}}$ as the electrolyte and referenced to the $\mathrm{Fc} / \mathrm{Fc}^{+}$couple. 
Mössbauer spectra:
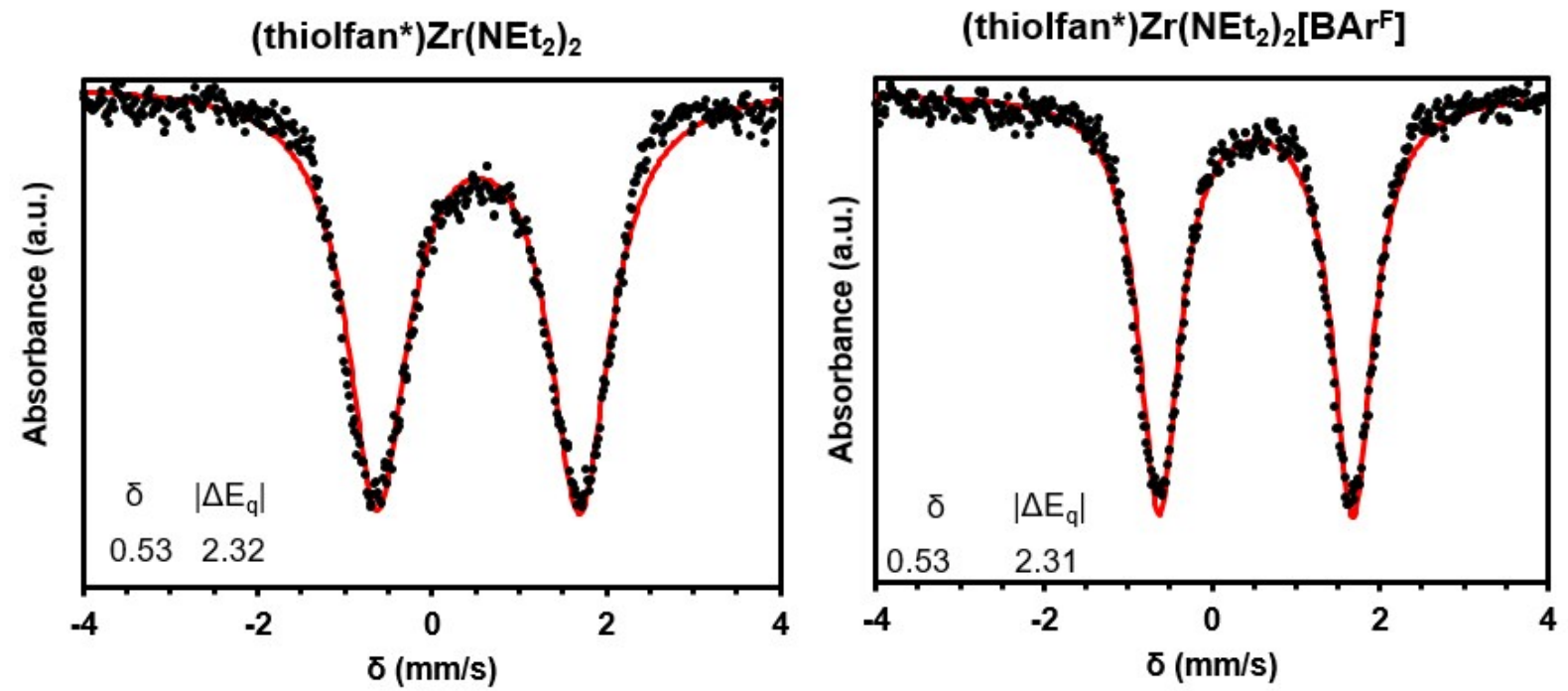

Figure S43: Zero-field ${ }^{57} \mathrm{Fe}$ Mössbauer spectra of (thiolfan*)Zr( $\left(\mathrm{NEt}_{2}\right)_{2}$ (left) and $\left[\left(\right.\right.$ thiolfan $\left.\left.^{*}\right) \mathrm{Zr}\left(\mathrm{NEt}_{2}\right)_{2}\right]\left[\mathrm{BAr}^{\mathrm{F}}\right]$ (right) recorded as solid samples at $80 \mathrm{~K}$. 


\section{EPR spectrum}

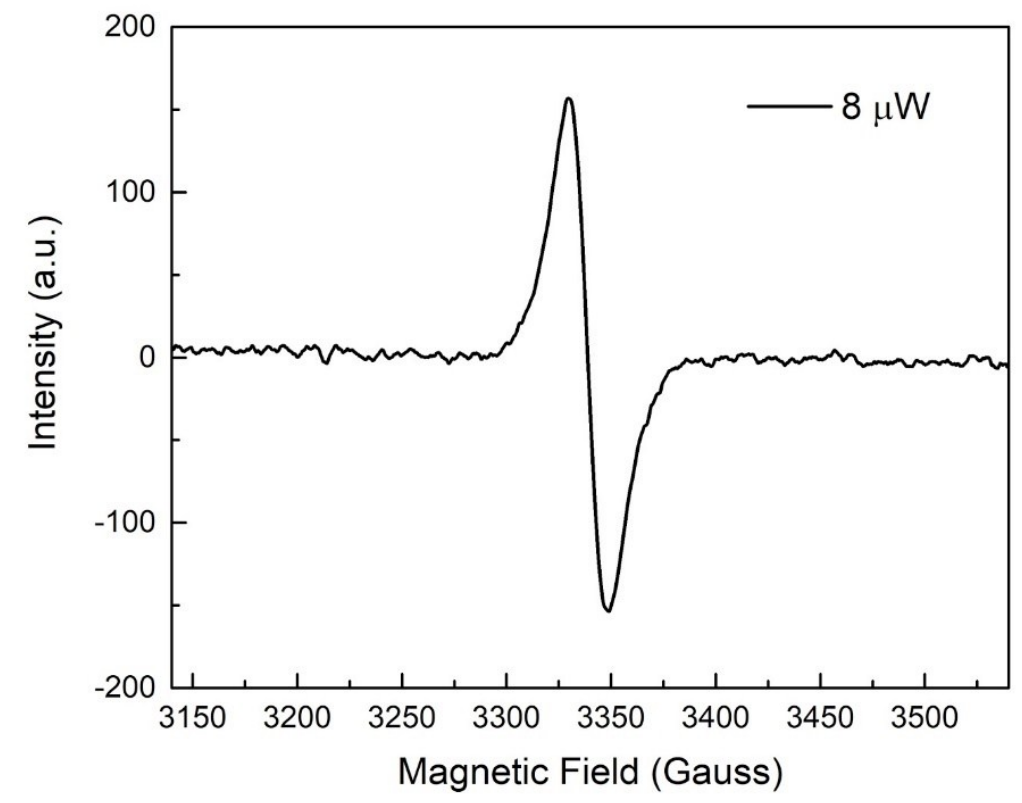

Figure S44: EPR spectrum of [(thiolfan*) $\left.\mathrm{Zr}\left(\mathrm{NEt}_{2}\right)_{2}\right]\left[\mathrm{BAr}{ }^{\mathrm{F}}\right]$ at $77 \mathrm{~K}$ in toluene. Conversion time: 40.96 msec; modulation amplitude: $4.00 \mathrm{G}$; modulation frequency: $100.00 \mathrm{kHz} ; g=2.006$. 


\section{UV-Vis Spectra}

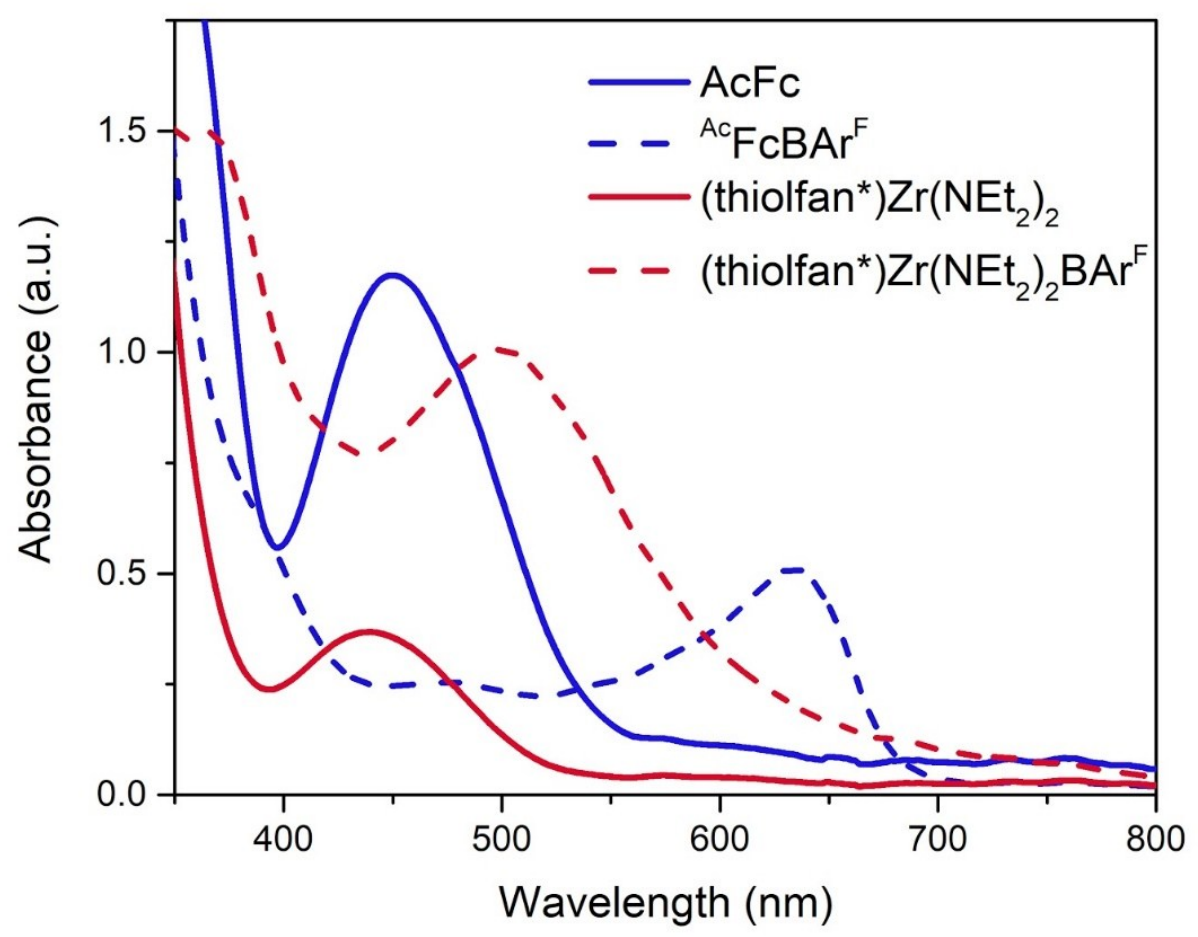

Figure S45: UV-Vis spectra of (thiolfan*) $\mathrm{Zr}\left(\mathrm{NEt}_{2}\right)_{2}$ (red solid line), [(thiolfan*)Zr( $\left.\left.\mathrm{NEt}_{2}\right)_{2}\right]\left[\mathrm{BAr}^{\mathrm{F}}\right]$ (red dash line), acetyl ferrocene (blue solid line), and ${ }^{A c} \mathrm{FcBAr} F$ (blue dash line). 


\section{References}

1. A. B. Pangborn, M. A. Giardello, R. H. Grubbs, R. K. Rosen and F. J. Timmers, Organometallics, 1996, 15, 1518-1520.

2. $\quad$ D. C. Bradley and I. M. Thomas, J. Chem. Soc., 1960, 3857-3861.

3. $\quad$ N. A. Yakelis and R. G. Bergman, Organometallics, 2005, 24, 3579-3581.

4. J. Wei, M. N. Riffel and P. L. Diaconescu, Macromolecules, 2017, 50, 1847-1861.

5. Z. Chai, J. Chu, Y. Qi, M. Tang, J. Hou and G. Yang, RSC Adv., 2017, 7, 1759-1765.

6. P. D. Knight, I. Munslow, P. N. O'Shaughnessy and P. Scott, Chem. Commun., 2004, 894895.

7. T. de Haro and C. Nevado, Adv. Synth. Catal., 2010, 352, 2767-2772.

8. K. Manna, N. Eedugurala and A. D. Sadow, J. Am. Chem. Soc., 2015, 137, 425-435.

9. M. R. Crimmin, M. Arrowsmith, A. G. M. Barrett, I. J. Casely, M. S. Hill and P. A. Procopiou, J. Am. Chem. Soc., 2009, 131, 9670-9685.

10. P. H. Martínez, K. C. Hultzsch and F. Hampel, Chem. Commun., 2006, DOI: 10.1039/B518360J, 2221-2223.

11. L. Hussein, N. Purkait, M. Biyikal, E. Tausch, P. W. Roesky and S. Blechert, Chem. Commun., 2014, 50, 3862-3864.

12. D. Dhar, G. M. Yee, A. D. Spaeth, D. W. Boyce, H. Zhang, B. Dereli, C. J. Cramer and W. B. Tolman, J. Am. Chem. Soc., 2016, 138, 356-368.

13. I. Chávez, A. Alvarez-Carena, E. Molins, A. Roig, W. Maniukiewicz, A. Arancibia, V. Arancibia, H. Brand and J. Manuel Manriquez, J. Organomet. Chem., 2000, 601, 126-132. 